\title{
Fluorescence Phenomena in Amyloid and Amyloidogenic Bionanostructures
}

\author{
B. Apter ${ }^{1}$, N. Lapshina ${ }^{2}$, H. Barhom ${ }^{2}$, B. Fainberg ${ }^{3}$, A. Handelman ${ }^{1}{ }^{1}$, A. Accardo ${ }^{4}$, \\ C. Diaferia ${ }^{4}$ (D) P. Ginzburg ${ }^{2, *}$, G. Morelli ${ }^{4, *}$ and G. Rosenman ${ }^{2, *}$ \\ 1 Faculty of Engineering, Holon Institute of Technology, Holon 581200, Israel; apter@hit.ac.il (B.A.); \\ handelmana@hit.ac.il (A.H.) \\ 2 School of Electrical Engineering, Tel-Aviv University, Tel Aviv 69978, Israel; \\ lapshina.nadezda@gmail.com (N.L.); hani.barhom@gmail.com (H.B.) \\ 3 Faculty of Science, Holon Institute of Technology and School of Chemistry, Holon 581200, Israel; \\ fainberg@hit.ac.il \\ 4 Department of Pharmacy, Research Centre on Bioactive Peptides (CIRPeB), University of Naples \\ “Federico II", 80138 Naples, Italy; antonella.accardo@unina.it (A.A.); carlo.diaferia@unina.it (C.D.) \\ * Correspondence: pginzburg@tauex.tau.ac.il (P.G.); giancarlo.morelli@unina.it (G.M.); \\ gilr@eng.tau.ac.il (G.R.)
}

Received: 23 June 2020; Accepted: 22 July 2020; Published: 3 August 2020

\begin{abstract}
Nanoscale optical labeling is an advanced bioimaging tool. It is mostly based on fluorescence (FL) phenomena and enables the visualization of single biocells, bacteria, viruses, and biological tissues, providing monitoring of functional biosystems in vitro and in vivo, and the imaging-guided transportation of drug molecules. There is a variety of FL biolabels such as organic molecular dyes, genetically encoded fluorescent proteins (green fluorescent protein and homologs), semiconductor quantum dots, carbon dots, plasmonic metal gold-based nanostructures and more. In this review, a new generation of FL biolabels based on the recently found biophotonic effects of visible FL are described. This intrinsic FL phenomenon is observed in any peptide/protein materials folded into $\beta$-sheet secondary structures, irrespective of their composition, complexity, and origin. The FL effect has been observed both in natural amyloid fibrils, associated with neurodegenerative diseases (Alzheimer's, Parkinson's, and more), and diverse synthetic peptide/protein structures subjected to thermally induced biological refolding helix-like $\rightarrow \beta$-sheet. This approach allowed us to develop a new generation of FL peptide/protein bionanodots radiating multicolor, tunable, visible FL, covering the entire visible spectrum in the range of 400-700 nm. Newly developed biocompatible nanoscale biomarkers are considered as a promising tool for emerging precise biomedicine and advanced medical nanotechnologies (high-resolution bioimaging, light diagnostics, therapy, optogenetics, and health monitoring).
\end{abstract}

Keywords: bioimaging; fluorescence labels; fluorescent dyes for imaging of amyloid fibrils; peptide-based materials; PEGylated peptides; peptide nanostructures; peptide aggregates; thermally induced refolding helix-like $\rightarrow \beta$-sheet and intrinsic visible fluorescence in amyloid and amyloidogenic $\beta$-sheet nanostructures; fluorescence in polymer/peptide thin films and fibers; reabsorption mechanism of fluorescence long-range propagation; visible fluorescent bionanodots

\section{Introduction}

Molecular fluorescence (FL) is a process wherein an electronic relaxation is associated with a strong photon emission. Since its first observation, dating back to the 16th century, this optical process was the subject of intensive investigations, which continue to attract attention nowadays. 
From a quantum mechanical standpoint, FL is a radiative recombination process wherein an excited electron undergoes a dipolar-allowed transition to a ground state. This research has gained additional momentum, inspired by a wide range of emerging applications in the field of biology and biomedicine. Quantitative fluorescent microscopy made it possible to get a deep understanding and analyze a variety of basic fine biological processes, including direct imaging of living biocells and transportation of drug molecules in human tissues using FL biomarkers. A broad range of FL organic molecular dyes covering the entire visible spectrum has been discovered along with the recent development of another group of FL nanoscale labels such as semiconductor inorganic quantum dots, carbon dots, lanthanide ions and more. Intensive use of fluorophores of different origins in biomedical studies shifts accents to additional requirements, putting biocompatibility and biodegradability at a cornerstone. Those specifications made entire molecular families, e.g., rhodamine, and quantum dots to be inapplicable for a range of in vivo studies. The discovery of the first biocompatible fluorophore, extracted from a jellyfish, i.e., green fluorescent protein (GFP), was awarded a Noble Prize in 2008 and started a new era in bioimaging. Quite a few GFP derivatives were explored and allowed scientists to perform high-quality imaging with these biocompatible labels.

Recently, the family of label-free natural fluorescent bionanomaterials has obtained an additional representative, emerged after an unexpected observation of strong broadband FL emission both in natural amyloid fibrils related to neurodegenerative diseases (Alzheimer's, Parkinson's and more) [1], and bioinspired synthetic ultrashort di- and tri-peptides fiber structures [2]. Despite a deep difference in their composition and origin, both amyloid and amyloidogenic fibrillary structures are self-assembled into the same $\beta$-sheet architecture and exhibit similar excitation and FL photon emission spectra, making this new FL effect fold-sensitive.

In a living human brain, metastable soluble $\alpha$-helical amyloid proteins are gradually folded into thermodynamically stable, solid-state fibrillary $\beta$-sheet structures. We found that native amyloidogenic synthetic peptide/protein can be also transformed into $\beta$-sheet structure when subjected to thermally induced refolding helix-like $\rightarrow \beta$-sheet. This new method allowed us to develop universal nanotechnology for the transformation of any non-FL peptide/protein to $\beta$-sheet FL structure.

In this review, traditional FL biolabels and methods of FL bioimaging of amyloid fibrils are described. The main focus is directed toward basic studies of highly specific and new types of FL phenomena found in amyloid and amyloidogenic peptide/protein nanostructures and newly developed hybrid polymer/peptide thin films and fibers. We demonstrate a new mechanism of long-range FL propagation in poly(ethylene glycol) (PEG)-F6 amyloidogenic fibers.

We developed a new approach to visible FL nanodot fabrication. These nanodots are self-assembled from synthetic peptide/protein molecules. They are considered elementary building blocks of supramolecular bionanostructures. Originally, these peptide/protein synthetic molecules of biological origin and their bioinspired structures did not exhibit visible FL. We revealed that visible FL, covering the entire visible region $400-700 \mathrm{~nm}$, can be acquired by any peptide/protein nanodots as a result of the reconstruction of their native peptide/protein conformation from the primary $\alpha$-helical state to $\beta$-sheets by the use of thermally induced refolding.

We show that these novel FL visible nanodots of biological origin can be widely used in nanobiotechnology for medical imaging, diagnostics and therapy, development of a new generation of drugs and safety technology for cosmetics and food products, as well as dramatically impacting additional nanotechnological fields (image sensors, solar cells, light-emitting devices such as biolasers, and more). These studies pave the way for the development of a new research field of peptide photonics and advanced nanotechnology of peptide-integrated optics.

\section{Materials and Methods}

Bioinspired nanostructures are self-assembled from chemically synthesized biomolecules into diverse morphologies such as nanodots, nanotubes, nanotapes, thin films, fibers, and more. In this chapter, we consider various methods for their fabrication, deposition technology, and high-resolution 
experimental tools which allowed us to study their morphology at the nanoscale, their biochemical composition, biomolecular arrangement (peptide/protein secondary structure), their basic biological transformation from helix-like to $\beta$-sheet state, as well as their photophysical properties (optical absorbance, fluorescence, and more).

\subsection{Peptide Materials and Fabrication of Peptide Structures}

A few different peptide biomolecule monomers (Bachem, Switzerland) were applied for the fabrication of diverse peptide nanostructures: linear aromatic diphenylalanine (FF), linear aliphatic dileucine (LL), tri-phenylalanine (FFF) and insulin protein. In the native phase, these peptide molecules were self-assembled into various morphological shapes by the use of colloidal technology: linear FF and linear LL were self-assembled into hollow peptide nanotubes (PNT); linear FFF was self-assembled into nanospheres (PNS) or to plain wafers (tapes), depending on the solvent [2] Linear FF, FFF, LL, and insulin protein were also self-assembled into nanodots [3]. Peptide fiber structures were fabricated by two methods: under the thermal heating of peptide nanotubes in an oven and in a vacuum chamber by vapor deposition technology (the details can be found below).

\subsection{Preparation of Peptide Nanostructures}

L-diphenylalanine (FF) peptide nanotubes were self-assembled by dissolving the FF peptide powder in 1,1,1,3,3,3-hexafluoro-2-propanol (HFIP) (Sigma Aldrich) to initial concentrations of $100 \mathrm{mg} / \mathrm{mL}$, which were then mixed in a vortex mixer (VELP Scientifica) and further diluted to final concentrations of $2 \mathrm{mg} / \mathrm{mL}$ in deionized water. After vortexing, the solution drops from the peptide solution were deposited on a glass substrate and left to dry under a chemical hood. This fabrication process yields long, horizontally aligned FF peptide nanotubes with a hexagonal cross-section.

L-dileucine (LL) peptide nanotubes were self-assembled by first dissolving the LL peptide powder in HFIP to initial concentrations of $100 \mathrm{mg} / \mathrm{mL}$, which were then mixed in a vortex mixer and further diluted to a final concentration of $2 \mathrm{mg} / \mathrm{mL}$ in deionized water. After vortexing, the solution drops from the peptide solution were deposited on a glass substrate and left to dry in a chemical hood. This fabrication process yields long, horizontally aligned LL peptide nanotubes with an orthorhombic cross-section.

L-triphenylalanine (FFF) peptide micro-nanospheres were prepared by dissolving the FFF peptide powder in HFIP to an initial concentration of $100 \mathrm{mg} / \mathrm{mL}$, then mixed in a vortex mixer and then further diluted to a final concentration of $4 \mathrm{mg} / \mathrm{mL}$ in chloroform. After vortexing, the solution drops from the peptide solution were deposited on a glass substrate and left to dry in a chemical hood. This fabrication process yields micro-nanometer FFF spheres.

FFF peptide tapes with a large area were prepared by dissolving the FFF peptide powder in HFIP to an initial concentration of $100 \mathrm{mg} / \mathrm{mL}$, which was then mixed in a vortex mixer and further diluted to final concentrations of $2 \mathrm{mg} / \mathrm{mL}$ in deionized water. The FFF peptide biomolecules were self-assembled in aqueous solution and deposited on glass substrates with the use of a "pull-out" method combined with a strong modification of the surface substrate wettability. These methods enabled the fabrication of the FFF wafers with lengths of 5-100 $\mu \mathrm{m}$, widths of 5-30 $\mu \mathrm{m}$, and thicknesses of $0.2-1.5 \mu \mathrm{m}$.

FF and FFF peptide nanodots (native phase) were prepared by the following procedure: the lyophilized powder of FFF and FF (Bachem, Switzerland) was dissolved in HFIP to an initial concentration of $100 \mathrm{mg} / \mathrm{mL}$, mixed in a vortex mixer (VELP Scientifica) and then further diluted to a final concentration of $5 \mathrm{mg} / \mathrm{mL}$ either in acetone (Merck Millipore) or in $1 \mathrm{mg} / \mathrm{mL}$ in ethylene glycol (EG) (Sigma-Aldrich). Both organic solvents are related to a polar group which inhibits the self-assembly process at the stage of the nanodots. The dots are well dispersed and do not assemble into any large supramolecular structures such as tubes, fibers, plates, and more.

Visible fluorescence FF and FFF peptide nanodots were prepared by heating the native solutions in the ethylene glycol from room temperature up to $180^{\circ} \mathrm{C}$ (at this final temperature, the heating was 
fixed for three hs) and then cooled down back to room temperature. EG was chosen as a solvent because of its high boiling temperature point of $195^{\circ} \mathrm{C}$ [3].

\subsection{Preparation of Peptide Fibrillary Nanostructures}

The fabrication of thermally induced fibrillary nanostructures was performed by two methods:

1. The native FF, LL tubular, FFF spherical and FFF tape samples were heat treated in the oven using a step by step procedure from room temperature to $180^{\circ} \mathrm{C}$ (at every temperature step the peptides structures were heated for $1 \mathrm{~h}$ ) and then cooled back to room temperature to study their properties. The thermal treatment led to a deep modification of the native morphology, giving similar elongated close- and open-end fibrillary structures of $10-100 \mathrm{~nm}$ in diameter and $100 \mathrm{~nm}-30 \mu \mathrm{m}$ in length [2].

2. The method of vacuum vapor deposition of peptide biomolecule monomers resulted in the deposition of vertically aligned nanofibers. In a typical procedure, $1 \mathrm{mg}$ of FF peptide lyophilized powder was placed in a small copper boat to serve as the source material. The substrate (carbon, glass, gold, and silicon dioxide) was placed above the source at a vertical distance of $2 \mathrm{~cm}$. The chamber was then set to $220^{\circ} \mathrm{C}$ with a heating rate of $10^{\circ} \mathrm{C} \mathrm{min}^{-1}$ at a constant pressure of $10^{-5} \mathrm{mbar}$ [4].

\subsection{Experimental Methods}

\subsubsection{Secondary Electron Microscopy (SEM)}

All the above samples were inspected on silicon substrates, coated with gold-palladium, and scanned by TESCAN VEGA3 (Brno-Kohoutovice, Czech Republic), scanning electron microscope in a high vacuum.

\subsubsection{Spectrophotometry (Optical Absorbance)}

The optical absorption (OA) measurements were performed with a Cary 5000 UV-vis-NIR spectrophotometer (Varian, a part of Agilent Technologies, USA), over the range of 200-800 nm. For the OA measurements, all peptide-based nanostructures were prepared by the following procedure. The peptide samples containing either native or thermally induced peptide nanostructures were gently scrubbed into clean quartz cuvettes, which were then filled with deionized water. OA measurements were performed with these water suspensions.

\subsubsection{Optical Spectrofluorometry}

Measurements of fluorescence (FL) and fluorescence excitation (FLE) were performed using a Horiba Jobin Yvon FL3-11 spectrofluorometer (HORIBA Jobin Yvon S.A.S., 91165 Longjumeau cedex, France). The peptide nanostructures were placed on a $1 \times 1 \mathrm{~cm}$ quartz surface, and the FL measurements were conducted with a homemade holder.

\subsubsection{Fluorescence Microscopy}

Fluorescence images of FF, LL, and FFF nanostructures were obtained with an Olympus BX51WI (Microscopy Supplies and Consultants Ltd., Carnock, Fife, Scotland, KY12 9JH) fixed-stage upright fluorescence microscope. Samples were placed on clean coverslip glass, dried, and imaged with X10 Olympus objective. For blue images, a DAPI filter was used, and for green images-a GFP filter.

\subsubsection{Circular Dichroism (CD) Spectroscopy}

Circular dichroism (CD) spectra were obtained by a Chirascan CD Spectrometer (Applied Photophysics, United Kingdom). The wavelengths, from 190 to $250 \mathrm{~nm}$, were scanned every four seconds. The fabrication procedure for both native and thermally induced phases was identical to the fabrication process for the OA measurements. The final water suspensions of all peptide nanostructures were studied. 


\subsubsection{Time-of-Flight Secondary Ion Mass Spectroscopy (ToF-SIMS)}

The fabrication process of the samples for ToF-Secondary Ion (SI)MS measurements was identical to that mentioned in the SEM section. All the samples in the native and thermally induced phases were placed on a clean silicon substrate and measured with a PHI Model 2100 TRIFT II instrument, (CAE, Austin, TX 78701, USA).

\subsubsection{FTIR Measurements}

FTIR data were collected with the use of a Bruker Tensor 27 (MA, USA). First, a $3 \times 3 \mathrm{~cm}$ silicon-substrate reference sample was tested. Then, droplets of the peptide suspensions were placed on the silicon substrates and dried at room temperature. Then, FTIR measurements of the native peptide nanostructures and heat-treated peptide fibrillary samples were performed.

\section{Fluorescence Phenomena and Imaging Biomarkers}

Bioimaging is a principal method in biomedicine from local diagnosis and drug delivery to therapy and surgery [5,6]. It is commonly based on fluorescent (FL) agents of high brightness and combines their exceptional optical properties with biocompatibility, biodegradability, and precise targeting both in vivo and in vitro. The FL mechanism of any fluorophore represents a quantum process, including the stimulated absorption of incident light photons of one wavelength, which is followed by the emission of FL photons with shifted wavelength. Diverse FL light-responsive materials of different origins such as fluorescent proteins [7,8] organic molecular dyes [9], inorganic semiconductor quantum dots [10], and carbon nanodots [11] were developed (Figure 1, Table 1). Each of them has its own inherent mechanism of optical absorption and FL photon emission, which defines its key figures of merit for bioimaging: FL spectral region (wavelength of FL maximum and its full width at half maximum (FWHM), FL quantum yield, Stokes shift, and biocompatibility.

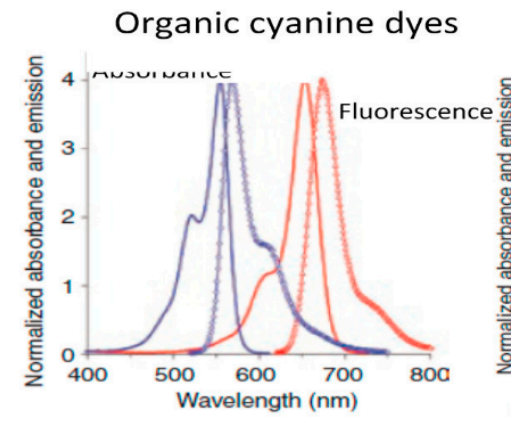

(a)

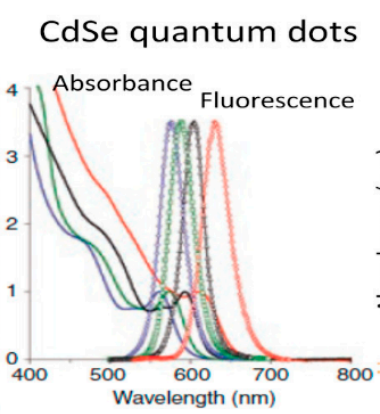

(b)

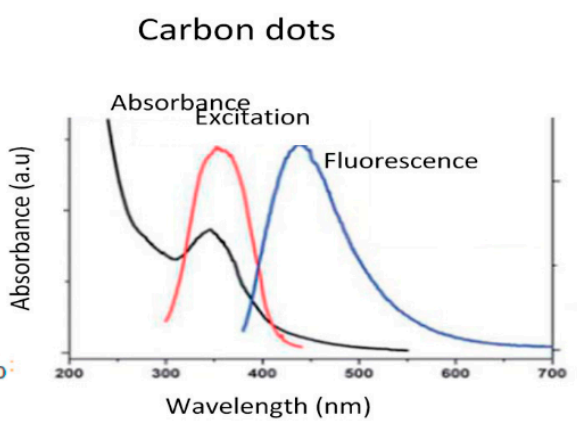

(C)

Figure 1. Optical absorption and FL spectra of organic dyes, quantum dots, and carbon dots. (a) Absorption (lines) and FL emission (symbols) spectra of representatives of organic dyes Cy3 (blue) and Cy5 (red) characterized by a resonant spectrum of both absorbance and FL; (b) Absorption (lines) and FL (symbols) of CdSe quantum dots, absorbance spectra, and FL red shift is observed for QDs with the larger size in the range of (3.5-6.0) nm; (c) schematic UV-visible and FL spectra of carbon dots. (Taken from [12,13]).

Fluorescent proteins: Currently, FL proteins are shown to be the most frequently used FL probes [14]. Green FL protein (GFP), the first and most famous FL protein, was discovered and extracted from jellyfish, Aequorea victoria, in the 1960s [15]. GFP are the only molecules, to date, that are known to have visible FL of biological origin among an unlimited number of non-FL peptide and protein biomolecules [7,8]. Since the 1990s, GFP has been genetically engineered to produce a vast number of mutants. Unlike other molecular-based probes, FL proteins can be genetically coded and label cellular structures [8]. GFP and homologs with different compositions cover the entire visible and 
infrared spectrum. They are classified [8] as ultraviolet, blue, cyan, green, yellow, orange, red, far red, and near infrared proteins. These proteins exhibit discrete optical absorbance bands, relatively narrow FL spectra of (50-100) $\mathrm{nm}$ bandwidth, and large Stokes shifts of $\sim 100 \mathrm{~nm}$. Their quantum yield reaches $98 \%$ (Table 1), which makes these originally biocompatible FL tags the most effective bioprobes.

Organic molecular dyes: FL compounds based on synthetic large molecules are powerful tools to visualize biological processes in living cells and organisms. Ever since the discovery of organic FL dyes in the late 19th century, efforts have been made to study the behavior of specific biomolecules in living systems by using these dyes as biolabels [16]. Many organic bright and photostable compounds such as fluorescein, rhodamine, coumarin, and many other dyes were developed. The FL spectra of these diverse visible molecular dyes supply labels for almost the entire visible and near-IR spectrum (Table 1, Figure 1a) [17]. Some of them were later employed for optical bioimaging as local FL labels and probes owing to their FL high quantum yield. The majority of common fluorophores such as fluorescein, rhodamine, and cyanine are resonant FL dyes that are characterized by narrow optical absorption of (25-35) nm and FL emission bands with small Stokes shifts of $~ 25-30 \mathrm{~nm}$ (Figure 1a), high molar absorption coefficients, and moderate-to-high fluorescence quantum yields (60-97)\% [17]. Another group of organic dyes (Table 1, Figure 1a) displaying high FL intensity and long FL lifetimes includes cyanine, squaraine, phthalocyanines, porphyrin, and boron-dipyrromethene (BODIPY) dyes with red to near-infrared (NIR) FL $[18,19]$. They are especially in demand for in vivo imaging in animals and humans, owing to the transparency of tissues in this NIR wavelength region, providing cancer targeting and imaging. Recently, a breakthrough was achieved with produced chemically modified organic small-molecule cyanine dyes [20]. The wavelengths of light-absorbed and FL-emitted photons by these new dyes are shifted roughly $200 \mathrm{~nm}$ towards longer wavelengths, that is, towards an infrared region greater than $1000 \mathrm{~nm}$. Imaging in this spectral range offers important benefits for in vivo applications compared with that in the far red regions [21].

Quantum dots (QDs): Semiconductor QDs are the nanoparticles that bridge the gap between individual atoms and bulk semiconductor materials [10]. The electronic structure of semiconductors at the nanoscale is size dependent (Figures $1 \mathrm{~b}$ and 2). This effect is known as quantum confinement (QC) and it is determined by the tough limitation of the free motion of electrons in a restricted number of dimensions. Schematic representation of the quantum confinement $(\mathrm{QC})$ effect is shown in the Figure 2.

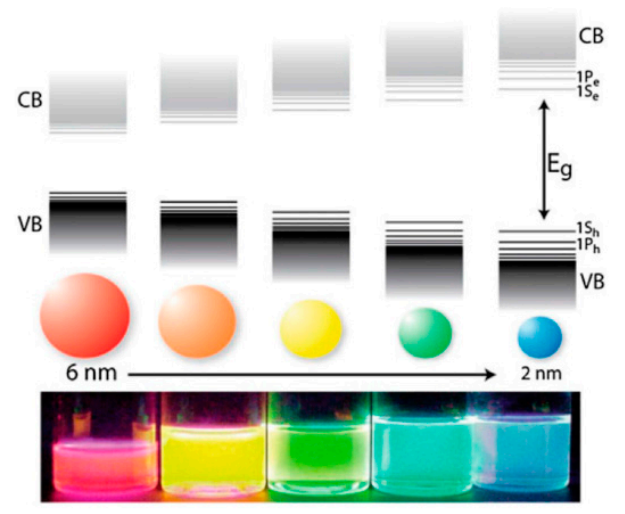

Figure 2. Schematic representation of the quantum confinement (QC) effect: the bandgap of a semiconductor material increases with decreasing size, and discrete energy levels arise at the band edges. Lower panel shows a photograph of five FL colors CdSe quantum dots (QDs) with different sizes under excitation with a UV lamp, exhibiting the tunability of FL spectra. (Taken from [22]).

The dimensions of the confined structural regions are defined by a critical quantum size, the exciton Bohr radius. Conceptually, it represents the average distance between the photoexcited electron-hole pair, which is called an exciton. Under the condition in which the physical size of QDs approaches that of the exciton (for example, for CdSe exciton, the radius is about $6 \mathrm{~nm}$ ) the energy gap of the 
semiconductor material increases with decreasing size and discrete energy levels arise at the band edges. This means that the optical energy gap of nanoparticles and, consequently, the FL spectra of the QDs can be altered by adjusting their size [23], leading to a wide range of FL tunability (Table 1, Figures $1 \mathrm{~b}$ and 2 ).

Table 1. Comparison of photonic properties of FL proteins, organic dyes, inorganic quantum dots, and carbon dots [12,16-23].

\begin{tabular}{|c|c|c|c|c|}
\hline $\begin{array}{l}\text { Photonic } \\
\text { Properties }\end{array}$ & $\begin{array}{l}\text { GFP and } \\
\text { Homologs, } \\
\text { Natural FL } \\
\text { Proteins }\end{array}$ & Organic Dyes & $\begin{array}{c}\text { Quantum Dots } \\
\text { Size-Dependent } \\
\text { Data }\end{array}$ & Carbon Dots \\
\hline $\begin{array}{l}\text { Optical absorption, } \\
\mathrm{nm}\end{array}$ & $\begin{array}{l}\text { Peak(365-600) nm } \\
\text { depends of } \\
\text { composition }\end{array}$ & $\begin{array}{c}\text { Discrete optical } \\
\text { absorbance bands: } \\
\text {-Fluorescein, max } \\
\sim 500 \mathrm{~nm} \text {, } \\
\text { FWHM 35 nm } \\
\text {-Cyanine, Cy3 } \\
\text { max } 550 \mathrm{~nm}, \\
\text { FWHM 35 nm }\end{array}$ & $\begin{array}{c}\text { Broadband } \\
\text { absorbance } \\
\text { (size-dependent), } \\
\text { steady increase } \\
\text { toward UVfrom } \\
\text { absorption onset, } \\
\text {-CdS 350-470 nm } \\
\text {-CdSe 450-640 nm } \\
\text {-InP 550-650 nm }\end{array}$ & $\begin{array}{l}\text { Broadband } \\
\text { absorbance, in the } \\
\text { UV region with a } \\
\text { tail extending to } \\
\text { the visible range }\end{array}$ \\
\hline $\begin{array}{l}\text { Fluorescence } \\
\text { spectra, nm }\end{array}$ & $\begin{array}{l}\text { Peak(424-620) nm } \\
\text { depends of } \\
\text { composition }\end{array}$ & $\begin{array}{l}\text { Resonant narrow } \\
\text { spectra: } \\
\text {-Fluorescein, } \\
\text { max } \sim 550 \mathrm{~nm}, \\
\text {-Cyanine, Cy3 } \\
\text { FWHM 34 nm }\end{array}$ & $\begin{array}{l}\text { FL optical region } \\
\text { (size-dependent), } \\
\text { narrow spectra: } \\
\text {-CdS 370-500 nm } \\
\text {-CdSe 470-660 nm } \\
\text {-InP } 620-720 \mathrm{~nm}\end{array}$ & $\begin{array}{c}\text { Excitation } \\
\text { dependent FL, FL } \\
\text { set of separated } \\
\text { peaks in the visible } \\
\text { region }\end{array}$ \\
\hline $\begin{array}{c}\text { Fluorescence } \\
\text { spectra, FWHM, } \\
\text { nm }\end{array}$ & $(50-100) \mathrm{nm}$ & $\begin{array}{l}\text {-Fluorescein, } \\
\text { FWHM 34 nm } \\
\text {-Cyanine, Cy3 } \\
\text { FWHM 34 nm }\end{array}$ & $\begin{array}{c}-\mathrm{CdS} \sim 30 \mathrm{~nm} \\
-\mathrm{CdSe} \sim 30 \mathrm{~nm} \\
-\mathrm{InP} \sim 50-90 \mathrm{~nm}\end{array}$ & 100-150 nm \\
\hline Quantum yield,\% & $\begin{array}{l}\text { QY is varied in a } \\
\text { wide range and } \\
\text { depends on the } \\
\text { composition } \\
(20-98) \%\end{array}$ & $\begin{array}{c}\text {-Fluorescein, } \\
\text { can reach } 97 \% \\
\text {-Cyanine, Cy3 } \\
40 \%\end{array}$ & $\begin{array}{c}-\mathrm{CdS}<60 \% \\
-\mathrm{CdSe} 65-0.85 \% \\
-\operatorname{InP} 10-60 \%\end{array}$ & $\begin{array}{c}\text { Strongly depends } \\
\text { on technology of } \\
\text { preparation and } \\
\text { doping: varies in a } \\
\text { very large range } \\
(4-90) \%\end{array}$ \\
\hline Stokes shift, nm & $\begin{array}{l}\text { Widely varied, can } \\
\text { reach } \sim 100 \mathrm{~nm}\end{array}$ & Normally $<50 \mathrm{~nm}$ & $\begin{array}{c}<50 \text { nm for visible } \\
\text { wavelength }\end{array}$ & - \\
\hline
\end{tabular}

The FL of QDs demonstrates a narrow-band resonant-like FL of 30-50 nm width (Figure 1b, Table 1). In comparison to organic dyes, QDs have completely different properties of a step-like continuous broadband optical absorption starting at the absorption onset and defined by the size of the dots. It gradually increases toward the shorter wavelengths (Figures $1 \mathrm{~b}$ and 2). The broad absorption allows for the free selection of the excitation wavelength and thus the straightforward separation of excitation and emission [12]. QDs are photostable and have an excellent molar extinction coefficient that is more than 10 times larger than that of organic dyes and demonstrates a very bright FL light. A severe limitation of QDs in biomedical applications is related to their cytotoxic effects because of their chemical compositions, which are based on cadmium, lead, mercury, arsenic elements. However, QDs can be labeled with various ligands, peptides, and antibodies, and thus they were successfully used in biological imaging, cancer diagnosis, drug monitoring, and delivery applications [24,25].

Carbon nanodots: Carbon dots are a relatively new class of carbon nanomaterials that have been intensively studied in the last fifteen years. Carbon sources used for their fabrication range from graphite, proteins, and food products. Citric acid is the most commonly used precursor. Carbon 
dots, in contrast to semiconductor QDs, lack a classical bandgap structure. They can be constructed to achieve bandgap-like fluorescence via surface passivation-created defects. CDs typically show optical absorption in the UV region, with a tail extending to the visible range (Figure 1c). Their optical properties $[13,26]$ drew particular interest as they display the unusual trait of excitation-dependent emission, providing FL spectra with a broad color range and emission peak wavelengths ranging from 415 (violet) to $615 \mathrm{~nm}$ (orange-red) and higher, as well as high FL quantum yields reaching 80-90\%, long photoluminescence decay lifetimes, and photostability in doped carbon dots (Table 1 , Figure 1c) $[13,26-28]$. The FL mechanism of carbon dots is still under discussion, mainly regarding the surface state-controlled defects. These defects can modulate the color of FL as well as create a heterogeneous electronic environment resulting in pronounced excitation wavelength-dependent emission. Since they were discovered, carbon dots have been viewed as a less toxic alternative to traditional quantum dots and as very promising for applications in bioimaging and biosensing $[27,28]$.

A few major types of FL bioimaging labels can be roughly divided into two groups: molecular labels (bioorganic natural GFP and homologs, synthetic organic molecular dyes) and nanodot labels (carbon and inorganic quantum dots). The molecule-based FL agents demonstrate similar physical origins of FL generation, based on classic selective electron transitions. The electronic structure of these molecular tags provides composition-sensitive optical properties. They are characterized by a highly specific peak-like optical absorbance band and narrow FL spectrum for each type of biological or synthetic molecule.

The physics of FL in crystalline inorganic quantum nanodots obeys the quantum size effect, where the FL spectrum is size-dependent. The FL optical band can be widely varied by changing the semiconductor dot dimension (Table 1, Figure 2). Carbon dots irradiate FL photon emission, owing to surface state, defects, or doping. The FL spectrum, defined by surface and defect states, strongly depends on composition and preparation technology and can be varied in a very wide range.

In this paper, we present the basic physics and technology of completely different types of FL phenomena and new-generation FL visible bionanodots found in amyloid and amyloidogenic peptide/protein biological and bioinspired nanostructures. This new biophotonic effect (FL excitation and emission spectra) is similar for all peptides/proteins self-assembled into $\beta$-sheet secondary structures.

\section{Fluorescent Dye Imaging of Amyloid Fibrils}

Amyloid plaque formation is a key event related to several neurodegenerative misfolding disorders. Amyloid assembly is a multistep process initiated by conformational changes in monomeric species. The cascade hypothesis involves different intermediate architectures, including oligomers, protofibrils, filaments, and fibrils, generated upon the progressive self-assembling [29]. The amyloid mechanism aggregation comprises three steps: the lag phase, in which the monomers, low molecular weight oligomers, are the preeminent species, the polymerization, where the monomers mostly self-assemble generating aggregates with higher molecular weight, and the final saturation, where fibers-finally formed-evolve into a mature, self-assembled system.

Biologically, it has been widely supposed that soluble intermediate aggregates are the most toxic species, rather than mature fibrils [30]. From this perspective, the ability to monitor the kinetics of self-association result in a crucial clue to understanding the fibrillogenesis mechanism. Indeed, this knowledge represents essential data for learning about possible aggregation pathways as well as for the design and proposal of potential inhibitors. In line with these considerations, researchers' efforts have been devoted to developing analytical methodologies that are able to gain insights into toxic species formation. These approaches give a qualitative, quantitative and mechanistic understanding about the molecular events involved in fibrillogenesis.

In recent years, various techniques including staining [31], turbidity measurements [32], X-ray diffraction [33], light scattering [34], gel electrophoresis [35], sedimentation velocities [36] or size exclusion chromatography [37] have been widely proposed to this end. However, some of 
these methodologies are strongly limited by experimental conditions, such as the metastable nature of the oligomers, their low concentration, and their heterogeneous nature. Besides, the self-assembly kinetics are extremely dependent on the environment, including medium type, monomer concentration, temperature, $\mathrm{pH}$, metal ion presence, or ionic strength [38]. Optical techniques related to the use of fluorescent dyes can overcome some of these drawbacks, working at much lower concentrations with more sensitivity, in less time, and allowing the spatial distribution of the emitting species. In this context, the study of amyloid phenomena is strictly related to fluorescence label applications. In the study of amyloid kinetics, two different approaches are applied: in the first one, the association/dissociation of aggregates is monitored via an extrinsic dye, whereas, in the second one, monomeric peptides are chemically derivatized with fluorescent dye before their aggregation. In the latter approach, the aggregation is intrinsically studied.

\subsection{Fl Extrinsic Dyes and Imaging Technology of Amyloid Fibers}

Extrinsic fluorescent probes have the ability to bind the amyloid aggregates in a non-covalent way, changing their spectral fluorescence signature as a consequence of the interaction with amyloid species. The typical kinetics of the aggregation assay response, with a sigmoidal profile of the fluorescence intensity vs. time, are governed by the formation of larger and more mature aggregates. FL emission intensity is conceptually considered proportional to the percentage of dye molecules bound to fibrils and the number of self-assembled systems, which allow for the easy evaluation of the process kinetics and the aggregate concentration.

Traditionally, the most important and most widely used fluorescent organic dyes are Thioflavin $\mathrm{T}$ (ThT), Congo Red (CR), and 1-Anilino-8-naphthalene sulfonic acid (ANS). ThT (Figure 3, ThT) was first used with a synthetic mimicking the A $\beta$ peptide by Levine in 1993 [39]. ThT is a fluorescent cationic benzothiazole dye that binds itself to the structure channels on the surface of aggregates in a stabilized fashion. This phenomenon greatly enhances and shifts the fluorescence emission from 445 to $482 \mathrm{~nm}$ as a consequence of the loss of rotational freedom in the dye structure. ThT has been efficiently applied to study the kinetic behavior of $A \beta$ polypeptides [40] or for the recognition of $\beta$-sheet organization in peptide-based materials [41] and hydrogels [42].

Nevertheless, some limitations affect ThT application. It is well known that the binding behavior of ThT dye is not only selective for amyloid aggregates. Indeed, ThT can also detect keratin, elastin fiber, and nucleic acids [43]. Furthermore, different conformational and oligomeric states are not properly detected and ThT self-aggregates in micelles starting from $4 \mu \mathrm{M}$ concentrations [44]. Due to the positively charged quaternary heterocyclic nitrogen of the benzothiazolium, ThT is not suitable to cross the blood-brain barrier (BBB) and the charged nature of the molecule limits its use in neutral, basic conditions [45].

Due to these drawbacks, several alternative dyes have been studied as potential fluorescent amyloid probes. For example, NIAD-4 [5'-(4-hydroxyphenyl)-[2,2'-bithiophen]-5-yl] methylene]-propane dinitrile (Figure 3, NIAD-4), designed by Swager and co-workers, was efficiently employed for the recognition of $A \beta(1-40)$ aggregates [46]. This fluorophore was also used for monitoring in vitro the amyloid formation processes of de novo coiled-coil peptides [47]. The studied peptides can adopt different conformations in acid and basic environments. Specifically, Peptide 1 forms amyloid-like fibrils at $\mathrm{pH} 4$, whereas thin micrometer long $\alpha$-helical fibers were found in physiological conditions $(\mathrm{pH}=7.4)$. On the other hand, Peptide 2 shows the reverse supramolecular behavior, forming amyloid aggregates at $\mathrm{pH} 7.4$ and $\alpha$-helical fibers in acidic conditions. The fluorescence signal of the dye $\left(\lambda_{\mathrm{ex}}=475 \mathrm{~nm} ; \lambda_{\mathrm{em}}=600 \mathrm{~nm}\right)$ was detected only for the amyloid forms of the studied peptides, whereas no significant emission was registered for the helical fibers. This evidence suggests an increase in the specificity of NIAD-4 concerning ThT in acidic conditions. Beyond NIAD-4 dye, three other probes (L1, L2, and L3 reported in Figure A3) with a molecular structure similar to ThT were proposed by Dyrager and co-workers [48] for the detection and visualization of $A \beta 1-42$ plaques in transgenic mice 
brain slices. Nevertheless, despite the capability of these dyes to emit significant photoluminescence quantum yields, they did not exhibit the expected "turn-on" features.

An alternative method for the detection of amyloid structures is the Congo Red (CR) assay. CR (Figure 3, Congo Red), the sodium salt of 3,3'-([1,1'-biphenyl]-4,4'-diyl)bis(4aminonaphthalene-1-sulfonic acid) is a heterocyclic and aromatic water-soluble diazo-dye used especially as a $\mathrm{pH}$ indicator and as a biological staining agent of amyloids. The $\mathrm{CR}$ staining procedure is a qualitative method applied for the macroscopic identification of amyloid deposits both in vitro and in tissue. As reported for ThT, CR became torsionally restricted when bound to amyloid deposits, adopting a specific orientation with the long molecular axis of the dye running parallel to the fibril one. The in vitro binding of $C R$ to amyloids induces a characteristic increase in the $C R$ absorption spectrum associated with a bathochromic effect from 480 to $\sim 512 \mathrm{~nm}$ and the appearance of an additional shoulder centered at $540 \mathrm{~nm}$ [49]. The specific orientation of CR dye in the presence of natural or synthetic $\beta$-sheet rich materials also provides a birefringent anisotropic light transmission effect ("apple-green birefringence") under polarized light [50,51]. Analogously to the ThT derivative, many other CR-based aniline dyes were designed and proposed as fluorescent dyes for amyloid testing. This class includes Congo Corinth (Figure 3, Congo Chorint), Vital Red, and Acid Fuchsin. The detection in solution of several amyloid aggregates (including $\alpha$-synuclein and tau) was achieved using the CR derivative K114 (Figure 3, K114 (trans, trans)-1-bromo-2,5-bis(4-hydroxy)styrylbenzene) [52]. The fluorescence response for the in vivo detection was improved designing $C R$ analogues, including 1,4-bis(49-hydroxystyryl)-2-methoxybenzene (X-04) [53] and (trans,trans)-1bromo-2,5bis(3-hydroxycarbonyl-4-hydroxy)styrylbenzene (BSB) [54].

Partially folded intermediate aggregative states, having solvent-exposed hydrophobic clusters, can be detected using aggregation-induced emission probes, such as ANS (Figure 3, ANS) dye [55]. ANS has low fluorescence quantum yield in aqueous environments, whereas its emission is dramatically increased in non-polar ones, like self-assembling peptide spines [56]. An increase in emission is referred to as a consequence of dye binding to hydrophobic tasks. According to these considerations, ANS was evaluated as a useful fluorophore for kinetic studies, the determination of critical aggregation concentration and the equilibrium study of amyloids [57-59] It was also used to assess the influence of various external factors (i.e., concentration, $\mathrm{pH}$, presence of molecular inhibitors) on the assembly. ANS and its analog bis-ANS (Figure 3, ANS) were found to be capable of efficiently detecting the kinetics of $A \beta$ amyloid aggregation, while not affecting the morphologies, as demonstrated by TEM. However, both these fluorophores are not able to report the formation of oligomers [60]. Beyond ANS, the sodium salt of 1,2-bis [4-(3-sulfonatopropoxyl)phenyl]-1,2-diphenylethene (Figure 3, BSPOTPE), a tetraphenylethlyene (TPE)-based dye, was found able to detect insulin fibrillization in a real-time assay [61]. 


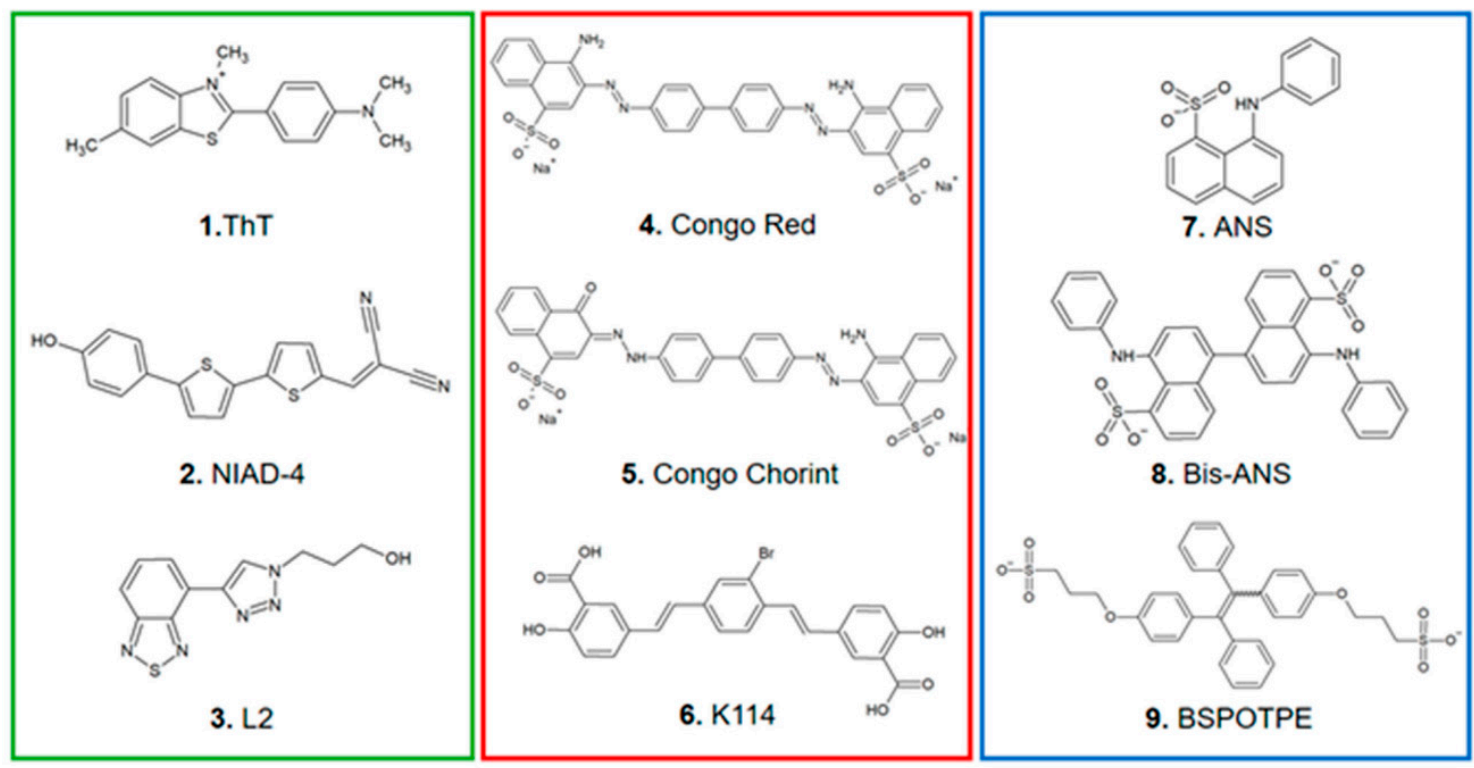

Figure 3. Chemical structure of some extrinsic fluorescent dyes used to follow amyloid fibril formation. (Taken from [59]).

\subsection{Direct Monomer FL Amyloid Labeling}

The efficiency of the extrinsic fluorescent methodology for amyloid study is strictly related to the lack of interaction/modification of the dyes. Nevertheless, an increasing number of papers on the behavior of dye in monomer aggregation events report on the detection of the effects of inhibition, which justify intensive investigations of these fluorophores for the observation of mature aggregates [62-65]. Moreover, most of the used dyes are not able to respond to oligomeric aggregates, showing FL signals only in the presence of mature fibrils. Thus, the development of probes for the detection of oligomers represents a topic of high importance. The direct labeling of aggregative monomers represents a feasible strategy in this research area. Indeed, the monomer tag has allowed the monitoring of amyloid formation at the nanoscale using microscopy technologies. In this case, chemical modification of the original peptide is required. Due to the low contribution of the $\mathrm{N}$-terminus during the peptide aggregation, this position is often identified as a suitable site for the labeling of the peptide. Monomers can be labeled using fluorescent organic dyes, complementary emitting units, modified amino acid residues, or FRET (fluorescence resonance energy transfer) probes. Several examples reported up to now are described below.

Using a stochastic optical reconstruction microscopy STORM (super-resolution microscopy technique) methodology, different types of amyloid architectures (oligomers and mature aggregates) were identified both in vitro and in vivo by Kaminski Schierle et al. [66]. In detail, the authors labeled $A \beta(1-40)$ with HiLyte Fluor 488 and studied the cellular uptake of the monomers. These results supported the hypothesis of a toxic role of intracellular $A \beta$ aggregates. The super-resolution microscopy technology, coupled with atomic force microscopy (AFM), was also applied to study the peptide surfactant self-aggregation process. In this study, Alexa Fluor 647 and Cy3B fluorophores were coupled on the side chain of the Lysine residue via NHS (N-Hydroxysuccinimide) ester conjugation chemistry [67]. In this case, a laser at $\lambda=647$ and $\lambda=561 \mathrm{~nm}$ activated the dyes, whereas the $405 \mathrm{~nm}$ surgent was applied in order to compensate the dye photobleaching by switching them between off and on states. The acquired two-color imaging of the samples helped in the interpretation of the mutual interactions of fibers. In addition, multicolor STORM imaging can be used to examine the monomer exchange dynamics.

The fluorescent tag also allowed the calculation of monomer-dimer equilibrium constants for $\mathrm{A} \beta 1-42(12.8 \mu \mathrm{M})$ and $\mathrm{A} \beta 1-40(54.0 \mu \mathrm{M})$ via modification of the N-terminus of the sequences 
with a tetramethylrhodamine (TMR)-modified lysine residue [68]. The self-quenching of the fluorophores allowed the monitoring of three distinct steps in the aggregation phenomenon, called the oligomerization, lag, and growth phase, respectively.

An alternative methodology to detect the oligomerization of the $\mathrm{A} \beta$ polypeptide was proposed by Hashimoto et al. [69] In this methodology, the $C$ - and N-terminus of the Gaussia luciferase were separately bounded to the $A \beta$ sequence. In the monomeric form, both these systems showed no luminescence features, but when both tags start to aggregate, the peptide-driven aggregation induces luminescence emission. Using this approach, stable oligomer populations (including trimer, octamers, and larger oligomers) were detected efficiently. The robustness of this method was previously demonstrated by Danzer et al. [70] and by Outeiro et al. [71], which were able to achieve direct cell imaging for $\alpha$-synuclein oligomers.

The early state of $A \beta(1-40)$ fibrillation was also explored using time-resolved and single-molecule FL experiments by Ott's research group [72]. In their studies, the authors conjugated several fluorophores like Atto 488, Atto 655, Hi-Lyte 488 and HiLyte 647 on the polypeptide sequence. Different distributions of oligomers in terms of dimensions were detected as a function of the specific dye properties. More specifically, dyes possessing a net attractive interaction with the peptide generated oligomers with a high molecular weight, which do not participate further in the formation of mature fibrils. On the contrary, low molecular weight oligomers, consumed during the aggregation phenomenon, tend to be formed by modified peptides containing fluorophores with less hydrophobic interactions.

FRET-based methods were also efficiently applied to follow the formation of fibrillary structures. A recombinant $\alpha$-synuclein ( $\alpha$-synuclein-C4) with a C-terminal tetracysteine additional sequence was designed and produced by Roberti et al. [73]. The C4 molecular portion allowed for monomer decoration with fluorogenic biarsenical compounds, FlAsH and ReAsH, two fluorescein-based dyes. In living cells, FlAsH/ReAsH FRET experiments confirmed the aggregation and allowed the study of fibrillogenesis related to the presence of other proteins in living cells, the response to cellular stress and the effect of drugs.

$A \beta$ oligomers were also detected using a FRET-based probe consisting of an A $\beta$-based peptide decorated with cyan (CFP) and yellow fluorescent protein (YFP) at its opposite ends. According to this molecular design, FRET can occur intramolecularly between the donor (CFP) and the acceptor (YFP) when the probe interacts with the oligomers. Moreover, the FRET event was used to estimate the $K_{d}$ $(8.2 \mathrm{nM})$ for oligomers [74].

Different $A \beta$ self-assembled forms were identified via FRET using a probe consisting of three dyes (ThT, sulforhodamine B (SRB), and sulfo-Cy5 (sCy5)) and a double copy of the FFVLK sequence, acting as a recognition motive of the $A \beta$ polypeptide [75]. Interacting with different aggregate forms, the probe exhibits different FL responses at $680 \mathrm{~nm}$ as a consequence of FRET efficiencies between the three dyes. In the study, a decrease was observed in the intensity emission peak at $680 \mathrm{~nm}$ in the presence of high molecular weight oligomers, whereas larger aggregates, like protofibrils and mature fibrils, cause an increase in the intensity of this peak. These results suggest that, according to this strategy, it is possible to detect the formation of intermediates. Finally, the detection of amyloid aggregates was also evaluated by the incorporation of specific fluorescent active residues such as cyanophenylalanine (FCN). This dye was incorporated in the sequence of human islet amyloid peptide (IAPP) instead of tyrosine and phenylalanine by Marek and co-workers [76]. The aggregation process was followed on the evidence that FCN exhibits significant FL when exposed to water environments. This approach was also applied in the shorter peptide $A \beta(1-23)$, punctually mutated with FCN in the positions F4, Y10, F19, and F20.

\section{Intrinsic Fluorescence in Amyloid Fibrils}

A great variety of human dysfunctional mental diseases such as Alzheimer's disease, Parkinson's disease, type II diabetes, and more are associated with the self-assembly of amyloid proteins into 
solid-state fibrillary nanostructures [77]. The mechanism of this fundamental amyloid transformation is considered as a deep refolding from the native soluble $\alpha$-helical state into $\beta$-sheet-rich elongated crystalline nanowires, amyloid fibrils [78-80]. It was shown that the native $\alpha$-helical state of some amyloid protein is not thermodynamically stable, and the proteins can lower its overall free energy through this structural transformation. Therefore, this amyloid fibrillary morphology is the most stable one that protein molecules can adopt under age-related physiological conditions [81]. Despite the native composition, size, and disease-related function, all aggregated amyloid structures share, at the nanoscale, a common structural fibrillary morphology. These amyloid fibers consist of highly ordered $\beta$-strands that run normally along axes of these elongated ultrathin fibrils and share with all amyloid structures the common protein secondary architecture of cross- $\beta$ sheet arrangement.

Another remarkable common property recently discovered in amyloid structures is the visible FL phenomenon. It was revealed in synthetic peptide structures derived from elastin and crystalline proteins with $\beta$-sheet secondary arrangements [82-84]. All of them displayed visible FL effects, which were ascribed to electron delocalization caused by hydrogen bonds in the $\beta$-sheet structure, providing low energy electronic transitions. Later, this new and unusual visible FL was disclosed in two different large classes of amyloid [1,85-88] and amyloidogenic [2,3,89-93] peptide/protein structures as well as in a new generation of hybrid polymer/peptide thin films and fiber materials $[94,95]$. All of them were folded into a $\beta$-sheet secondary structure.

A visible FL effect (Figure 4) was found in two amyloid proteins, $A \beta_{1-42}$ and $A \beta_{33-42}$, which represent both forms of typical $\beta$-sheet fibrils, where the former is aromatic and the latter lacks any aromatic amino acids [87]. Both fibrillary structures display the same emission spectra with a FL maximum near 440-470 $\mathrm{nm}$ under UV excitation at 365-380 $\mathrm{nm}$ (Figure 4). This novel class of fold-sensitive FL optical effects appears, owing to the dramatic modification of the peptide/protein electronic structure under the natural refolding helix-like $\beta$-sheet state.

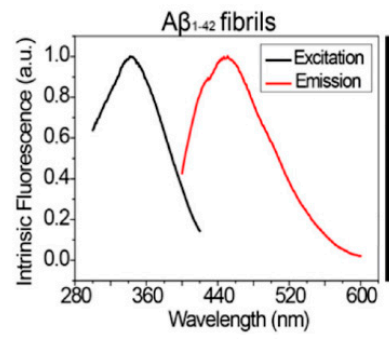

(a)

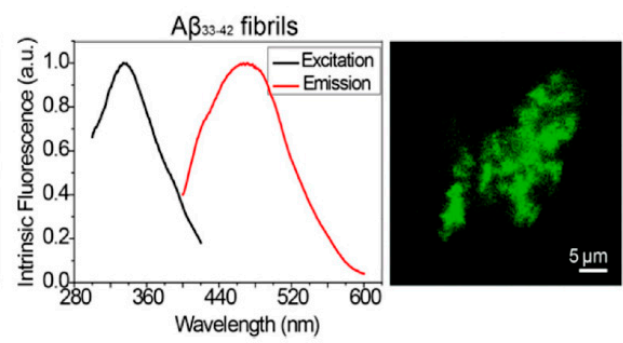

(b)

Figure 4. Protein fibrils display fluorescence properties. (a) Excitation and emission spectra of the intrinsic FL in aromatic $A \beta_{1-42}$ fibrils and corresponding FL image of unlabeled aggregates obtained using a confocal microscope. (b) Excitation and FL emission spectra of the intrinsic FL in non-aromatic $\mathrm{A} \beta_{33-42}$ fibrils and corresponding FL image. (Taken from [87]).

Experimental results obtained in a wide research from five different amyloid systems [1] A $\beta_{40}$ (a), $\mathrm{A} \beta_{42}(\mathrm{~b}), \mathrm{A} \beta_{33-42}(\mathrm{c}), \mathrm{K} 18$ tau (d) and I59T lysozyme (e) also showed that this universal FL is observed in all the $\beta$-sheet amyloid structures. Fluorescence microscopy images of these different amyloid fibrils emitting FL light in the deep blue wavelength range of $\sim 488 \mathrm{~nm}$ are presented in Figure 5.

This intrinsic FL is independent of the presence of aromatic side-chain residues within the polypeptide structure (Figure 5). Rather, it appears to result from electronic levels that become available when the polypeptide chain folds into a cross $\beta$-sheet scaffold. Moreover, these findings were used to quantify in vitro protein aggregation by FL imaging in a label-free manner. This novel property opens up an avenue for the direct exploration of amyloidogenesis in the human brain using a noninvasive and contrast agent-free imaging method. Indeed, this FL effect was used to track the aggregation of amyloid proteins noninvasively and quantitatively without any need for extrinsic labels [88]. Excitation-dependent multicolor FL in the range of $450-550 \mathrm{~nm}$ was found in amyloid 
fibers and spherulites fabricated from $\beta$-LG from bovine milk and bovine insulin [96]. Recently, the use of laser-induced fibril FL properties as a characterization method for biological protein secondary structures, in addition to convention circular dichroism, was proposed [97]. Moreover, this FL effect allows the direct observation of the seeding, nucleation, and growth of amyloid fibrils at the earliest stages of this amyloidosis (Figure 6) [85].

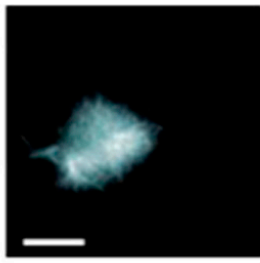

(a)

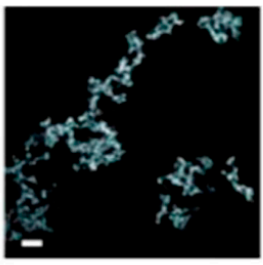

(b)

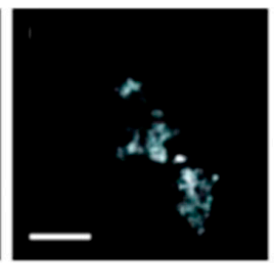

(c)

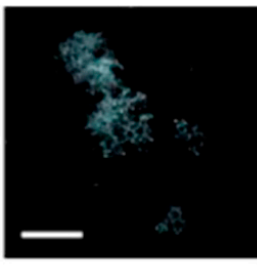

(d)

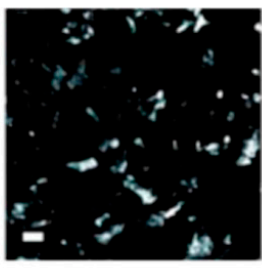

(e)

Figure 5. Intrinsic visible FL detected from five different amyloid systems: (a) $A \beta_{40}$, (b) $A \beta_{42}$, (c) $\mathrm{A} \beta_{33-42,}$ (d) K18 tau, (e) I59T. Excitation was at $450 \mathrm{~nm}$ wavelength and the fluorescence emission at $>488 \mathrm{~nm}$ was recorded. Scale bars are $20 \mu \mathrm{m}$. (Taken from [1]).

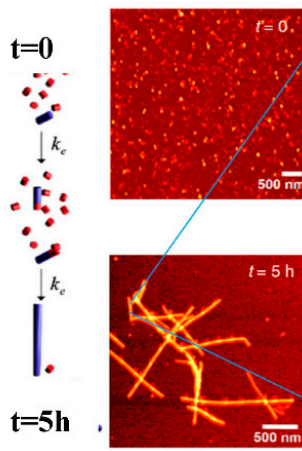

(a)

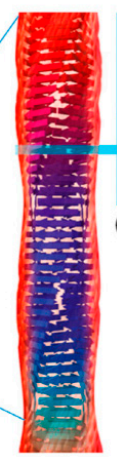

(b)

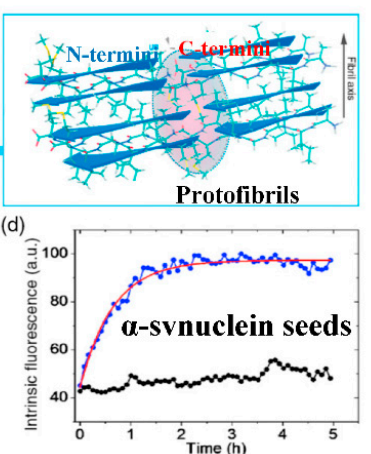

(c)

Figure 6. Intrinsic FL displays kinetics of $\alpha$-synuclein amyloid fibrils growth using a super high-resolution fluorescence microscopy tool. The FL measurements were performed with a diode laser emitting near $405 \mathrm{~nm}$ and signals were detected around $450 \mathrm{~nm}$. Short fibrillar seeds of $\alpha$-synuclein, schematically shown in blue, serve as the template from which fibril elongation proceeds through the addition of monomers, shown in red (a). The top panel to the right demonstrates atomic force microscopy (AFM) images of short fibril seeds at the start of the reaction, at $t=0$. Below is an image of the same sample at $\mathrm{t}=5 \mathrm{~h}$. Clearly, the elongation of the seeds into long amyloid fibrils has taken place. The structural model (b) of amyloid fibril shows that it consists of many ultrathin intertwined protofibrils. Zoomed-in region (c) indicated in (b) that exhibits the atomic arrangement between adjacent $\mathrm{N}$ - and $\mathrm{C}$-termini from these intertwined protofibrils. The applied FL microscopy technique allowed to observe the growth process of amyloid fibrils by recording in time intrinsic FL from the amyloid fibril (d). The blue points correspond to the elongation of $\alpha$-synuclein seeds in the presence of $\alpha$-synuclein monomer. The black points correspond to the addition of $\beta$-synuclein monomers to the $\alpha$-synuclein seed fibrils and confirm that the two species cannot "cross-seed" aggregation. (Taken from [85]).

Additional information on the FL phenomenon was assessed by a comparative study between amyloid fibrils grown from thirteen different proteins of both diseased- and non-diseased origin [98]. The FL spectra unexpectedly revealed two optical regions: a) FL in the visible range with the maximum at 430-450 $\mathrm{nm}$ (blue luminescence) under excitation 350-370 $\mathrm{nm}$ and b) near-infrared (NIR) FL emission 670-820 nm under excitation 620-780 nm (Figure 7). The blue FL enabled to examine and visualize amyloid aggregates and fibers in vitro without any staining technology. The results obtained for the visible region are completely consistent with all previously published data on FL in amyloid proteins of 
different compositions [83-87] and confirm the universality of this FL effect. The notable enhancement of FL intensity in fiber formation and the growing of amyloid fibers gave valuable information about amyloidosis kinetics.

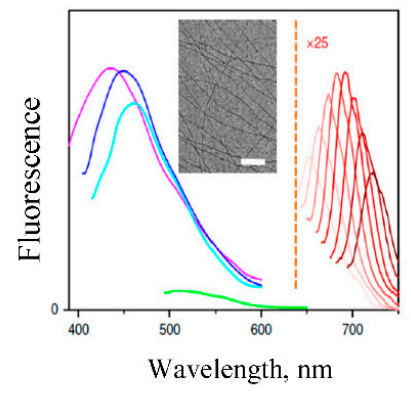

(a)

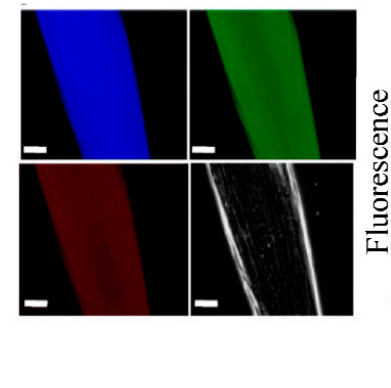

(b)

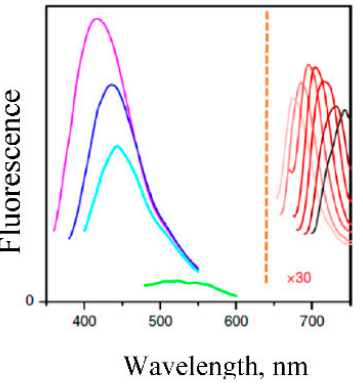

(c)

Figure 7. UV-visible-NIR FL of Het-s prion domain and $A \beta_{1-42}$ amyloid fibers. (a,c) FL of dispersions of Het-s prion domain (a) and $A \beta_{1-42} ;(\mathbf{c})$ amyloid fibers in buffer. Excitation wavelengths: $340 \mathrm{~nm}$ (pink), $360 \mathrm{~nm}$ (blue), $380 \mathrm{~nm}$ (cyan), and $440 \mathrm{~nm}$ (green), and from 600 to $670 \mathrm{~nm}$ (red color scale). A multiplying factor (either $\times 25$ or $\times 30$ ) has been applied to the NIR spectra to show both types of luminescence in the same graph. Inset in a: TEM image of Het-s prion domain. Scale bar, $100 \mathrm{~nm}$. (b) Fluorescence microscopy images with emission in the UV/Visible $(\lambda$ exc $=390 \pm 10 \mathrm{~nm}, \lambda \mathrm{em}=460 \pm 50 \mathrm{~nm}$ ) (top left), green $(\lambda$ exc $=475 \pm 10 \mathrm{~nm}, \lambda \mathrm{em}=530 \pm 50 \mathrm{~nm})($ top right $)$ and NIR $(\lambda \mathrm{exc}=620 \pm 60 \mathrm{~nm}, \lambda \mathrm{em}=$ $700 \pm 75 \mathrm{~nm}$, false red color) (bottom left). (Taken from [98]).

This study disclosed some new and promising generic features of this biophotonic FL phenomenon [98]. The first one is a wavelength-dependent mechanism in which the spectral position of the FL peak is displaced upon variations in the exciting wavelengths. The second feature is a new observation of the FL signal in the infrared (NIR) region. The NIR FL photons we found are within the "biological window" within the wavelength $>800 \mathrm{~nm}$, which transforms this FL effect from a curious scientific phenomenon to an effective tool of investigation of amyloid diseases in vivo. In [98], the authors demonstrated that amyloid fibrils of Alzheimer's disease in a living mouse model exhibit a strong NIR signal that can be detected by 3D imaging, and which also provides non-invasive and real-time 2D NIR imaging on live animals and offers prospects for diagnostics of neurodegenerative diseases.

To explain the nature of such intrinsic FL in $\beta$-sheet rich amyloid structures, molecular dynamics simulations combined with FL spectroscopy were performed [87]. It was demonstrated that these intrinsically FL protein fibrils are permissive to proton transfer across hydrogen bonds which can lower electron excitation energies and thereby decrease the likelihood of energy dissipation associated with conventional hydrogen bonds. This leads to situations in which the proton can be found on either the $\mathrm{N}$ - or the C-terminus and thus to the creation of a double-well ground state potential that is likely to have a consequences for both the absorption and emission characteristics of the system [87].

\section{Fluorescence Effects in Bioinspired Nanostructures}

In the previous chapter, we considered a new biophotonic phenomenon of visible intrinsic FL found in diverse human disease-related amyloid nanostructures of different origins, compositions, and complexities. All these biological structures share a similar morphology of fibrillary networks folded into the same secondary $\beta$-sheet architecture from native soluble $\alpha$-helical conformations.

In this chapter, we show that synthetic biomolecules, self-assembled into solid-state nanostructures, mimic their biological counterparts. They are also folded into two different biomolecular configurations, either helix-like or $\beta$-sheet states and exhibit, in these two states, different morphologies and completely different fold-sensitive physical properties. We have shown that in helix-like structures, piezoelectric [99], nonlinear optical [100] and electrooptical [101] effects are observed. All $\beta$-sheet 
structures are self-organized into an amyloid-like fibrillary morphology and demonstrate visible FL [2,89-92].

\subsection{Native and Thermally Induced Peptide Nanostructures: Morphologies and Secondary Structures}

This chapter is focused on a deep modification of ultrashort peptide nanostructures self-assembled from aromatic di-(FF), tri-phenylalanine (FFF) and aliphatic di-leucine (LL) chemically synthesized monomer biomolecules. We studied, in these structures, the dynamics of variations in physical properties under helix-like $\rightarrow \beta$-sheet transformation, which can be used a model for amyloid transformation in a human organism.

To overcome the energy barrier between these two basic states $[77,102]$ the fundamental interconversion of biological origin was performed at elevated temperatures. It has been found that $\alpha$-helical $\rightarrow \beta$-sheet transformation is irreversible and can be studied by gradual, step-by-step temperature variations in the range of $25-160{ }^{\circ} \mathrm{C}$ that allowed us to observe the critical stages of this biological refolding, appearance and modification of physical properties.

At room temperature, the di- and tripeptide linear aromatic diphenylalanine (FF), aliphatic dileucine (LL) and triphenylalanine (FFF) biomolecules were self-assembled into various morphological shapes by colloidal techniques. In aqueous solution, linear FF and LL biomolecules acquired hollow peptide nanotube morphologies (FF-PNT, LL-PNT), whereas FFF biomolecules adopted a micro-nanosphere morphology in a chloroform solvent (Figure 8a-c) [2] and a tape morphology in aqueous solution [90].
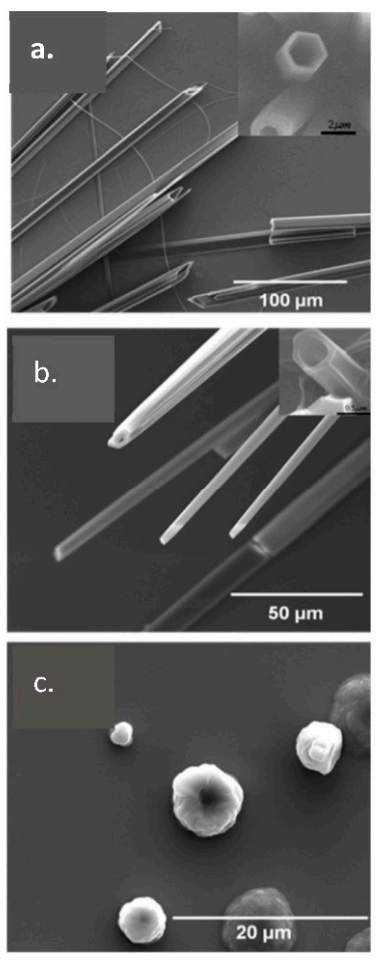
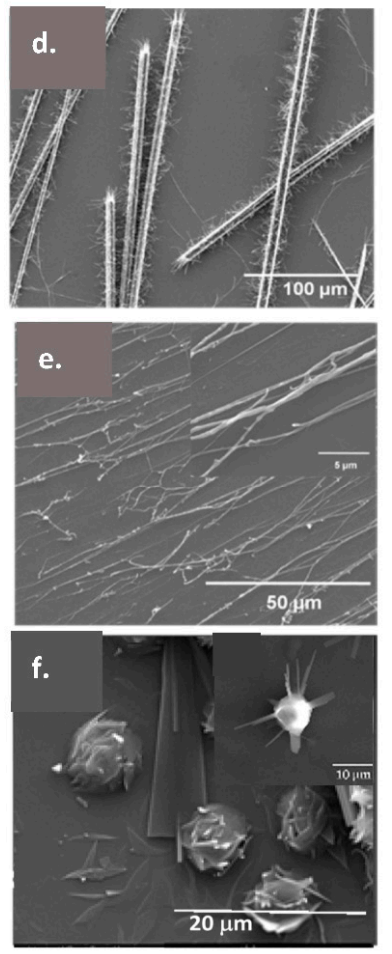
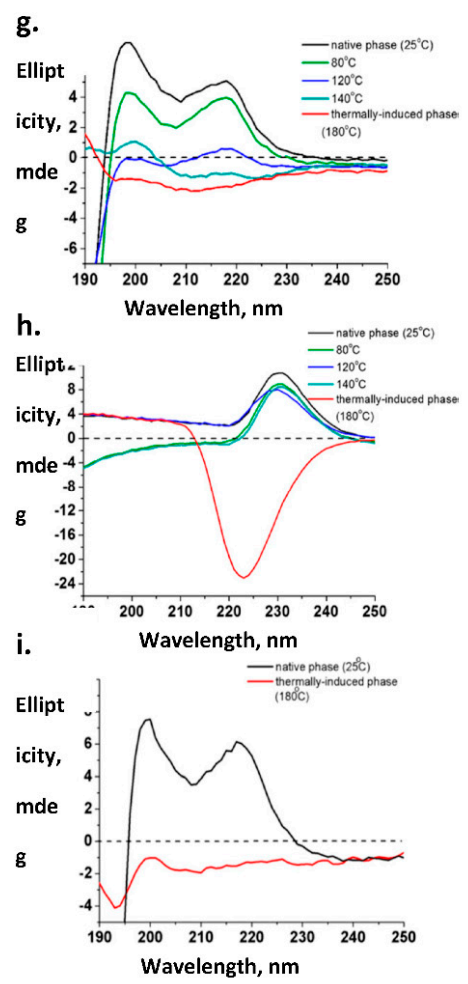

Figure 8. Morphology (a-f) and secondary structure (g-i) of peptide micro-nanostructures of different origin in native, helix-like state $(\mathbf{a}-\mathbf{c})$ and thermally induced $\beta$-sheet state $(\mathbf{d}-\mathbf{f})$. (a-c) SEM images of (a) native, $\alpha$-helical diphenylalanine (FF)-peptide nanotubes; (b) aliphatic dileucine (LL)-nanotubes and (c) triphenylalanine (FFF)-microspheres, the inset images at (a,b) show regular cross-sections of the FF and LL nanotubes; (d-f) SEM images of thermally induced $\beta$-sheet FF and LL ultrathin peptide nanofibers and FFF peptide spheres, the inset (f) shows peptide fibers grown from a single FFF sphere. (g-i) Peptide secondary structure evolution of FF, LL and FFF nanostructures under thermally mediated transformation, examined by circular dichroism method. (Taken from [2]). 
Both dipeptide FF and LL nanotubes have identical open-end tubular shapes. The length of the PNTs can reach hundreds of micrometers $(>300 \mu \mathrm{m})$ and the diameter is about $\sim 2 \mu \mathrm{m}$ (Figure $8 \mathrm{a}-\mathrm{c}$ ). They consist of basic monomer amino acids, phenylalanine and leucine, and are stabilized by their hydrophobic side groups [103]. The inset images (Figure 8a,b) show cross-sections of single native PNTs, hexagonal for FF PNT, and orthorhombic for LL-PNT, enabling their assignment to different crystallographic classes [104]. They are related to asymmetric structures where native FF-PNTs have hexagonal symmetry $\mathrm{P} 6_{1}$ and LL-PNTs have orthorhombic $\mathrm{P} 2_{1} 2_{1} 2_{1}$ symmetry [91]. The intrinsic asymmetry of the native phase of dipeptides and tripeptides is the basis for the exploration of fundamental physical phenomena described by tensors of the third rank, such as piezoelectric [99], nonlinear optical [100] and linear electrooptical [101] effects. In a non-polar chloroform solvent tripeptide FFF monomer is assembled into sphere-like structures with an average diameter of 2-6 $\mu \mathrm{m}$ (Figure 8c). A similar spherical structure was self-assembled from the same FFF biomolecules of Boc-FFF [105]. The FFF monomer can also adopt a tape-like morphology in a polar aqueous solution [106]. These FFF planar structures can reach large $X-Y$ dimensions of several dozens of micrometers [90].

When subjected to thermal treatment, all studied diverse peptide structures pass a similar dramatic modification (Figure 8d-f). Regardless of their chemical composition and native shapes, all of them are transformed to a fibrillary morphology $[2,92,107]$. These ultrathin fibers can reach dozens of $\sim(1-20)$ micrometer in length and are 20-200 nm thick. Here, we show that their morphology, peptide secondary structure, and basic properties are similar to biological amyloid structures.

A key factor defining basic biological $[108,109]$ and physical properties $[2,91,92,107]$ in peptide/protein structures is their secondary structure, helix-like composition or $\beta$-sheet. To examine the secondary structure of the peptide ensembles in their native and thermally induced states, we applied circular dichroism tool (CD) [110] and Fourier-transform infrared-spectroscopy (FTIR) methods [111]. $\mathrm{CD}$ and FTIR are standard methods for monitoring the conformation kinetics of bioinspired and biological materials [112] and especially for revealing secondary-structural changes such as the detection of $\beta$-sheet structures that accompany the formation of amyloid fibrils $[102,109,113]$. The CD spectra (Figure 8g-i) were measured for all self-assembled FF, LL, FFF peptide nanostructures vs. temperature. For the native phase, the CD spectrum of FF-PNT (Figure 8a) exhibits a positive band with two broad peaks of ellipticity (Figure 8g). The first maximum of ellipticity is $\Delta \varepsilon=\sim 5 \mathrm{mdeg}$ at $\sim 198 \mathrm{~nm}$. The second maximum of ellipticity is at $\sim 218 \mathrm{~nm}(\Delta \varepsilon=\sim 7.5 \mathrm{mdeg})$. As the temperature of the preliminary heat treatment is increased, the ellipticity gradually decreases, and at $\mathrm{T} \sim 140{ }^{\circ} \mathrm{C}$, it crosses a zero line and becomes negative. Finally, at $180^{\circ} \mathrm{C}$, a broad negative peak $(\Delta \varepsilon=-2.2 \mathrm{mdeg})$ at $210 \mathrm{~nm}$ is observed. Such a change in the circular dichroism reflects a major variation in the structural rearrangement of FF peptide ensembles and the creation of a new secondary structure. In the native phase of LL-PNTs (Figure 8 b), the CD spectrum shows a single positive Gaussian-shaped band (Figure $8 \mathrm{~h}$ ) with maximum ellipticity of $\Delta \varepsilon \sim 11$ mdeg at $230 \mathrm{~nm}$, which corresponds to amide $\mathrm{n}-\pi^{*}$ transition [114]. This electronic transition typically lies between $210-230 \mathrm{~nm}$, and is the lowest energy transition in the amide group. As the temperature is raised, the measured ellipticity of the peak decreases at $\sim 140{ }^{\circ} \mathrm{C}$, it changes its sign and is transformed at $180^{\circ} \mathrm{C}$ into a strong, irreversible negative peak $(\Delta \varepsilon=-23 \mathrm{mdeg})$ at $223 \mathrm{~nm}$. The inversion of the CD spectrum of LL-PNT at an elevated temperature is similar to the behavior of FF-PNT.

The CD peaks of tri-peptide nanospheres, FFF-PNS, at room temperature in the native phase, exhibit two positive bands with maximum ellipticity at $\sim 199 \mathrm{~nm}$ and $\sim 217 \mathrm{~nm}$ and minimum ellipticity at $\sim 209 \mathrm{~nm}$ (Figure $8 \mathrm{i}$ ). These values are very close to those found in the native FF-PNT (Figure 8g,i). Positive CD bands, observed in the native phase of FF, LL and FFF nanostructures, were also found in proteins, such as Gene5 and avidin [114], and many phenylalanine-based nanostructures [115,116]. After heating to elevated temperatures, the CD spectrum of the formed FFF fibers becomes negative. This is completely consistent with the temperature behavior of FF and LL nanostructures when they undergo thermally induced conversion at $\mathrm{T}=140-180^{\circ} \mathrm{C}$. 
Peptide/protein folding and unfolding processes are the subject of detailed studies where the basic information on their secondary structure is found from circular dichroism (CD) optical spectroscopy data. This method is applied for monitoring biological transformations and studies of self-assembled amyloid-diseased and non-diseased peptide/protein structures [117,118]. To characterize peptide/protein secondary structures, the CD tool uses the "fingerprinting" in the UV region of 190-250 nm. Specific CD spectral minima are observed for $\alpha$ helical structures in the absorption spectra around 208 and $222 \mathrm{~nm}$, and for $\beta$-sheets in a single peak at 216-220 nm. A comparison of the CD obtained experimental data (Figure $8 \mathrm{~g}-\mathrm{i}$ ) with CD spectral fingerprints $[110,112-114,117]$ enabled us to ascribe the native, self-assembled FF, LL nanotubes and FFF nanospheres to the helix-like state (Figure $8 \mathrm{a}-\mathrm{c}$ ) and thermally induced fibrillary structures to the $\beta$-sheet one (Figure $8 \mathrm{~d}-\mathrm{f}$ ).

We show in the next chapter that bioinspired amyloidogenic $\beta$-sheet fibrillary peptide structures acquire absolutely new physical property of visible FL. Found FL in these man-made structures exhibits similar excitation and photon emission optical spectra to those found in amyloid structures.

\subsection{Fluorescence Phenomena in Native and Thermally Induced Peptide Nanostructures}

\subsubsection{Fluorescence Effect in Native Helix-Like Bioinspired Peptide Nanostructures}

The original electronic structure of elementary biomolecules (amino acids, peptides, proteins) does not exhibit any intrinsic visible photon emission except the unique FL protein of the jellyfish, Aequorea victoria, the green FL protein (GFP). It displays strong optical absorbance in a narrow blue region followed by green FL with a quantum yield close to 0.8-0.9 [119]. In the UV region, FL was found in a few limited aromatic amino acids such as tryptophan, demonstrating FL at $350 \mathrm{~nm}$, as well as the amino acids tyrosine and phenylalanine with characteristic FL at $303 \mathrm{~nm}$ and $282 \mathrm{~nm}$, respectively [120]. Some of these biomolecules are used for direct monitoring of basic biological processes both in vivo and in vitro in cells and tissues in biomedical research [19].

In this chapter, we review the FL phenomena at the level of self-assembled peptide structures of aromatic and aliphatic origin related to two different conformations, helix-like (Figure 9) and $\beta$-sheet (Figure 10). Optical absorption (OA) and FL excitation spectra (FLE) are the direct methods for studying the electron energy spectrum of matter and its FL properties
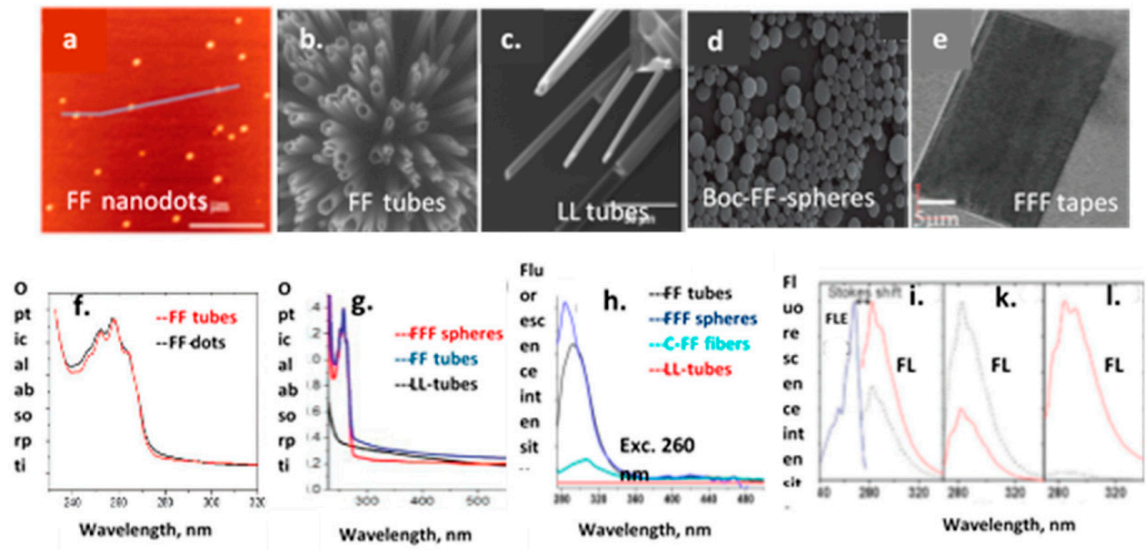

Figure 9. Optical absorbance and fluorescence phenomena in native $\alpha$-helical ultrashort peptide structures. (a) Atomic force microscopy image and (b-e) SEM images of (a) FF nanodots, (b) FF nanotubes, (c) LL nanotubes, (d) Boc-FF-spheres, (e) FFF tapes. (f) Optical absorption of FF tubes and FF dots; (g) Optical absorption of FFF spheres, FF tubes, LL tubes; (h) Fluorescence spectra of FF tubes, FFF spheres, Cyclic-FF fibers, LL tubes; (i-1) Fluorescence (FLE) excitation spectra, (for FL $285 \mathrm{~nm}$ ); FLE and FL spectra of peptide Boc-FF spheres of $4 \mathrm{mg} \mathrm{mL}^{-1}$ (red solid lines) and $1 \mathrm{mg} \mathrm{mL}^{-1}$ (black dashed lines) concentrations at (i) two excitation wavelengths of 270 and $255 \mathrm{~nm}$; (k) for Boc-FF spheres and (1) Boc-FF monomer respectively); (i)The FLE spectrum and the FL Stokes shift (15 nm). (Taken from $[2,91,121])$. 
Figure 9f,g,i,k depict the normalized OA and FL spectra of FF, FFF, Boc-FF, Cyc-FF nanostructures and the Boc-FF-monomer. Peptide nanostructures of ultrashort aromatic di-phenylalanine (FF), tri-phenylalanine (FFF), and aliphatic di-leucine (LL) are optically different and recognized both in OA and FL spectra according to their core phenylalanine and leucine amino acids. It can be seen that the tested five aromatic amino acid-based nanostructures (FF dots, FF tubes, FFF spheres, Cyc-FF-fibers, Boc-FF spheres and Boc-FF monomer) share similar OA spectra in the UV region, where a strong OA peak appears around $\sim 260 \mathrm{~nm}$. Analysis of these spectra shows that this peak is followed by two satellite sub-peaks, located at $265 \mathrm{~nm}(4.68 \mathrm{eV})$ and $253 \mathrm{~nm}(4.90 \mathrm{eV})$, reflecting its fine structure. The energy intervals between two neighboring peaks are equal to $0.1-0.11 \mathrm{eV}$, which is ascribed to benzoic rings of phenylalanine, F-amino acid [120]. Our data also show that aliphatic leucine-based LL di-peptide native nanotubes do not demonstrate OA and/or FL effects in this UV spectral interval. Studies of OA in leucine amino acid-based films show that OA's far UV region is observed at 120-190 nm only [122].

FL spectra in the UV region of F-aromatic resides exhibit very small Stokes shifts $\sim 15 \mathrm{~nm}$ and narrow resonant-like FL peaks around $290 \mathrm{~nm}$ with $\lambda \sim 20-30 \mathrm{~nm}$ for all aromatic FF, FFF, Boc-FF, Cyclic-FF-peptide structures (monomers, nanodots, tubes, fibers, spheres, tapes) regardless of their composition, morphology or structural architecture. These FL parameters are similar to organic dyes. The most important feature of these optical properties is insensitivity of FL spectra in the UV region to peptide secondary conformation ( $\alpha$-helices or $\beta$-sheets), demonstrating, in the UV region, the same OA and FL optical signatures in the range of $250-290 \mathrm{~nm}$ for all phenylalanine-based structures (Figures 9 and 10). No visible FL is observed in helix-like native peptide structures.

Thus, our experimental data (Figure 9) show that aromatic peptide nanostructures demonstrate the optical properties of OA and FL in the UV region around $260-290 \mathrm{~nm}$. They are not influenced by the variation in morphology or peptide secondary structure (helix-like or $\beta$-sheet) and can be presented as the invariant fold-insensitive optical signature of phenylalanine residues composing aromatic peptide nanostructures.

\subsubsection{Visible Fl in Bioinspired Amyloidogenic B-Sheet Structures}

In this part of the chapter, we consider the completely different biophotonic phenomenon of visible FL. This photonic effect is observed in amyloid [85-88,98] and amyloidogenic [2,3,89-92,122] structures irrespective on their amino acid/peptide/protein composition. The physical origin of the revealed visible FL is a specific biomolecular arrangement of fundamental biological $\beta$-sheet folding. Such a supramolecular configuration creates a new electronic structure and electronic transitions, allowing the generation of visible FL photons both for highly complicated disease-related amyloid proteins and ultrashort chemically synthesized di- and tri-peptides self-assembled into $\beta$-sheet fibrillary structures.

Moreover, in ultrathin human brain $\beta$-sheet nanowires, the product of a long crystallization process from soluble $\alpha$-helical amyloid proteins can be found [102,109]. In the bioinspired materials, we observed similar fibrillary structures grown under the thermally induced transformation of the native helix-like state to the $\beta$-sheet one. These $\beta$-sheet amyloidogenic fibers deeply change original non-fluorescent optical properties and lead to the appearance in the $\beta$-sheet state of a new fold-sensitive effect of visible FL phenomenon in the region 400-700 $\mathrm{nm}$ [2,3,89-93].

Figure 10 accumulates basic experimental data on the helix-like $\rightarrow \beta$-sheet transition and FL phenomena in bioinspired materials. It demonstrates the fine development of this thermally mediated interconversion of biological origin. We found, for bioinspired nanostructures, that this fundamental process is an irreversible process that allowed us to study it at all stages and all properties in detail. The first step in these studies is a direct observation of morphological changes in a single FF nanotube and its dynamic transformation into ultrathin nanofiber by the use of different high-resolution microscopy tools (Figure 10a,b). The gradual closing of the central hole under electron beam heating of the FF-PNT and its conversion to the fiber structure is followed by a clear variation in the cross-section when the pronounced hexagon is transformed into a shapeless structure preceding its final crystallization to the 
fibrillary shape (Figure 10a,b). This "smashing" provides direct evidence of the intermediate stage of this structural refolding between native, diverse state/morphology (tube, spheres, tape, and more) and final fiber morphology. The fibrillary amyloid-like structures are adopted under heat treatment by many different peptide structures: LL tubes, FFF spheres, FFF tapes (Figures 8 and 10b,g). The same fiber morphology formation is also observed in vapor-deposited FF structures [4] demonstrating the fibrillization process directly from FF peptide monomers (Figure 10k,l).
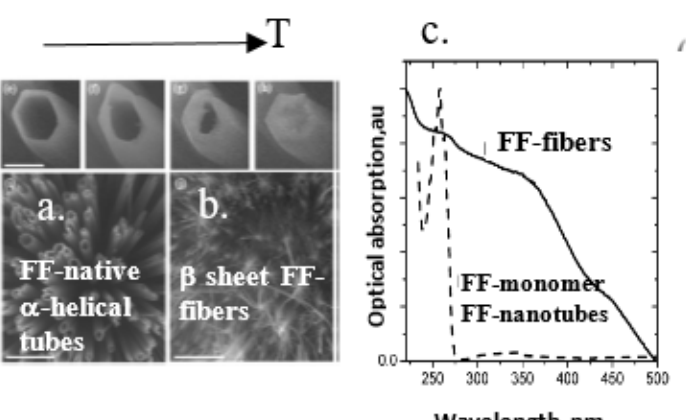

Wavelength, $\mathrm{nm}$
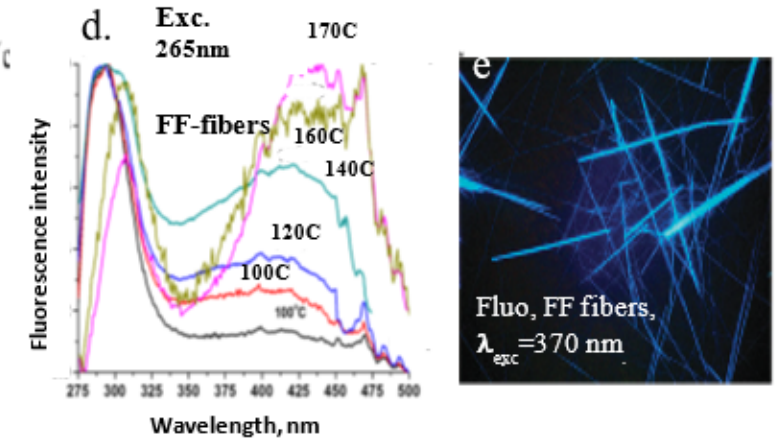

Wavelength, $\mathrm{nm}$
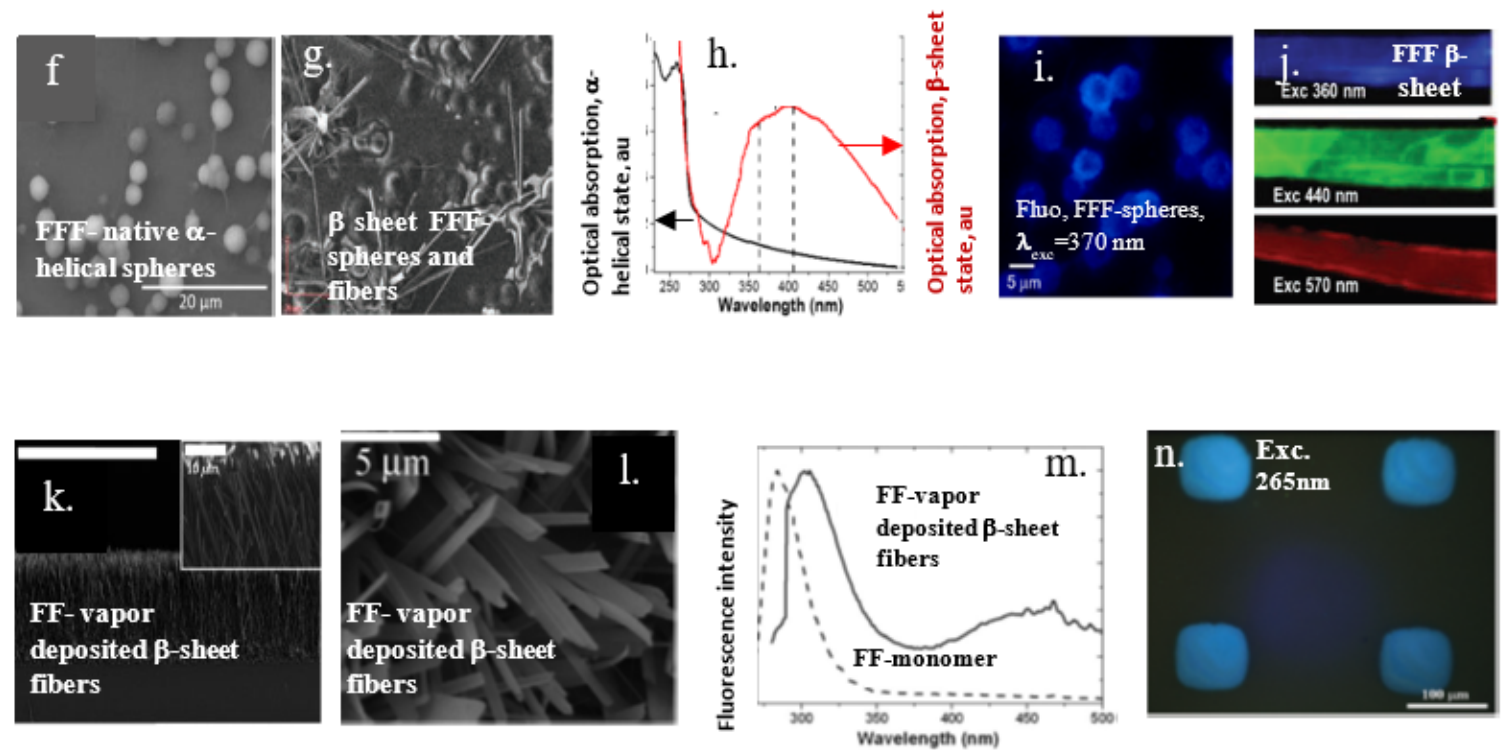

Figure 10. Visible fluorescence, optical absorption and morphology transformation in thermally refolded helix-like $\rightarrow$. $\beta$-sheet amyloidogenic peptide nanostructures of different origin. $(\mathbf{a}, \mathbf{b}, \mathbf{f}, \mathbf{g})$ Morphological transformation (SEM images) from helix-like FF nanotubes and FFF spheres to $\beta$-sheet fibrillary structures; (k,l) vapor-deposited FF fiber structures (SEM images). Modification of optical absorption spectra under helix-like $\rightarrow \beta$-sheet transformation in: (c) FF nanotubes $\rightarrow$ FF fibers; (h) FFF spheres $\rightarrow$ FFF fibers; Visible FL in thermally induced $\beta$-sheet fibrillary structures of: (d) The dynamics of the FL effect; (e) FF fibers; (i) FFF spheres/fibers; (j) FFF tapes; (m) vapor-deposited fiber structures. (n) FF vapor-deposited patterned array. (Taken from [2,4,89-91]).

This unique solid-state reconstruction of biological origin is followed by the transformation of the peptide secondary structure from native, $\alpha$-helical to $\beta$-sheet, which is another common property of all types of the studied peptide structures (Figure 8). It should be reminded that such a transformation starting from the native protein state and ending by fibrilization is also observed in all biological disease-related amyloid peptides/proteins [102,109] and bioinspired materials [2,90] and considered as a basic property of biological and bioinspired peptides/proteins that have undergone $\alpha$-helical $\rightarrow \beta$-sheet refolding [102]. The morphological rebuilding of ultrashort peptide structures we found is consistent with that proposed by Dobson's group [102,109], "amyloid approach", which widened the class of 
amyloid disease-related proteins to an unlimited number of natural and bioinspired peptides and proteins that have undergone to universal $\alpha$-helical to $\beta$-sheet transformation.

Full reconstruction of peptide/protein self-assembled structural organization leads to a profound variation in morphology, structural symmetry, bimolecular arrangement (secondary structure), and more. The experimental data (Figure 10), displaying the described transformations for peptide structures of different compositions and morphologies also show that the found macroscopic and microscopic structural changes are also accompanied by a deep modification of the electronic systems of these supramolecular structures. All studied aromatic peptide structures exhibit two different types of OA and FL. The first one is observed for aromatic structures in the UV region of $260-310 \mathrm{~nm}$ (Figure $9 \mathrm{c}, \mathrm{d}, \mathrm{h}, \mathrm{m}$ ). The narrow UV spectra of OA and FL are related to aromatic rings. They are insensitive to morphology, symmetry, and are invariant in both helix-like (Figure 9 ) and $\beta$-sheet (Figure 10) states, making them fold-insensitive optical properties.

However, the second type of OA and FL is completely different. The described biological transition from native helix-like to $\beta$-sheet structure creates a new broadband OA and FL, covering the entire visible region, with no isolated spectral lines occupying, for all biological and bioinspired materials, a strictly definited spectral interval of $\sim 400-700 \mathrm{~nm}$ (Figure $10 \mathrm{c}-\mathrm{e}, \mathrm{h}-\mathrm{j}, \mathrm{m}, \mathrm{n}$ ). These generated fold-sensitive optical properties are observed in the $\beta$-sheet structures only from any amyloid or amyloidogenic peptide/protein structures. The general concept of supramolecular structures considers the peptide/protein structure as two subsystems of biomolecular organization where one comprises peptide/protein biomolecules and another one comprises the noncovalent hydrogen bonds binding these biomolecules into stable and steady structures. The discussed fundamental refolding helix-like $\rightarrow \beta$-sheet phenomenon does not change the basic biomolecules' composition and their electronic structure, but it does reconstruct their hydrogen bonds. In fibrillary $\beta$-sheet structures, the non-covalent hydrogen bonds interconnect the $\beta$-strands into a $\beta$-sheet configuration. Molecular dynamic simulations showed that these rebuildt $\mathrm{H}$-bonds allow proton transfer between the $\mathrm{N}$ - or the C-terminus of peptides/proteins in a cross $\beta$-sheet fibrillary structure, creating a double-well ground state potential which results in optical absorption in the near UV range and FL in the visible range [87].

A great advantage of the applied thermally induced method is a unique chance to observe both fine details of the of $\alpha$-helix $\rightarrow \beta$-sheet transformation (morphology and secondary structure) (Figures 8 and 10a,b) and the dynamics of the FL effect during this process (Figure 10d) [91]. The demonstrated set of FL optical spectra for refolded FF nanotubes (Figure 10a,b,d) was recorded for a few temperature points in the interval $100-170{ }^{\circ} \mathrm{C}$. Two different optical regions of FL are observed. The first type is FL UV spectra, which are related to aromatic rings. They were described in the previous chapter and detected in both helix-like and $\beta$-sheet states, showing temperature-independent behavior. Another part of the FL spectra is revealed in the visible region. The visible FL intensity rises with the temperature which could be related to the increase in $\beta$-sheet concentration under refolding. This assumption is consistent with the found ultrathin amyloid-like fibers grown in FF nanotubes during heating $[2,92]$.

FL exhibits a very broad and continuous spectrum in a vast range of $400-700 \mathrm{~nm}$, with its maximum value in a blue region at $\sim 450 \mathrm{~nm}$ (Figure 10d). Our experiments demonstrate that the FL spectrum is excitation dependent [2,3,90-93]. Color FL images, throughout the whole visible spectrum (Figure 10e,i,j,n), as well as tunable FL in various peptide structures such as FF, FFF, LL fibers, spheres, tapes, nanodots, and more obtained for different FL excitation wavelengths, display promising photonic applications in biophotonic technology, bioimaging and bioptical chips [2,3,89,91-93]. In the next chapter, we describe visible FL bionanodots of different origin developed for various biomedical technologies $[3,93]$.

\subsection{Fluorescence in Pegylated Films and Fibers}

The de novo design of short peptides and their polymeric derivatives has been recently identified as an operative approach for the development of innovative optoelectronics tools. Polymer-peptide 
based materials allow for the combination of synergistical functionality (biocompatibility, low cost, easy chemical modification) and the capability of peptides to recognize the cheapness and responsiveness of polymers. Among the synthetic polymers, poly(ethylene glycol) (PEG) is considered a good standard, and several PEG-based materials have been approved by the Food and Drug Administration (FDA) for different biomedical applications. Having a non-immunogenic and biocompatible profile, PEG bioconjugation (also referred to as PEGylation) finds applications in drug delivery [123], micelles, and liposome formulation [124], tissue engineering [125] and in pharmacokinetic profiles optimization [126]. Several hetero-peptides such as poly ( $\gamma$-benzyl-l-aspartate) [127], [(AG)3EG]10 [128], DGRFFF [129], and (LELL) [130] have been PEGylated efficiently and fully characterized in their self-assembled states. An additional advantage of PEGylation is the possibility to modulate the water solubility and the structural organization of the final material.

Recently, several poly aromatic peptides such as diphenylalanine (F2), tetra-phenylalanine (F4), and hexaphenylalanine (F6) were derivatized with PEG moieties and the optoelectronic properties of these self-assembled materials were studied. Self-assembled aggregates of these aromatic peptides share a common structural organization: they assemble into fibrillary nanostructures with a high content in $\beta$-sheet structures. Moreover, it was observed that the PEG length plays an important role both in the morphology and optical properties of the resulting self-assembled nanomaterial. This result is strictly related to the hydrophobic/hydrophilic ratio in the polymer/peptide.

The simplest PEGylated peptides described up to now are PEG2-FF and PEG6-FF (Figure 11A) [130]. In these peptides, the F2 homodimer is derivatized at its N-terminus by two (PEG2) or six (PEG6) ethoxylic moieties. Due to the PEG moiety, both the peptides present a higher water solubility and a higher critical aggregation concentration (CAC $=3.62$ and $19.5 \mathrm{mg} / \mathrm{mL}$ for PEG2-FF and PEG6-FF, respectively) compared to the FF dipeptide alone (CAC $=0.33 \mathrm{mg} / \mathrm{mL}$ ). Above their CAC values, PEG2-FF generates ultrastructures as a result of conglomerates of shorter untwisted fibers, while PEG6-FF self-assembles into longer twisted fibers. Secondary structural characterization performed by CD, FTIR, and spectroscopic assays highlighted the formation of amyloid-like structures rich in $\beta$-sheets. According to the electron delocalization via hydrogen bonds hypothesis, these structural features confer to the peptide solutions FL properties in the blue region of the visible spectrum $\left(\lambda_{\mathrm{em}}=420\right.$ and $460 \mathrm{~nm}$ for PEG2-FF and PEG6-FF). Both the peptides also exhibited a Red Edge Excitation Shift (REES) phenomenon, suggesting a high degree of flexibility at the molecular level in the PEGylated aggregates.

Other optically active polymer/peptide materials were prepared by using different PEGylated F4 and F6 building blocks. An example of the F4 derivative was reported by Castelletto et al. in 2009 [131]. The synthesis and the self-assembling behavior of PEG5000-F4 was reported. As expected, the self-aggregation of monomers occurs via hydrophobic aromatic interactions above a CAC value of $0.095 \mathrm{wt} \%$. The resulting fibrillary structures exhibited an FL emission peak, proportional to the concentration, around $560 \mathrm{~nm}$ upon excitation of the PEG5000-F4 aqueous solution at $440 \mathrm{~nm}$.

Successively, Diaferia et al. described a series of PEGylated hexaphenylalanine conjugates (Figure 11B) in which the N-terminus of the aromatic framework was derivatized with PEG chains of different lengths (PEG8, PEG12, PEG18 and PEG24) [94,95]. The first of the series, PEG8-F6, was synthesized by sequentially coupling four units of amino-carboxy-PEG2 blocks, whereas the other three derivatives (PEG12, PEG18, and PEG24) were obtained by coupling two, three or four units of amino-carboxy-PEG6 blocks. This synthetic approach produces polymer/peptide adducts with a certain number of extra amide bonds in the PEG moiety. These amide bonds could provide additional intermolecular hydrogen bonds in the supramolecular architecture. All the peptides were found able to self-aggregate into aqueous solution as a consequence of a hydrophobic collapse phenomenon induced by dilution in water of a stock peptide solution $(100 \mathrm{mg} / \mathrm{mL})$ prepared in 1,1,1,1,3,3,3-hexafluoropropanol (HFIP). All the hexaphenylalanine analogues self-assemble at lower CAC values $(10.1,60.9,20.4$, and $13.9 \mu \mathrm{g} / \mathrm{mL}$ for the PEG18, PEG12, PEG18, and PEG24, respectively) with respect to the PEGylated-F2 and -F4 building blocks. This result can be explained as a direct 
consequence of the extension of the aromatic portion and the formation of additional intramolecular hydrogen bonds between the amide bonds in the PEG chain. According to the other shorter PEGylated peptides reported above, F6-derivatives also self-organize into fibrillary nanostructures of different lengths with a structural organization dominated by $\beta$-sheets with an antiparallel orientation of $\beta$-strands. However, Wide-Angle X-Ray Scattering (WAXS), both in transmission and reflection (GIWAXS) mode, highlighted a certain decrease in the fiber order along the series PEG8 > PEG12 > PEG18 > PEG24.
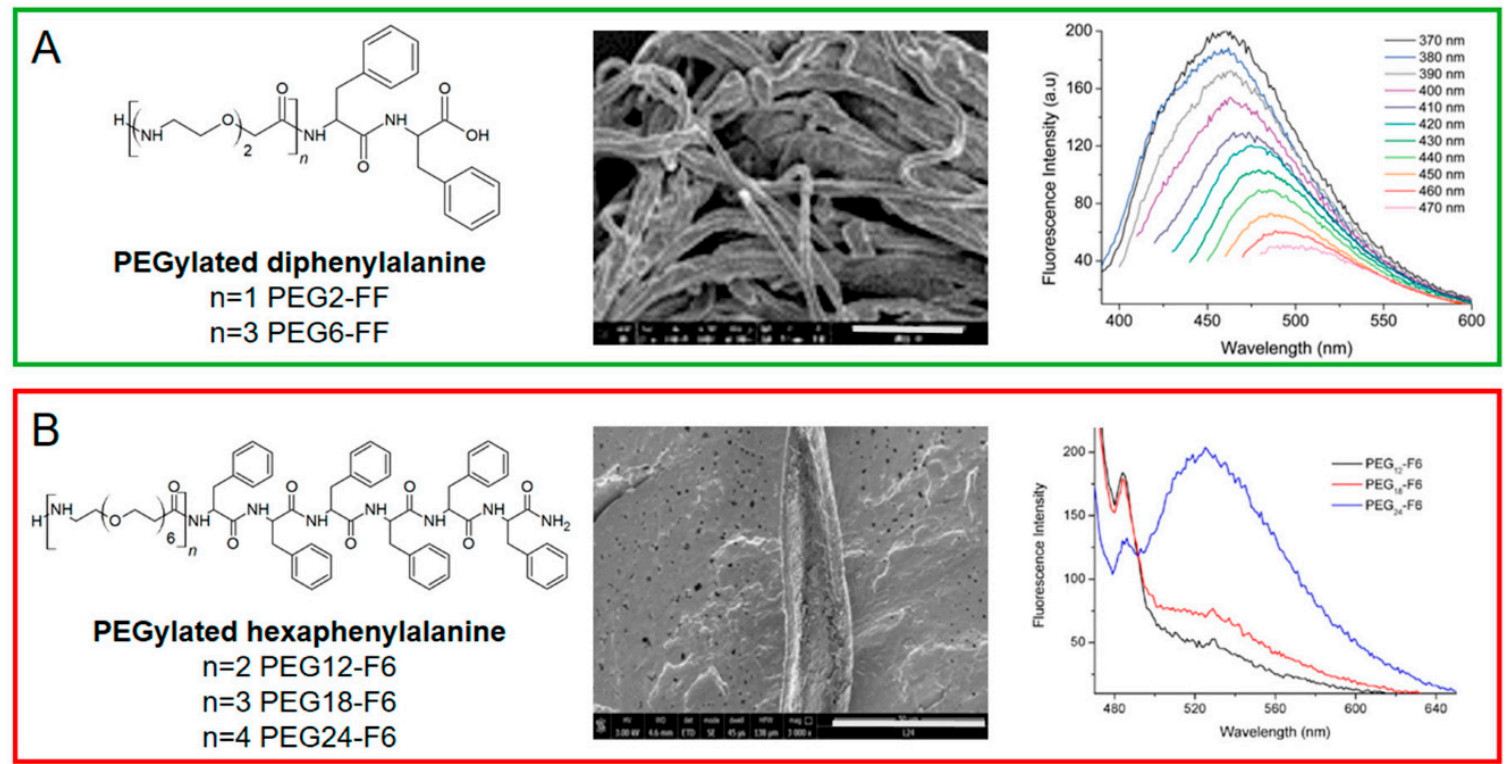

Figure 11. (A) Schematic presentation of poly(ethylene glycol) (PEG)2-FF and PEG6-FF, SEM image (scale bar of $5 \mu \mathrm{m}$ ), and fluorescence spectra for Peg6-FF aggregates in water solution at $70 \mathrm{mg} / \mathrm{mL}$. (B) Schematic presentation of PEG12-F6, PEG18-F6 and PEG24-F6, SEM microphoto of PEG24-F6 aggregates (scale bar of $50 \mu \mathrm{m}$ ), and fluorescence emission spectra of PEGylated-F6 derivatives in water at $10 \mathrm{mg} / \mathrm{mL}$. (Taken from [130]).

The FL study performed on PEG8-F6 aggregates (at $10 \mathrm{mg} / \mathrm{mL}$ ) highlighted a blue photoluminescence emission (between $420-460 \mathrm{~nm}$, exciting the sample both at 370 and $410 \mathrm{~nm}$ ). This behavior was maintained for the sample in the solid state. PEG8-F6-based nanostructures were able to transfer their intrinsic fluorescence via Föster resonance electron transfer (FRET) to an acceptor dye (4-chloro-7-nitrobenzofurazan) spatially close to them [132]. In this way, the original emission peak can be red shifted from the blue region $\left(\lambda_{\mathrm{em}}=460 \mathrm{~nm}\right)$ to the green and red regions of the visible spectrum (broad emission peak centered at $530 \mathrm{~nm}$ ). This evidence could enable new and promising applications for peptide biomaterials encapsulating dye in the bioimaging field for the detection of the aggregation process of endogenous proteins, which, in their physiopathological form, adopt a cross- $\beta$ structural motif.

Analogously to PEG8-F6 aggregates, PEG12-F6, PEG18-F6, and PEG24-F6 also exhibited optoelectronic properties in water in the blue visible region. Beyond the emission peak around $460 \mathrm{~nm}$, the fluorescence spectrum of PEG24-F6 is also characterized by a peak at $530 \mathrm{~nm}$, detectable upon excitation of the sample at $\lambda_{\text {exc }}=460 \mathrm{~nm}$ (Figure 11B). On the contrary, this emission peak appears to be absent or very weak for PEG12-F6 and PEG18-F6 analogs (Figure 11B). The same behavior was also observed for the three samples in the solid state. The optoelectronic properties of PEG24-F6 were explained by the formation of the different hydrogen-bonding networks in the final aggregate. This hypothesis was supported by the disappearance of the emission peak at $530 \mathrm{~nm}$ in the self-assembled nanostructures of PEG ${ }_{M W 1300}-\mathrm{F} 6$, a monomer with the same PEG moiety of PEG24-F6, but lacking amide groups. 
Two-column images show passive optical waveguiding for three laser beams with wavelengths (from the top to the bottom) of 635, 520, and $450 \mathrm{~nm}$ in the PEG12-F6 peptide film. Optical waveguides and diffractive gratings for light beam coupling and decoupling were fabricated by Focus Ion Beam [133].

The intriguing optoelectronic properties of PEGylated-F6 peptides indicate their promising wide applications in precision nanomedicine and integrated biooptics. In this context, recently, Apter et al. tried to clarify the photonic effects in new bioinspired PEGylated hexaphenylalanine amyloid-like fibers and films [133]. Homogeneous peptide films with a very large area $\left(40 \mathrm{~mm}^{2}\right)$, a low surface rugosity, and a thickness of 6-12 $\mu \mathrm{m}$ were simply prepared by drop-casting the peptide solutions on a glass substrate (Figure 12). The resulting film was characterized by low optical absorbance and a relatively high refractive index. Photonic circuits were simply fabricated on peptide films by patterning the film by the surface focused ion beam (FIB) milling technique. Passive light propagation for red $(635 \mathrm{~nm})$, green $(520 \mathrm{~nm})$, and blue $(450 \mathrm{~nm})$ monochromatic laser light beams was efficiently detected with a good lateral light confinement in the rib waveguides. Peptide films showed a classic optical confinement of the FL and a passive waveguiding propagation. On the other hand, PEG-F6 amyloidogenic fibers exhibited a unique continuous spectrum occupying the entire visible region. Indeed, the full overlap of the FL spectrum and continuous optical absorbance generates a propagation of fluorescence over a long distance (hundreds of micrometers). This evidence was explained by introducing a novel model for the photon reabsorption for the anomalous long-range propagation of FL in PEG-F6 fibers, which is considered in the next chapter $[133,134]$.

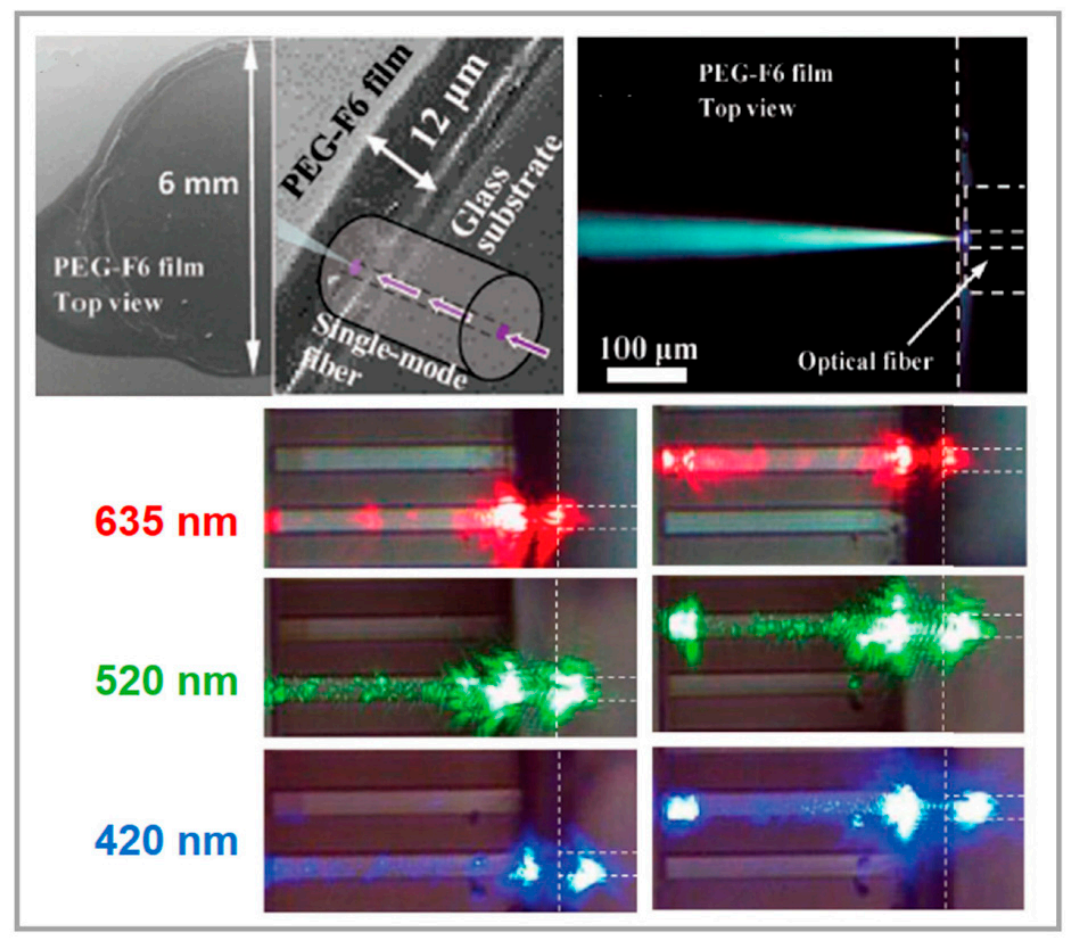

Figure 12. SEM images and optical waveguiding properties of PEG12-F6 peptide film deposited on a glass substrate. Inset images (upper row) show top-view of the PEG12-F6 peptide film. A cleaved edge of the sample allowed to connect a single-mode optical fiber to the edge and to launch the excitation light beam ( $405 \mathrm{~nm}$ laser) into the film which exhibits the waveguiding propagation effect of the excited broadband FL inside the PEG12-F6 peptide film. The dashed lines mark positions of the film edge and optical fiber. (Taken from [133]).

\subsection{Physical Mechanism of Fl in Amyloid and Amyloidogenic Fibrils}

The FL of the PEG-F6 amyloidogenic fibers exhibits a unique continuous spectrum occupying the entire visible region, owing to the additional strong green FL [95,133,134]. This FL spectrum 
completely covers the OA spectrum of these amyloid-like fibers. Despite the full overlap of FL and OA spectra with a high OA coefficient, the FL light propagates in the PEG-F6 fibers for an unexpectedly long range of hundreds of micrometers, exceeding the length determined by classical light absorption by three to six times the corresponding wavelength throughout the visible region (Figure 13) [133,134]. Such anomalous FL transmission indicates an exceptional physical mechanism guiding the long-range FL propagation. It should be stressed that the excited FL of the amyloid-like $\beta$-sheet fibers demonstrates very low optical confinement, showing strong FL scattering during its propagation along the fiber (Figure 13). This suggests that the submillimeter FL long-range propagation in these bioinspired amyloidogenic fibrillar structures is governed by the physical mechanism that is based on photon reabsorption.

(a)
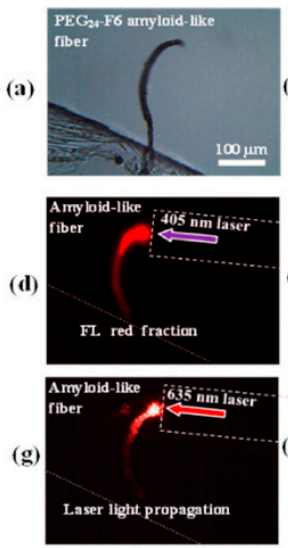
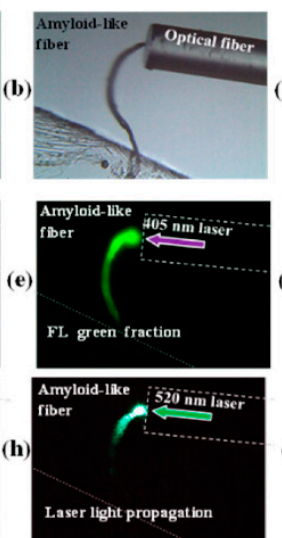
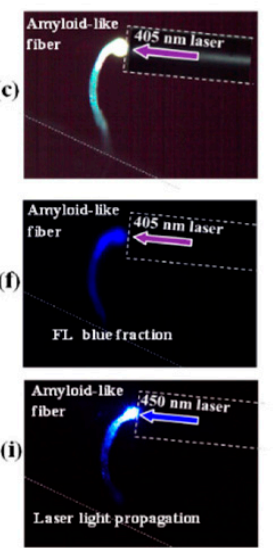
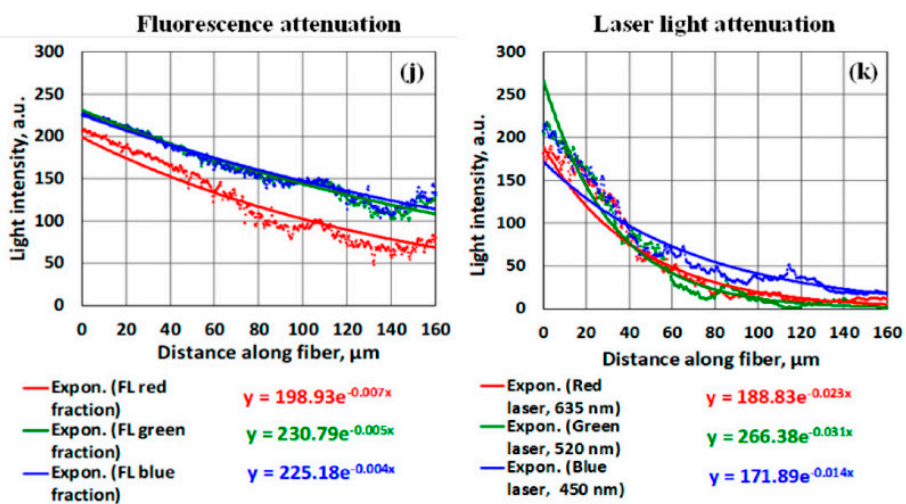

Figure 13. Fluorescence and laser light propagation in $\mathrm{PEG}_{24}$-F6 fiber (a) Free-standing $350 \mu \mathrm{m}$-long $\mathrm{PEG}_{24}-\mathrm{F} 6$ amyloid-like fiber. (b) Single-mode optical fiber connected to $\mathrm{PEG}_{24}-\mathrm{F} 6$ amyloid-like fiber. (c) FL propagation in $\mathrm{PEG}_{24}$-F6 amyloid-like fiber excited by violet laser $(405 \mathrm{~nm})$. The images (d), (e), and (f) show the red (600-650 nm), green $(510-560 \mathrm{~nm})$, and blue $(430-480 \mathrm{~nm})$ fractions of broadband FL. The images (g), (h), and (i) demonstrate the propagation of non-fluorescent, narrow-band optical radiation from three different laser sources, operating at wavelengths $635 \mathrm{~nm}$ (red), $520 \mathrm{~nm}$ (green), and $450 \mathrm{~nm}$ (blue), respectively. (j) Attenuation characteristics of red $(600-650 \mathrm{~nm})$, green $(510-560 \mathrm{~nm})$, and blue $\left(430-480 \mathrm{~nm}\right.$ ) fractions of the broadband FL radiation, propagating in $\mathrm{PEG}_{24}-\mathrm{F} 6$ amyloidogenic fiber. (k) Attenuation characteristics of narrow-band, red $(635 \mathrm{~nm})$, green $(520 \mathrm{~nm})$, and blue $(450 \mathrm{~nm})$ laser radiation, propagating in the same PEG24-F6 amyloidogenic fiber. The dots on the left and right panels correspond to the experimental data, and the solid curves represent exponential fits. (Taken from [133]).

A new approach to FL long-range propagation in amyloid-like fibers was developed in [133]. Bearing in mind the model of photon reabsorption used before in the description of light energy transfer 
in gases and molecular crystals for forming excitons [135], the equation for the excited electronic state population $n_{2}$ can be written as [136-138]:

$$
\frac{\partial n_{2}(z, t)}{\partial t}=\int \sigma_{a}(\omega) \widetilde{J}(z, t ; \mathbf{n}, \omega) v(\omega) \eta(\omega) d \omega d \Omega-\frac{n_{2}(z, t)}{T_{1}}
$$

where $T_{1}$ is the lifetime of the excited state; $\sigma_{a}(\omega)$ is the cross-section of the photon absorption at frequency, $\omega ; v(\omega)$ is the photon group velocity; $\eta(\omega)$ is that part of the absorption processes of the photon of frequency $\omega$ that leads to excited molecular generation; $d \Omega$ refers to the integration in the solid angle $(d \Omega=\sin \theta d \theta d \phi)$; and the concentration of photons is $\widetilde{J}(z, t ; \mathbf{n}, \omega)$, with $\mathbf{n}$ being the unit vector that determines the direction of its velocity.

The equation for the photon concentration $\widetilde{J}(z, t ; \mathbf{n}, \omega)$ is as follows from [135]

$$
n_{z} \frac{\partial \widetilde{J}(z, t ; \mathbf{n}, \omega)}{\partial z}=N \frac{F_{f}(\omega)}{4 \pi T_{1} v(\omega)} n_{2}(z, t)-N \sigma_{a}(\omega) \widetilde{J}(z, t ; \mathbf{n}, \omega)
$$

where function $F_{f}(\omega)$ determines the shape of the FL spectrum $\left(\int F_{f}(\omega) d \omega=1\right)$ and $N$ is the density of molecules. The second term on the right-hand side of this Equation describes the FL decay due to classical absorption, and the first term is the amplification of FL due to the excited molecules created by the light absorption (reabsorption).

For steady-state conditions, we have Equation (1)

$$
n_{2}(z)=T_{1} \int \sigma_{a}(\omega) \widetilde{J}(z ; \mathbf{n}, \omega) v(\omega) \eta(\omega) d \omega d \Omega
$$

Substituting the last equation into Equation (2), we get

$$
n_{z} \frac{\partial \widetilde{J}(z ; \mathbf{n}, \omega)}{\partial z}=-N \sigma_{a}(\omega) \widetilde{J}(z ; \mathbf{n}, \omega)+\frac{F_{f}(\omega)}{4 \pi v(\omega)} N \int \sigma_{a}(\omega) \widetilde{J}(z ; \mathbf{n}, \omega) v(\omega) \eta(\omega) d \omega d \Omega
$$

The last equation shows that $\widetilde{J}(z ; \mathbf{n}, \omega)$ decays slower than $\sim \exp \left[-N \sigma_{a}(\omega) z\right]$.

To proceed, one needs to perform an expansion in terms of the eigenmodes of a waveguide. To make evaluations of a large diameter of the waveguide $(\sim 16 \mu \mathrm{m})$, we suppose an isotropic distribution of $\widetilde{J}(z ; \mathbf{n}, \omega)=\widetilde{J}(z, \omega)$ and a weak dependence of $v(\omega)$ and $\eta(\omega)$ on frequency $\omega$. Then, integrating both sides of Equation (4) with respect to $\omega$ within a definite FL band, we get

$$
\frac{\partial J(z)}{\partial z}=-(1-\eta) N \int \sigma_{a}(\omega) \widetilde{J}(z, \omega) d \omega
$$

where $\eta \leq 1$ and $J(z)=\int \widetilde{J}(z, \omega) d \omega$, and we consider that $\int d \Omega=4 \pi$.

If the dependence of $\sigma_{a}(\omega)$ on frequency is weak within a definite FL band, one can write $\int \sigma_{a}(\omega) \widetilde{J}(z, \omega) d \omega \approx \bar{\sigma}_{a} \int \widetilde{J}(z, \omega) d \omega=\bar{\sigma}_{a} J(z)$, where $\bar{\sigma}_{a}$ is the average value of $\sigma_{a}(\omega)$ in the FL band. Then, Equation (5) gives

$$
\frac{\partial J(z)}{\partial z}=-(1-\eta) N \bar{\sigma}_{a} J(z)
$$

The solution of the last equation is

$$
J(z) \sim \exp \left[-(1-\eta) N \bar{\sigma}_{a} z\right]
$$

where $\eta \leq 1$ is the fitting parameter. Our experimental results, presented in Figure 13j,k demonstrate $\mathrm{FL}$ and laser light propagations in free-standing $\mathrm{PEG}_{24}-\mathrm{F} 6$ amyloid-like fibers. These results are accumulated in Table 2 and facilitate the estimation of the parameter $\eta$. 
Table 2. Basic parameters of FL and monochromatic light propagation in $\mathrm{PEG}_{24}-\mathrm{F} 6$ fibers.

\begin{tabular}{cccccc}
\hline \multirow{2}{*}{$\begin{array}{c}\text { Spectral } \\
\text { Range }\end{array}$} & $\begin{array}{c}\text { Attenuation Coefficient } \\
\begin{array}{c}\text { Narrow-Band } \\
\text { Laser } \\
\text { Radiation } \\
\alpha_{L},\left(\mathbf{c m}^{-1}\right)\end{array}\end{array}$ & $\begin{array}{c}\text { Characteristic Propagation Length } \\
\text { Badiation } \\
\boldsymbol{\alpha}_{F L},\left(\mathbf{c m}^{-1}\right)\end{array}$ & $\begin{array}{c}\text { Narrow-Band } \\
\text { Laser Radiation } \\
L_{L}=1 / \boldsymbol{\alpha}_{L} \boldsymbol{\prime}(\mu \mathrm{m})\end{array}$ & $\begin{array}{c}\text { Broadband FL } \\
\text { Radiation } \\
\boldsymbol{L}_{F L}=1 / \alpha_{F L},(\mu \mathrm{m})\end{array}$ & $\boldsymbol{L}_{F L} / L_{L}$ \\
\hline Red & 230 & 70 & 44 & 143 & 3.3 \\
\hline Green & 310 & 50 & 32 & 200 & 6.2 \\
\hline Blue & 140 & 40 & 71 & 250 & 3.5 \\
\hline
\end{tabular}

From the comparison between Equation (7) and exponential decay fits (Figure 13j,k, Table 2) we get $\eta$ values of approximately $0.7,084$, and 0.7 for the red $(600-650 \mathrm{~nm})$, green $(510-560 \mathrm{~nm})$, and blue (430-480 nm) fractions of FL, respectively. Equation (7) and the estimation are fully consistent with our experimental results on FL attenuation in amyloidogenic $\mathrm{PEG}_{24}-\mathrm{F} 6$ fibers (Figure 13j,k), where $J(z) \sim \exp \left(-N \sigma_{a} z\right)$ at $\eta=0$ and $N \sigma_{a}=\alpha_{L}$ describes classical absorption.

These amyloidogenic structures exhibit exclusive broadband, fully overlapped FL and OA spectra, which are created by the electronic structure of noncovalent hydrogen bonds also responsible for visible FL in amyloid structures [85-87]. These found FL and OA effects are detected along the entire visible spectrum in the 400-650 $\mathrm{nm}$ range [133]. This effect provides the observation of any FL visible color: blue, green, yellow, red, and more. The reabsorption effect depends on the product of the equilibrium absorption and luminescence spectra (see Equation (4)); in these wide spectral regions, any optically absorbed photon can be reemitted, yielding an FL photon, and any emitted FL photon can be absorbed. Thus, there is no Stokes shift in the considered reabsorption process. The excitation of the FL by a laser source is followed by its propagation inside the $\mathrm{PEG}_{24}-\mathrm{F} 6$ fiber, owing to consecutive acts of light absorption, followed by FL photon propagation and reabsorption Figure 14, provided by the overlapped transitions in our system.

This process is based on the multi-well potential structure created by hydrogen bonds $[87,88]$, which is schematically described for only one band by the simplified system (Figure 14), showing excitation, relaxation, FL irradiation, and FL photon reabsorption. This complex FL propagation process occurs along all FL centers in the amyloid-like fibers, generating a wide FL visible continuous spectrum.

The proof-of-concept of a long-range FL probe was realized by testing conical $\mathrm{PEG}_{24}$-F6 fibers of $1.5 \mathrm{~mm}$ in length [130]. FL was excited by the $405 \mathrm{~nm}$ laser beam normally incident to the fiber axis, which exhibits quasi-white FL along the laser beam propagation (Figure 14). This disc-shaped FL region is widened and transmitted laterally in up and down directions, demonstrating a gradual FL decrease due to the FL attenuation. It propagates at a distance close to $800 \mu \mathrm{m}$, which is consistent with the developed model of FL reabsorption. The FL signal outcouples the fiber and illuminates the dark screen. The FL of the fiber gradually changes in its intensity and spectrum. Its quasi-white color is modified, and at the fiber end, it exhibits a green-like tint (bottom row, Figure 14d). Such a coloration is the evidence of the smallest attenuation coefficient of the green FL component. This was directly demonstrated in our experiments, which include spectral studies of different FL fractions in $\mathrm{PEG}_{24}-\mathrm{F} 6$ amyloid-like fibers [133] and the demonstration of green FL long-range propagation in the fiber, supported by direct measurements of its attenuation coefficient (Figure 13). The most distinguishing feature of the described FL phenomenon is a wide FL spectrum covering the entire visible region. Figure 14 shows bright blue $(430-480 \mathrm{~nm})$, green $(510-560 \mathrm{~nm})$, and red $(600-650 \mathrm{~nm}) \mathrm{FL}$ fractions generated by the fiber and reaching the screen. Such a rich continuous FL spectrum, shown in the presented proof-of-concept, opens an avenue for the development of tunable light nanoprobes from new biocompatible materials and their application in photomedicine (optical biopsy, optogenetics, and other light diagnostics and therapy methods). 

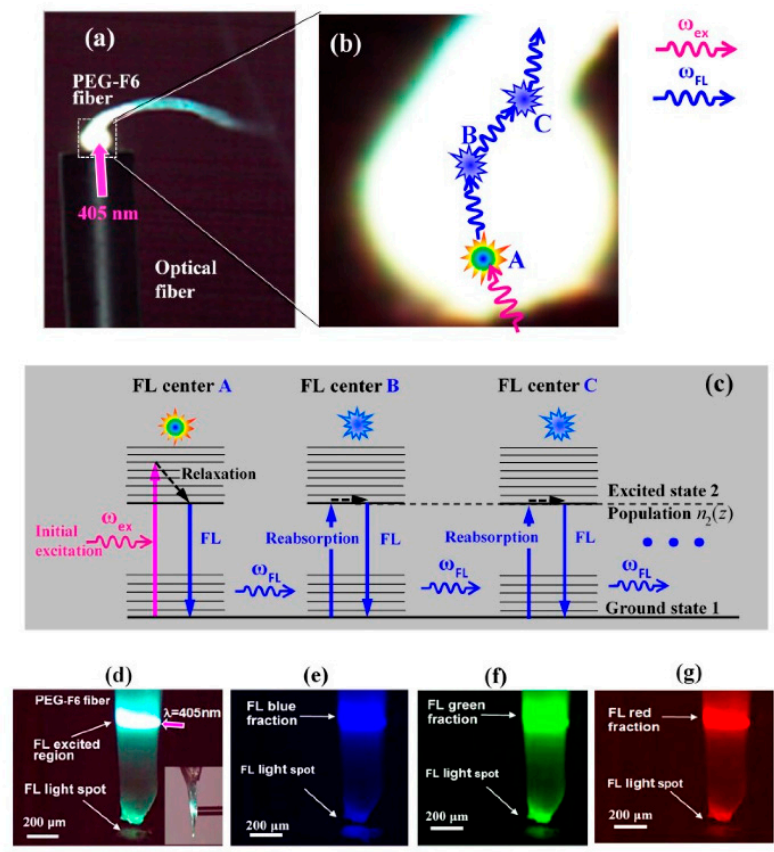

(g)

Figure 14. Scheme of consecutive elementary acts of excitation, FL emission, and reabsorption, involved in long-range light propagation in amyloid-like fibers. (a) Quasi-white FL, excited in $\mathrm{PEG}_{24}$-F6 fiber. (b) Schematic presentation of exciting photon absorption, FL generation, and reabsorption along the $\mathrm{PEG}_{24}$-F6 fiber. The A-B-C path presents one of many possible directions of light energy flow from initially excited FL center A to the next FL centers B, C involved in FL light propagation along the fiber at any FL frequency $\omega_{\mathrm{FL}}$. (c) Simplified energy diagrams of excitation, FL, and reabsorption transitions contributing to the FL propagation along the PEG-F6 fiber. The FL centers A, B, C correspond to those, shown in the scheme (b). Bottom row, Proof-of-Concept: FL propagation in grown amyloid-like fiber for $\sim 800 \mu \mathrm{m}$; (d) FL (full spectrum) propagation excited by $405 \mathrm{~nm}$ laser; (e-g) FL blue, green, and red spectral fractions illuminating the screen. (Taken from [133]).

\section{Visible Fluorescence Bionanodots}

Subjected to biological refolding the bioinspired peptide/protein structures adopt -sheet fold and reveal multicolor intensive visible FL [2,3,89-93]. This intrinsic biophotonic effect found in $\beta$-sheet 1D-fibrillary structures and 2D-hybrid polymer/peptide thin films of different composition, morphology and dimensions exhibit unique similar FL in a wide visible range of 400-700 nm. In this chapter, we present our recent development of new visible FL 0D-biodots and their applications $[3,93]$. These bio-organic nanoparticles, composed from a variety of synthetic short FF, LL, FFF peptides, and insulin amyloid protein do not possess any visible FL in their native state. However, they adopt FL properties under the thermally mediated refolding of their biological secondary structure from their original metastable helix-like to a stable $\beta$-sheet structure. These inherently biocompatible visible FL peptide/protein nanodots (PNDs) can be considered promising nanoscale bioimaging markers with super-high resolution for emerging precise medicine.

\subsection{Fabrication Technology of Visible Bionanodots}

The fabrication process of visible nanodots consistsed of two steps. In the first step, PNDs were self-assembled from monomer peptide molecules in organic solvents by a bottom-up method [121,139], where the peptide/protein monomer nucleates to seeds with a critical size, which can be considered as building blocks in terms of the supramolecular concept. The details of the FF, LL, FFF and insulin nanodot fabrication can be found in the Materials and Methods section. The common feature of this nanotechnology is based on the inhibition of the self-assembly process of native peptide/protein biomolecules at the first initial stage of nucleation to nanodots by the use of polar solvents. In the second 
step, the PNDs were subjected to a thermal treatment at $160-180{ }^{\circ} \mathrm{C}$ in a high boiling temperature solvent (ethylene glycol; boiling point: $195^{\circ} \mathrm{C}$ ) and then cooled down back to room temperature. This procedure allowed us to convert the nonfluorescent PNDs to visible FL-PNDs with a refolded secondary structure (Figure 15).

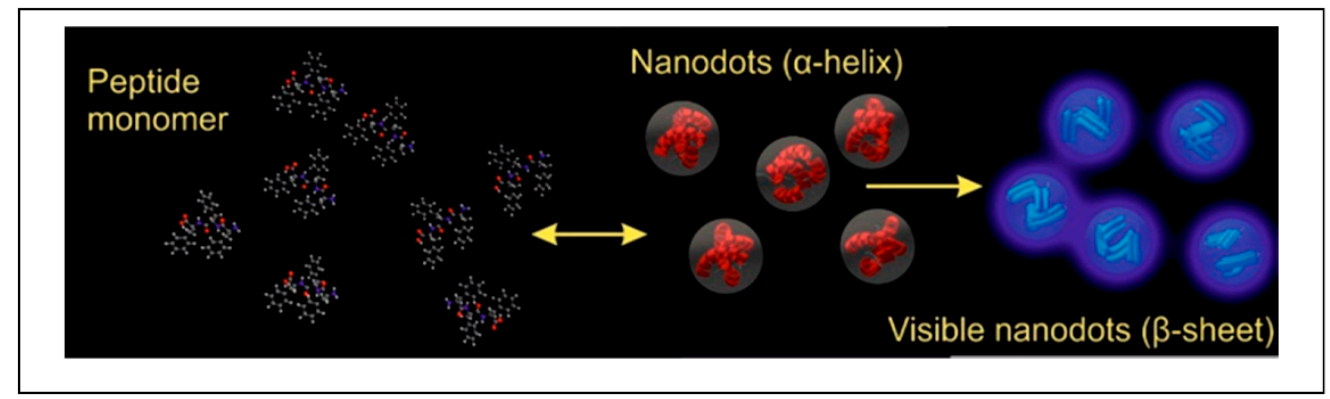

Figure 15. Formation process of FL visible peptide/protein nanodots (PNDs). At the first stage, due to molecular recognition, individual peptide/protein molecules are self-organized into nanodots having a helix-like peptide secondary structure. At the next stage, these nanodots are subjected to the thermal treatment up to $160{ }^{\circ} \mathrm{C}$ for $3 \mathrm{hs}$ in order to refold the original biomolecular organization from helix-like to $\beta$-sheet secondary structure. Such refolding leads to the onset of the unique tunable visible fluorescent properties of PNDs. (Taken from [3]).

High-resolution microscopy techniques, namely environmental scanning electron microscopy (ESEM), atomic force microscopy (AFM) and transmission electron microscopy (TEM) were exploited for morphological analyses of the PNDs [3,93]. The images of the FFF-PNDs are demonstrated in Figure 16, which shows individual FFF-PNDs with an average height and lateral dimensions of $\sim 10 \mathrm{~nm}$ (Figure 16a-d). Inspection of our other FF-, LL- and insulin PNDs showed lower dimensions with the average diameters as follows: FF dots $\sim 3 \mathrm{~nm}$, LL dots $\sim 3.2 \mathrm{~nm}$, insulin dots $\sim 5 \mathrm{~nm}[3,139]$.

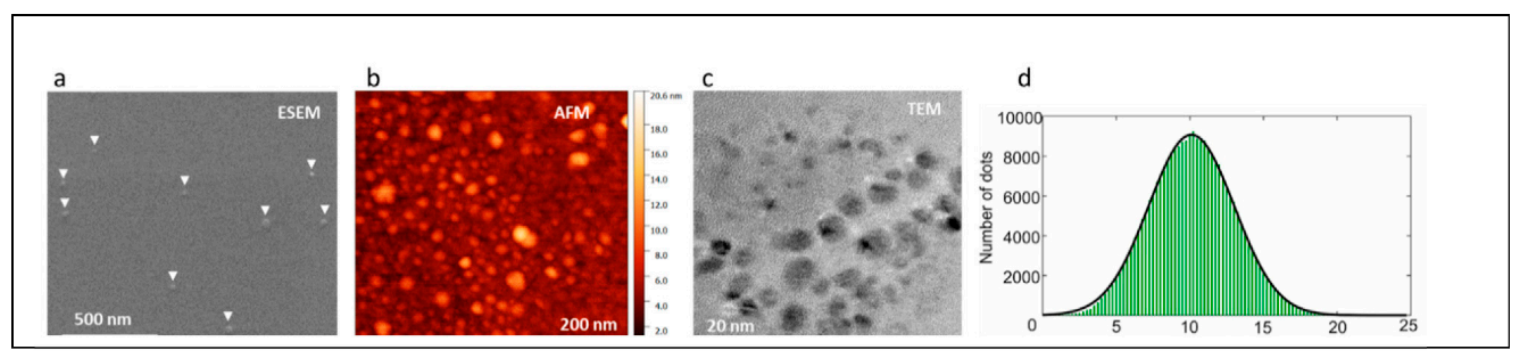

Figure 16. Morphological characterization of FFF nanodots. (a-c) environmental scanning electron microscopy (ESEM), AFM, and TEM images of self-assembled FFF nanodots, respectively. (d) Height distribution of FFF nanodots obtained from AFM measurement representing the average Z-dimension of nanodots $\sim 10 \mathrm{~nm}$. According to TEM characterization, the average $X-Y$ dimension is $\sim 10 \mathrm{~nm}$. (Taken from [3]).

\subsection{Optical Properties of Pnds Under the Refolding of Peptide Secondary Structure}

The optical absorption, OA, FL spectroscopy, and FL lifetime were studied for native and thermally treated FFF-PNDs, where a profound difference in the optical properties for native and thermal treated nanodots was observed (Figure 17). The OA for native FFF-PNDs (Figure 17a) exhibits its absorption edge in the region of $\lambda \sim 250-270 \mathrm{~nm}$ which is similar to that observed in other different peptide nanostructures, FF nanotubes, FFF nanospheres and FFF nanoplates [2,89,90,107]. This OA is associated with the aromatic phenyl rings of the F-phenylalanine residues [120]. As expected, aliphatic L-leucine amino acid and native LL peptide nanodots, nanotubes, and nanofibers do not exhibit any OA in this 
UV spectral interval [2,120]. Thus, both F-aromatic and L-aliphatic-based PND in their native states are entirely transparent in a wide optical region covering visible and near IR regions.
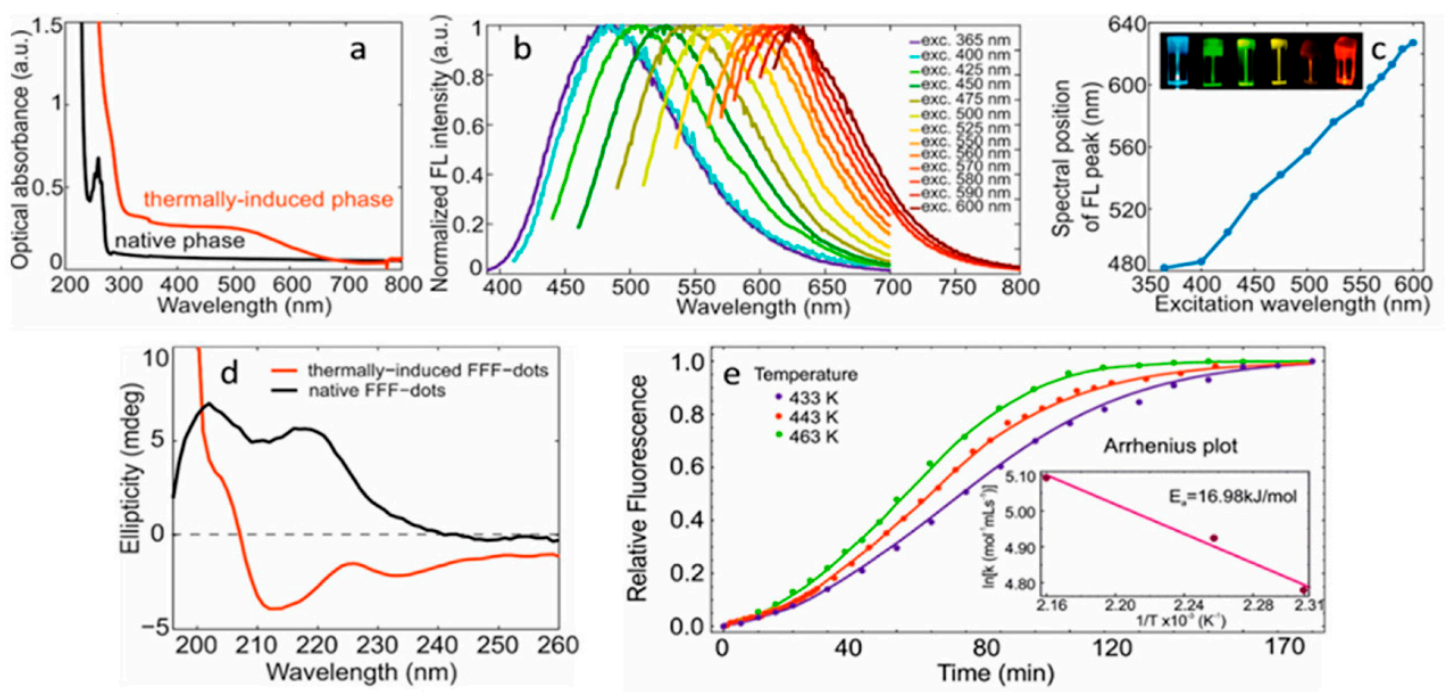

Figure 17. Optical properties of native and thermally treated FFF nanodots. (a) Optical absorption (OA) of native (black line) and thermally treated (red line) nanodots. (b) Normalized FL spectra of thermally treated nanodots vs. excitation wavelength, $\lambda$ exc, varied in the range of 365-600 nm. (c) The FL photon emission peak vs. $\lambda$ exc. Inset shows color images of thermally treated FFF nanodots at different excitation wavelengths (from left to right: $\lambda \operatorname{exc}=360 \mathrm{~nm}, \lambda \operatorname{exc}=450 \mathrm{~nm}, \lambda \operatorname{exc}=510 \mathrm{~nm}, \lambda$ exc $=530 \mathrm{~nm}$, $\lambda$ exc $=570 \mathrm{~nm}, \lambda$ exc $=590 \mathrm{~nm}$ ) demonstrating continuous variation in the FL spectrum. (d) Circular dichroism (CD) spectra of FFF nanodots in native (black line) and thermally induced (red line) phases illustrating the conformational transition from helix-like to $\beta$-sheet secondary structure. (e) Monitoring of the refolding kinetics in FFF nanodots by FL measurements at three different temperatures of the heat treatment ( $433 \mathrm{~K}, 443 \mathrm{~K}$ and $463 \mathrm{~K}$ ). The excitation and emission FL wavelengths were $404 \mathrm{~nm}$ and $530 \mathrm{~nm}$, respectively. Fitting of the experimental data was performed by the Arrhenius equation (Equation (8)). The inset shows the reaction rate (in the log scale) vs. the inverse temperature. It allows the linear fitting that gives an activation energy of $16.98 \mathrm{~kJ} / \mathrm{mol}$ and frequency factor $\mathrm{A}=13500 \mathrm{~mol}^{-1} \mathrm{mLs}^{-1}$. (Taken from [3]).

Thermal treatment of the studied self-assembled di-FF and tri-FFF-PNDs led to a significant transformation of their optical properties (Figures 17 and 18). The OA spectrum of the heated FFF nanodots (red graph) demonstrates a new broadband OA and FL properties covering the entire visible region, 400-650 $\mathrm{nm}$. The most distinguishing feature of this acquired visible FL effect is its excitation dependence, making FL tunable in a very wide visible range from deep blue $(440 \mathrm{~nm})$ to red $(650 \mathrm{~nm})$ (Figure 17b,c). It is also illustrated by a linear graph of FL peaks vs. excitation wavelengths (Figure 17c and inset in c) of several color FL images of heated FFF nanodots excited by different excitation wavelengths. It should be noted that we checked the Stokes signal (peak FL line) as a function of the excitation wavelength. This graph clearly shows that FL in our systems is a linear process. The measured quantum yield (QY) of FFF dots in the blue region ( $\lambda$ exc $\sim 380 \mathrm{~nm}$, $\lambda \mathrm{em} \sim 460 \mathrm{~nm}$ ) showed relatively a high-value QY of $\sim 30 \%[3]$. 

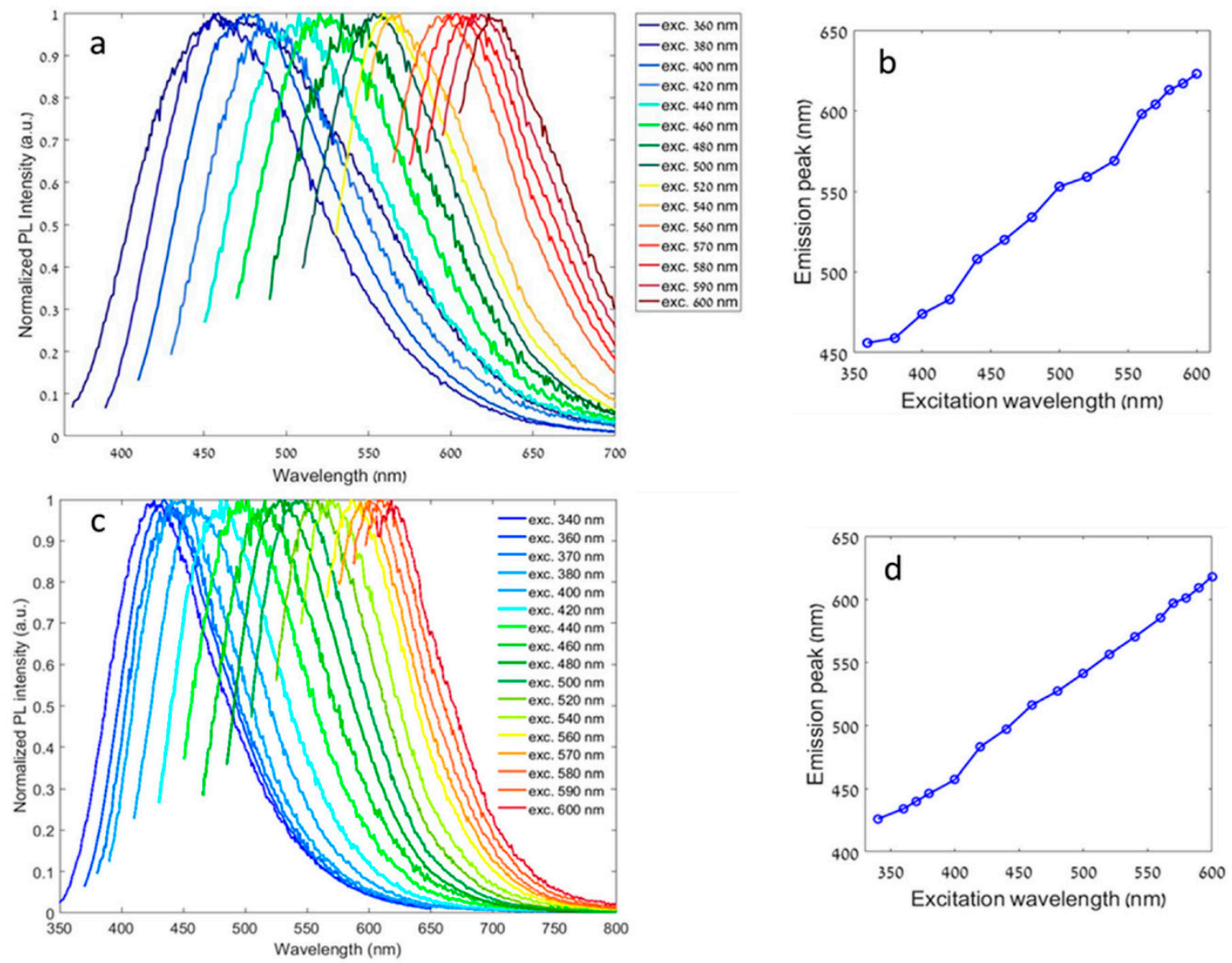

Figure 18. Normalized FL spectra and dependence of FL peak vs. $\lambda$ exc of thermally treated $\beta$-sheet $(\mathbf{a}, \mathbf{b})$ FF nanodots and $(\mathbf{c}, \mathbf{d})$ LL nanodots.

Regardless of the original composition, similar FL properties were also observed both in aromatic FF- and aliphatic LL-PNDs subjected to the heat treatment (Figure 18). It should be noted that the dimensions of FF and LL nanodots are much smaller by a factor of $\sim$ three to four than those of FFF dots, though they demonstrate similar FL spectra. The found similarities between FL properties from di- and tri-PNDs of different compositions, origins, and dimensions at the nanoscale distinctly show that visible FL is not related to the quantum confinement effect found in inorganic nanodots [10]. This remarkable FL induced by the thermal treatment indicates the structural biomolecular $\beta$-sheet reorganization of the PNDs upon heat treatment and is fully consistent with previously demonstrated FL results studied for FFF, FF ad LL microstructures (Figure 10).

The latter assumption is supported by our circular dichroism measurements of the peptide secondary structure [112,117]. The CD spectrum of the native non-treated FFF-PND (Figure 17d, black line) exhibits two positive molar ellipticity maxima at $203 \mathrm{~nm}$ and $219 \mathrm{~nm}$. Such a CD spectrum describes a left-handed helix-like state, where the observed peaks are related to $\pi-\pi^{*}$ and $n-\pi^{*}$ electronic transitions, respectively [140,141].

Following thermally induced refolding of the FFF-PNDs, the CD spectrum changes dramatically, and it becomes negative with ellipticity minimum at $214 \mathrm{~nm}$ (Figure 17d, red line). This negative peak and its spectral position are consistent with classic CD data recorded with an antiparallel $\beta$-sheet secondary structure [88,113]. The CD measurements (Figure 17) clearly show a structural transition of the FFF-PNDs from helical conformation to a new $\beta$-sheet secondary structure. A similar conformational transition from $\alpha$-helix to $\beta$-sheet secondary structure is well known and observed in protein/peptide misfolding and subsequent $\beta$-sheet-rich amyloid fibrillary structure formation $[77,78]$. 
It should be noted that it is commonly thought that small linear peptides between three and six amino acids in length cannot exhibit a helix-like conformation due to size restrictions, but the ability of such ultra-small peptides to form $\alpha$-helical structures upon crossing a threshold concentration was recently shown $[142,143]$.

Despite different compositions and origins, both di-peptides exhibit fold-sensitive FL and OA spectra similar to all those FL data found in bioinspired and biological amyloid and amyloidogenic fibrillary $\beta$-sheet structures.

\subsection{Monitoring of the Secondary Structure and Its Kinetics Under Refolding Helix-Like $\rightarrow \beta$-Sheets}

The found thermally induced transformation $\alpha$-helix $\rightarrow \beta$-sheets can be associated with their own set of kinetic rates. For experimental verification, the FFF nanodots in their initial helix-like phase were heated quickly to elevated temperatures. The conformational rate from initial metastable helix-like to fluorescent $\beta$-sheets was monitored by the relative FL intensity (excitation $\lambda$ exc $=404 \mathrm{~nm}$, emission $\lambda \mathrm{em}=530 \mathrm{~nm}$ ) as a function of time at three different temperatures $433 \mathrm{~K}, 443 \mathrm{~K}, 463 \mathrm{~K}$ (Figure 17e). Such a classical approach allowed us to extract the natural activation energy barrier, associated with the transition to the $\beta$-sheet structure upon heat treatment. The analysis of our observed thermally induced transition can be performed in the framework of the standard autocatalytic model [144], which can be written as:

$$
\begin{aligned}
& \frac{d P_{\alpha}}{d t}=-k P_{\beta} P_{\alpha}, \\
& \frac{d P_{\beta}}{d t}=k P_{\beta} P_{\alpha},
\end{aligned}
$$

where $P_{\alpha}$ and $P_{\beta}$ denote the relative aggregate concentration in helix-like and $\beta$-sheet phases, respectively, and $k$ stands for the reaction rate. The solution of these equations is given by:

$$
\begin{aligned}
& P_{\alpha}(t)=\frac{1}{1+\frac{P_{\beta 0}}{P_{\alpha 0}} \exp (-k t)}, \\
& P_{\beta}(t)=1-P_{\alpha}(t)
\end{aligned}
$$

with $P_{\alpha 0}$ and $P_{\beta 0}$ corresponding to the initial relative aggregate concentration, where $P_{\alpha 0}+P_{\beta 0}=1$. Within this model, we assume that the concentration of the FFF nanodots is invariant while the transitions concern their internal structure only.

Fitting experimental data with Equation (8), shown in Figure 17e, allowed us to retrieve the initial aggregate concentrations of $P_{\alpha 0}=0.91$ and $P_{\beta 0}=0.09$ for all samples under the study. Plotting the extracted logarithm of the reaction rates at different temperatures as a function of inverse temperature (inset in Figure 17e) allowed fitting to the Arrhenius equation:

$$
k=A \exp \left(-\frac{E_{a}}{R T}\right)
$$

where the frequency factor $A$ accounts for the frequency of collisions, $E_{a}$ is the activation energy, and $R$ is the gas constant. The value of the frequency factor $A$ has been obtained from the Arrhenius plot, accounting for the fact that the initial concentration of monomers is $1 \mathrm{mg} / \mathrm{mL}$ and their molar mass is $459.55 \mathrm{~g} / \mathrm{mol}$ [145]. Our fitting results gave estimations of the activation energy of $E_{a}=16.98 \mathrm{~kJ} / \mathrm{mol}$ and the frequency factor of $A=13500 \mathrm{~mol}^{-1} \mathrm{mLs}^{-1}$.

Activation energy is an important thermodynamic parameter, which characterizes the governing thermally activated process. In our case, the governing process is the structural thermally induced biomolecular transformation from helix-like to $\beta$-sheet states and the activation energy is the energy barrier needed for this refolding of amyloid origin. The thermodynamic approach was applied to studies of metastability of native proteins and the phenomenon of amyloid formation for a series of amyloid proteins differed in the number of residues [81]. In the cited work, the considered governing process is the refolding of soluble native $\alpha$-helical amyloid proteins to solid-state insoluble $\beta$-sheet amyloid fibrils. The developed technique [81] was based on measurements between the concentration 
of amyloid protein molecules in solution and those incorporated into insoluble fibrils, which is the process of elongation (growth) of amyloid fibers. Our FL method considers the variation in the FL intensity during the fibrillization process (Figure 17e). The FL intensity is proportional to the grown $\beta$-sheet concentration transformed from $\alpha$-helical biomolecules. Our calculated activation energy $E_{a}=16.98 \mathrm{~kJ} / \mathrm{mol}$ is of the same order of magnitude as the activation energy for the elongation process of the amyloid fibrils, which also involves a transition to the $\beta$-sheet structure [81].

\subsection{Visible FL in Amyloid Insulin Nanodots}

The conducted experimental research allowed us to reveal that visible FL properties can be acquired by bioinspired peptide/protein nanostructures as a result of the fundamental reconstruction of their primary $\alpha$-helical state to a $\beta$-sheet one. The studied OA and FL spectra of any $\beta$-sheet peptide/protein structure are completely similar to those observed in amyloid diseased-related fibrils, regardless of their chemical composition, morphology, and dimensions and can be related to fold-sensitive phenomena. These results have encouraged us to explore similar optical phenomena in synthetic amyloid insulin protein nanodots as those observed in bioinspired FFF, FF, and LL nanodots. Insulin protein, as a common model of amyloidogenic proteins, was subjected to the same protocol of inhibition of the amyloidogenesis process at the stage of nanodots (Materials and Method section) followed by thermal treatment to induce visible FL by refolding to a $\beta$-sheet structure. Figure 19 exhibits OA and FL of insulin nanodots, subjected to thermally induced refolding. In the heated insulin dots, the appearance of optical absorbance and excitation-dependent visible FL emission was found. These spectra have optical characteristics similar to the ones of the thermally refolded FFF, FF, LL nanodots. Those revealed in this amyloid protein transformation of optical properties from non-FL to the visible FL-state were observed under similar thermally induced refolding of the insulin dots from a native $\alpha$-helical structure to a $\beta$-sheet-rich structure (Figure 19c).
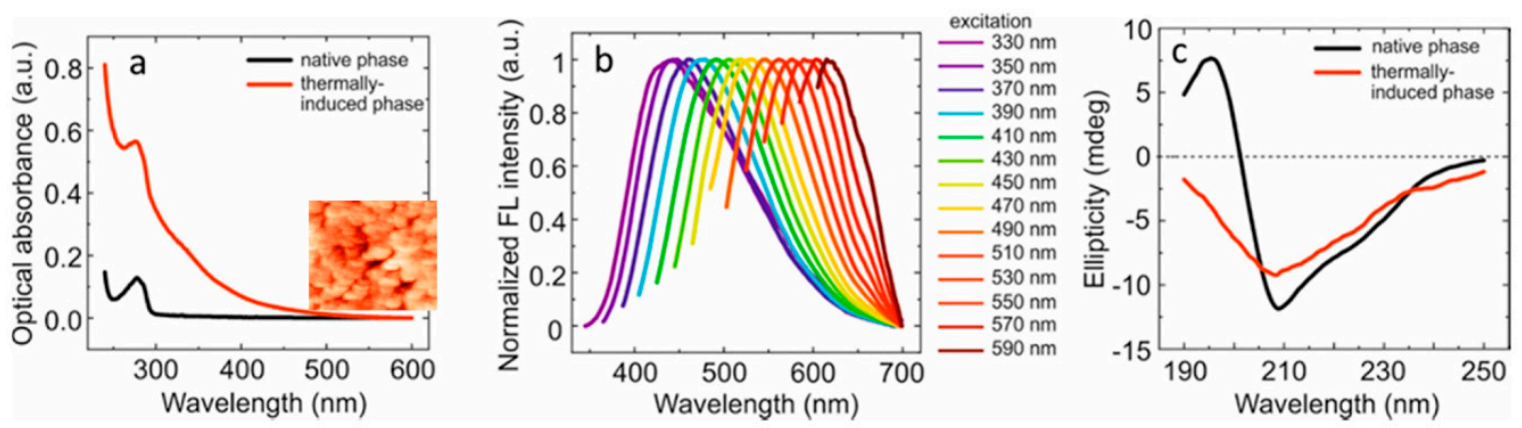

Figure 19. Optical properties of insulin nanodots (inset: AFM image of insulin nanodots) (a) Optical absorption of insulin dots before (black curve) and induced by the thermal treatment (red curve). (b) FL spectra of thermally induced insulin dots vs. the excitation wavelength. (c) Circular dichroism of insulin dots before (black curve) and after thermally induced $\beta$-sheet conversion (red curve). (Taken from [3]).

Studied FFF, FF, LL and insulin peptide/protein $\beta$-sheet nanodots of different origins and compositions exhibit similar spectra of OA and FL as amyloidogenic [2,3,89,90,92,93,104] and amyloid [1,85-88,98] $\beta$-sheet micro-nano-fibrillary structures. These results support the proposed common FL mechanism [87] regarding the universal reconstruction of the original (helix-like state) electronic structure of non-covalent hydrogen bonds when these bonds adopt, in the $\beta$-sheet state, another configuration providing a visible FL effect.

\subsection{Visible Single FL Peptide Nanodots}

To characterize the optical properties of discrete PNDs self-assembled from aromatic FFF peptide biomolecules, we applied single-molecule microscopy methods. Our extensive studies included single 
PND FL imaging, the spectral characterization of the emission, FL lifetime, and emission intermittency measurements [93]. In contrast with previously presented research where all measurements were done in liquid samples (solutions of PNDs in EG), here, to study individual nanoparticles, dry samples were prepared. For the preparation of dry samples with individual nanodots, a solution of FFF-PNDs in spectroscopic grade EG (initial concentration is $1 \mathrm{mg} / \mathrm{mL}$ ) was diluted to 1:1000 in acetone. A drop of this solution was put on a pre-cleaned quartz microscope slide and immediately covered by a pre-cleaned coverslip to immobilize the PNDs and to prevent dust and residue contamination.

We imaged the individual FFF-PNDs with a single-molecule fluorescence microscope (ONI Nanoimager S) equipped with four continuous wave (CW) lasers operating at $375 \mathrm{~nm}, 473 \mathrm{~nm}, 532 \mathrm{~nm}$, and $640 \mathrm{~nm}$. Figure 20 represents an absorption curve of the FFF-PND ensemble in EG solution with corresponding excitation wavelengths marked by arrows. Figure 20b shows epi-fluorescence images of individual PNDs dispersed on the coverslip and excited by four different lasers. It can be seen that the same nine single nanoparticles are fluorescent under $473 \mathrm{~nm}$ and $532 \mathrm{~nm}$ excitations. Particle numbers $1,3,5$, and 9 also emit light under $375 \mathrm{~nm}$ excitation and there is no light emission for the 640-nm excitation, as expected from the optical absorption graph (Figure 20a).

(a)

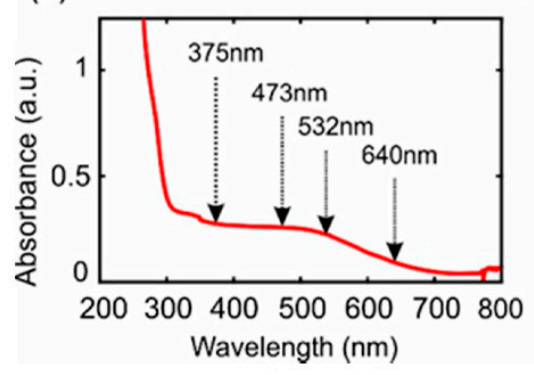

(b) exc. @ 375 nm

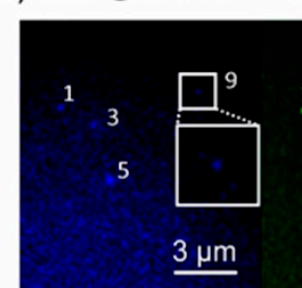

exc. @ 473 nm

exc. @ 532 nm

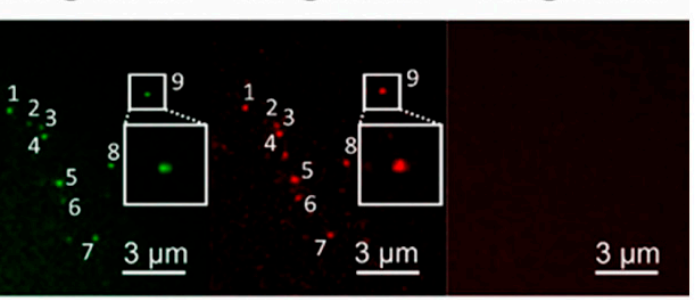

Figure 20. Optical absorption and FL images of single FFF nanodots. (a) Optical absorption curve of FFF-PNDs in ethylene glycol (EG) solution with applied excitation wavelengths marked by arrows. (b) Fluorescence images of the same single peptide dots excited at $375 \mathrm{~nm}, 473 \mathrm{~nm}, 532 \mathrm{~nm}$, and $640 \mathrm{~nm}$ (inserts show the same individual dot \# 9 excited at different wavelengths). Each visible nanodot is indicated by a number. The last image shows that no fluorescence is measured under $640 \mathrm{~nm}$ excitation, which is consistent with the absorbance curve. (Taken from [93]).

In this work [93], we showed that the FL spectra of the single PNDs and their tunability coincide with the spectra and tunability measured for PND ensembles in EG solution. This provides strong evidence that the tunability of PND fluorescence is a property of the individual PNDs and is not a result of an inhomogeneous mixture of different emitters. However, a closer look demonstrated that the single-nanoparticle FL spectra are narrower than the ensemble spectra and that the emission maxima of different PNDs varies over $\sim 30 \mathrm{~nm}$. FL lifetime studies showed short time scale decay with a typical value of several nanoseconds. Along with their relatively high quantum yield, this may indicate their suitability as new biocompatible fluorescent probes as well as optical gain material. Long timescale measurements revealed pronounced fluorescence blinking involving continuous bright states with a duration in the range of seconds. This gives the first evidence of the quantum emitter nature of FL PNDs. It marks PNDs as attractive for various blinking-based super-high-resolution techniques, such as stochastic optical reconstruction microscopy (STORM) [146].

\subsection{Bioimaging as a Prospective Application of the Visible FL Peptide Nanodots}

Thermally treated $\beta$-sheet FL visible PNDs have several attractive properties, such as small nanometer size, multicolor FL emission profiles, tunability, and potential biocompatibility, which make them compelling candidates for FL bioimaging in the visible region. Moreover, the peptide/protein nanodots exhibit excellent photostability, as shown in our photobleaching measurements [93]. 
As proof-of-concept for bioimaging applications of the FL FFF nanodots, we embedded them into porous vaterite semi-spherical particles of micron-scale size (Figure 21)
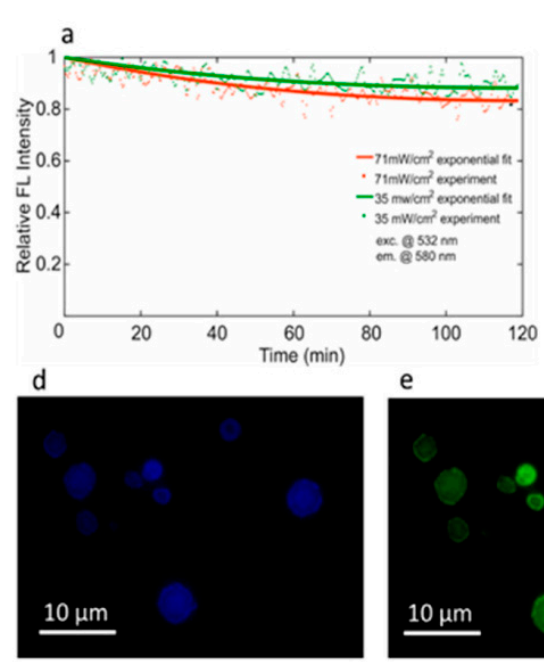

Exc. $365 \mathrm{~nm}$ b

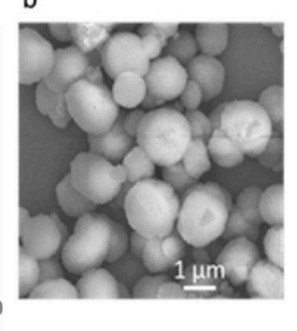

c

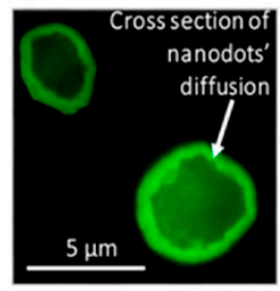

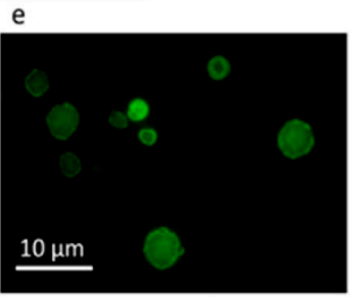

Exc. $425 \mathrm{~nm}$

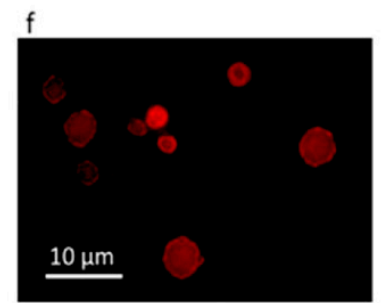

Exc. $570 \mathrm{~nm}$

Figure 21. Bioimaging properties of visible FL FFF nanodots embedded in vaterite micro-particles. (a) Photobleaching of FL FFF nanodots embedded into vaterite microcapsules, FL intensity $\lambda_{\mathrm{FL}}=580 \mathrm{~nm}$ vs. time, $\lambda$ exc $=532 \mathrm{~nm}$, continuous wave $(\mathrm{CW})$ laser at power densities of $71 \mathrm{~mW} / \mathrm{cm}^{2}$ (red curve) and $35 \mathrm{~mW} / \mathrm{cm}^{2}$ (green curve); (b) ESEM image of porous vaterite microspheres. (c) FL microscopy image of two vaterite particles with FFF nanodots embedded in their pores; $(\mathbf{d}-\mathbf{f})$ FL images of vaterite spheres in blue, green, and red spectral regions due to intrinsic FL of embedded FFF nanodots $(\lambda$ exc $=$ $365 \mathrm{~nm}, \lambda \mathrm{exc}=425 \mathrm{~nm}$ and $\lambda \mathrm{exc}=570 \mathrm{~nm}$ ). (Taken from [93]).

Vaterite is one of the polymorphs of calcium carbonate $\left(\mathrm{CaCO}_{3}\right)$. High porosity, extraordinary loading capacity, triggered release mechanisms along with natural biocompatibility and biodegradability make the vaterite particles a very promising platform in biomedicine as a cargo for drug delivery and tissue engineering applications $[147,148]$. The recently developed nanotechnology using porous micro- and vaterite nanoparticles of various sizes and morphologies [149] is one of the most promising platforms that can encompass all the aforementioned requirements. Furthermore, they are important biogenic minerals, enabling living organisms to reach specific functions. In nature, vaterites typically form self-assembled polycrystal micro- and nanoparticles. These vaterite particles can tailor alpine plant light scattering channels and utilize the multipole interference effect to improve light collection efficiency by producing $\mathrm{CaCO}_{3}$ polycrystal nanoparticles on the margins of their leaves [150].

Fluorescent microscopy images of the FFF-labeled vaterite microparticles under different excitation wavelengths (Figure 21c-f) demonstrate their ability to serve as multi-color fluorescent agents.

\section{Conclusions}

This review considers a new biophotonic effect of visible fluorescence (FL) observed in all peptide/protein materials folded into $\beta$-sheet secondary structures. TheFL phenomenon has been revealed in fibrillary structures of both natural amyloid fibrils, associated with neurodegenerative diseases (Alzheimer's, Parkinson's, and more), and diverse synthetic peptide/protein structures subjected to thermally induced biological refolding from the helix-like state to a $\beta$-sheet state. We found that, irrespective of the peptide/protein chemical compositions, complexity, and origin, this intrinsic FL effect exhibits a similar broadband tunable spectrum in the range of $400-700 \mathrm{~nm}$, making it a fold-sensitive, optical signature of $\beta$-sheet configurations. Non-covalent hydrogen bonds interconnecting the $\beta$-strands into $\beta$-sheet fibrillary structures are responsible for this highly specific FL phenomenon of biological origin. We also showed that recently developed fluorescent 
hybrid polymer/peptide films, PEG-F6, exhibit quasi-white FL where, in addition to the blue spectral maximum related to the original $\beta$-sheet structure, a sufficient contribution of green FL has been observed. This spectral maximum is associated with a high concentration of amide bonds in this new compound.

We presented a novel type of biolabel, visible FL bionanodots. A completely different approach to the fabrication of these nanodots compared to other biomarker counterparts has been developed. The found method for the inhibition of the self-assembly process of biomolecules allowed us to obtain nanometer-scale peptide/protein biodots which can be considered elementary building blocks of supramolecular biostructures. In the second stage, these dots were transformed from native $\alpha$-helical states to $\beta$-sheet ones by heating at an elevated temperature, which resulted in the appearance of broad band, visible and tunable FL. Single multicolor FL nanodots were visualized. These peptide/protein nanodots are originally biocompatible and can be widely used in nanobiotechnology for medical diagnostics and therapy, the development of a new generation of drugs and safet technology for cosmetics, food products, and more.

In this work, we show that bioinspired nanostructures possess multifunctional optical properties such as nonlinear optical, electrooptical, birefringence, tunable visible fluorescence, and light waveguiding effects. These properties are fold-sensitive and can be functionally switched by the thermally induced refolding of their biological secondary structure from a helix-like state to a $\beta$-sheet state. Such a unique set of optical properties indicates a new paradigm of peptide nanophotonics and the advanced nanotechnology of peptide integrated optics based on recently developed peptide wafers and large-area hybrid polymer/peptide thin films. Biocompatible implantable peptide-integrated optical chips could be applied to light theranostics, optogenetics, and health monitoring.

Author Contributions: Conceptualization, G.R., G.M., B.A., A.A., P.G.; methodology, B.A., A.A., P.G.; theoretical development of the mechanism of long-range FL propagation, B.F.; investigation, B.A., N.L., A.A., C.D., A.H., H.B.; writing—original draft preparation, G.R., B.A., N.L., B.F., G.M., B.A., A.A., C.D., P.G., A.H.; G.R., B.A.; writing-review and editing, G.R., B.A., G.M., P.G. All authors have read and agreed to the published version of the manuscript.

Funding: This research was funded by the Ministry of Science, Technology, and Space of Israel (Grant N79518) and by POR FESR (Italian Regional Operational Program, European Regional Development Fund, 2014/2020 (Project N. B61G18000470007).

Conflicts of Interest: The authors declare no conflict of interest.

\section{References}

1. Chan, F.T.; Kaminski Schierle, G.S.; Kumita, J.R.; Bertoncini, C.W.; Dobson, C.M.; Kaminski, C.F. Protein amyloids develop an intrinsic fluorescence signature during aggregation. Analyst 2013, 138, $2156-2162$. [CrossRef] [PubMed]

2. Handelman, A.; Kuritz, N.; Natan, A.; Rosenman, G. Reconstructive phase transition in ultrashort peptide nanostructures and induced visible fluorescence. Invited feature article. Langmuir 2016, 32, $2847-2862$. [CrossRef] [PubMed]

3. Lapshina, N.; Shishkin, I.; Nandi, R.; Noskov, R.E.; Barhom, H.; Joseph, S.; Apter, B.; Ellenbogen, T.; Natan, A.; Ginzburg, P.; et al. Bioinspired amyloid visible fluorescent nanodots. Adv. Opt. Mater. 2018, 7, 1801400, . [CrossRef]

4. Adler-Abramovich, L.; Aronov, D.; Beker, P.; Yevnin, M.; Stempler, S.; Buzhansky, L.; Rosenman, G.; Gazit, E. Self-assembled arrays of peptide nanotubes by vapour deposition. Nat. Nanotechnol. 2009, 4, 849-861. [CrossRef] [PubMed]

5. Lee, G.-H.; Moon, H.; Kim, H.; Hwang Lee, G.; Kwon, W.; Yoo, S.; Myung, D. Multifunctional materials for implantable and wearable photonic healthcare devices. Nat. Rev. Mater. 2020, 5, 149-165. [CrossRef] [PubMed]

6. Wolfbeis, O.S. An overview of nanoparticles commonly used in fluorescent bioimaging. Chem. Soc. Rev. 2015, 44, 4743-4768. [CrossRef] 
7. Chudakov, D.M.; Matz, M.V.; Lukyanov, S.; Lukyanov, K.A. Fluorescent proteins and their applications in imaging living cells and tissues. Physiol. Rev. 2010, 90, 1103-1163. [CrossRef]

8. Shaner, N.C.; Steinbach, P.A.; Tsien, R.Y. A guide to choosing fluorescent proteins. Nat. Methods 2005, 2, 905-909. [CrossRef]

9. Schnermann, M.J. Organic dyes for deep bioimaging. Nature 2017, 551, 176-177. [CrossRef]

10. Alivisatos, A.P. Semiconductor clusters, nanocrystals, and quantum dots. Science 1996, $271,933-937$. [CrossRef]

11. Mintz, K.J.; Zhou, Y.; Leblanc, R.M. Recent development of carbon quantum dots regarding their optical properties, photoluminescence mechanism, and core structure. Nanoscale 2019, 11, 4634-4652. [CrossRef] [PubMed]

12. Resch-Genger, U.; Grabolle, M.; Cavaliere-Jaricot, S.; Nitschke, R.; Nann, T. Quantum dots versus organic dyes as fluorescent labels. Nat. Methods 2008, 5, 763-775. [CrossRef] [PubMed]

13. Wang, Y.; Hu, A. Carbon quantum dots: Synthesis, properties and applications. J. Mater. Chem. C 2014, 2, 6921-6931. [CrossRef]

14. Ming, N.; Shuangmu, Z.; So, P.T.C.; Yu, H. Fluorescent probes for nanoscopy: Four categories and multiple possibilities. J. Biophotonics 2016, 1, 11-23.

15. Shimomura, O.; Johnson, F.H.; Saiga, Y. Extraction, purification and properties of aequorin, a bioluminescent protein from the luminous hydromedusan, Aequorea. J. Cell Comp. Physiol. 1962, 59, 223-239. [CrossRef] [PubMed]

16. Takuya, T.; Nagano, T. Small-molecule fluorophores and fluorescent probes for bioimaging. Eur. J. Physiol. 2013, 465, 347-359.

17. Lavis, L.D.; Raines, R.T. Bright Ideas for Chemical Biology. ACS Chem. Biol. 2008, 3, 142-155. [CrossRef]

18. Luo, S.; Zhang, E.; Su, Y.; Cheng, T.; Shi, C.A. Review of NIR dyes in cancer targeting and imaging. Biomaterials 2011, 32, 7127-7138. [CrossRef]

19. Weissleder, R.; Ntziachristos, V. Shedding light onto live molecular targets. Nat. Med. 2003, 9, $123-128$. [CrossRef]

20. Cosco, E.D.; Caram, J.R.; Bruns, O.T.; Franke, D.; Day, R.A.; Farr, E.P.; Bawendi, M.G.; Sletten, E.M. Flavylium Polymethine Fluorophores for Near- and Shortwave Infrared Imaging. Angew. Chem. Int. Ed. 2017, 56, 13126-13129. [CrossRef]

21. Ding, F.; Zhan, Y.; Lu, X.; Sun, Y. Recent advances in near-infrared II fluorophores for multifunctional biomedical imaging. Chem. Sci. 2018, 9, 4370-4380. [CrossRef] [PubMed]

22. Donega, C.D.M. Synthesis and properties of colloidal heteronanocrystals. Chem. Soc. Rev. 2011, 40, 1512-1546. [CrossRef] [PubMed]

23. Donegá, C.D.M. Nanoparticles: Workhorses of Nanoscience; Springer: Berlin/Heidelberg, Germany, 2014.

24. Zhao, Y.; van Rooy, I.; Hak, S.; Fay, F.; Tang, J.; Cde, L.D.; Skobe, M.; Fisher, E.A.; Radu, A.; Fayad, Z.A.; et al. Near-Infrared Fluorescence Energy Transfer Imaging of Nanoparticle Accumulation and Dissociation Kinetics in Tumor-Bearing Mice. ACS Nano 2013, 7, 10362-10370. [CrossRef]

25. Wu, Y.; Eisele, K.; Doroshenko, M.; Algara-Siller, G.; Kaiser, U.; Koynov, K.; Weil, T.A. Quantum Dot Photoswitch for DNA Detection, Gene Transfection, and Live-Cell Imaging. Small 2012, 8, 3465-3475. [CrossRef] [PubMed]

26. Liu, H.; Ye, T.; Ma, C. Fluorescent carbon nanoparticles derived from candle soot. J. Mater. Chem. C 2014, 2 , 6921-6939.

27. Sun, Y.-P.; Zhou, B.; Lin, Y.; Wang, W.; Fernando, K.S.; Pathak, P.; Meziani, M.J.; Harruff, B.A.; Wang, X.; Wang, H. Quantum-sized carbon dots for bright and colorful photoluminescence. J. Am. Chem. Soc. 2006, 128, 7756-7757. [CrossRef]

28. Luo, P.G.; Sahu, S.; Yang, S.-T.; Sonkar, S.K.; Wang, J.; Wang, H.; LeCroy, G.E.; Cao, L.; Sun, Y.-P. Carbon "quantum" dots for optical bioimaging. J. Mater. Chem. B 2013, 1, 2116-2127. [CrossRef]

29. Ricciarelli, R.; Fedele, E. The amyloid cascade hypothesis in Alzheimer's disease: It's time to change our mind. Curr. Neuropharmacol. 2017, 15, 926-935. [CrossRef]

30. Sengupta, U.; Nilson, A.N.; Kayeda, N. The role of amyloid- $\beta$ oligomers in toxicity, propagation, and immunotherapy. EBio Med. 2016, 6, 42-49. [CrossRef]

31. Elghetany, M.T.; Saleem, A. Methods for staining amyloid in tissues: A review. Stain Technol. 1988, 63, 201-212. [CrossRef] 
32. Zhao, R.; So, M.; Maat, H.; Ray, N.J.; Arisaka, F.; Goto, Y.; Carver, Y.A.; Hall, D. Measurement of amyloid formation by turbidityassay-seeing through the cloud. Biophys. Rev. 2016, 8, 445-471. [CrossRef] [PubMed]

33. Astolfo, A.; Lathuilière, A.; Laversenne, V.; Schneider, B.; Stampanonia, M. Amyloid- $\beta$ plaque deposition measured using propagation-based X-ray phase contrast CT imaging. J. Synchrotron Radiat. 2016, 23, 813-819. [CrossRef] [PubMed]

34. Streets, A.M.; Sourigues, Y.; Kopito, R.R.; Melki, R.; Quake, S.R. Simultaneous measurement of amyloid fibril formation by dynamic light scattering and fluorescence reveals complex aggregation kinetics. PLoS ONE 2013, 8, e54541, . [CrossRef] [PubMed]

35. Pujol-Pina, R.; Vilaprinyó-Pascual, S.; Mazzucato, R.; Arcella, A.; Vilaseca, M.; Orozco, M.; Carulla, N. SDS-PAGE analysis of A $\beta$ oligomers is disserving research into Alzheimer's disease: Appealing for ESI-IM-MS. Sci Rep. 2015, 5, 14809, . [CrossRef]

36. Mok, Y.F.; Howlett, G.J.; Griffin, M.D. Sedimentation velocity analysis of the size distribution of amyloid oligomers and fibrils. Meth. Enzymol. 2015, 562, 241-256.

37. Randrianjatovo-Gbalou, I.; Marcato-Romain, C.E.; Girbal-Neuhauser, E. Quantification of amyloid fibrils using size exclusion chromatography coupled with online fluorescence and ultraviolet detection. Anal. Biochem. 2015, 488, 19-21. [CrossRef]

38. Zapadka, K.L.; Becher, F.J.; Gomes dos Santos, A.L.; Jackson, S.E. Factors affecting the physical stability (aggregation) of peptide therapeutics. Interface Focus. 2017, 7, 20170030. [CrossRef]

39. Levine, $H$. Thioflavine $t$ interaction with synthetic Alzheimer's disease $\beta$-amyloid peptides: Detection of amyloid aggregation in solution. Protein Sci. 1993, 2, 404-410. [CrossRef]

40. Faisal, A.; Tatulian, S.A. Structure and Aggregation of $A \beta_{1-40}$ and pyroglutamylated $A \beta_{3-40}$ separately and combined. Biophys. J. 2020, 118, 204, . [CrossRef]

41. Diaferia, C.; Balasco, N.; Sibillano, T.; Giannini, C.; Vitagliano, L.; Morelli, G.; Accardo, A. Structural characterization of self-assembled tetra-tryptophan based nanostructures: Variations on a common theme. ChemPhysChem 2018, 19, 1635-1642. [CrossRef]

42. Diaferia, C.; Gosh, M.; Sibillano, T.; Gallo, E.; Stornaiuolo, M.; Gianni, C.; Morelli, G.; Adler-Abramovich, L.; Accardo, A. Fmoc-FF and hexapeptide-based multicomponent hydrogels as scaffold materials. Soft Matter 2019, 15, 487-496. [CrossRef] [PubMed]

43. Khurana, R.; Coleman, C.; Ionescu-Zanetti, C.; Carter, S.A.; Krishna, V.; Grover, R.K.; Roy, R.; Singh, S. Mechanism of thioflavin T binding to amyloid fibrils. J. Struct. Biol. 2005, 151, 229-238. [CrossRef] [PubMed]

44. Donnelly, J.; Vesga, Y.; Hernandez, F.E. Enhanced two-photon absorption and fluorescence upconversion in thioflavin T micelle-type aggregates in glycerol/water solution. Chem. Phys. 2016, 477, 19-23. [CrossRef]

45. Sabate, R.; Lascu, I.; Saupe, S.J. On the binding of Thioflavin-T to HET-s amyloid fibrils assembled at pH 2. J. Struct. Biol. 2008, 162, 387-396. [CrossRef] [PubMed]

46. Nesterov, E.E.; Skoch, J.; Hyman, B.T.; Klunk, W.E.; Bacskai, B.J.; Swager, T.M. In vivo optical imaging of amyloid aggregates in brain: Design of fluorescent markers. Angew. Chem. Int. Ed. 2005, 44, 5452-5456. [CrossRef] [PubMed]

47. Brandenburg, E.; Berlepschz, H.V.; Koksch, B. Specific in situ discrimination of amyloid fibrils versus a-helical fibresby the fluorophore NIAD-4. Mol. Biosyst. 2012, 8, 557-564. [CrossRef]

48. Dyrager, C.; Vieira, R.P.; Nystrom, S.; Nilsson, K.P.R.; Storr, T. Synthesis and evaluation of benzothiazoletriazole and benzothiadiazole-triazole scaffolds as potential molecular probes for amyloid- $\beta$ aggregation. New J. Chem. 2017, 41, 1566-1573. [CrossRef]

49. Yakupova, E.I.; Bobyleva, L.G.; Vikhlyantsev, I.M.; Bobylev, A.G. Congo Red and amyloids: History and relationship. Biosci. Rep. 2019, 39, BSR20181415, . [CrossRef]

50. Diaferia, C.; Gianolio, E.; Palladino, P.; Arena, F.; Boffa, C.; Morelli, G.; Accardo, A. Peptide Materials Obtained by Aggregation of Polyphenylalanine Conjugates as Gadolinium-Based Magnetic Resonance Imaging Contrast Agents. Adv. Funct. Mater. 2015, 25, 7003-7016. [CrossRef]

51. Linke, R.P. Diagnosis of minimal amyloid deposits by congo red fluorescence and amyloid type-specific immunohistochemistry: A review. In Amyloid and Related Disorders; Current Clinical Pathology; Picken, M., Herrera, G., Dogan, A., Eds.; Humana Press: Totowa, NJ, USA, 2015.

52. Crystal, A.S.; Giasson, B.I.; Crowe, A.; Kung, M.-P.; Zhuang, Z.-P.; Trojanowski, J.Q.; Lee, V.M.Y.A. Comparison of amyloid fibrillogenesis using the novel fluorescent compound K114. J. Neurochem. 2003, 86, 1359-1368. [CrossRef] 
53. Klunk, W.E.; Bacskai, B.J.; Mathis, C.A.; Kajdasz, S.T.; McLellan, M.E.; Frosch, M.P.; Debnath, M.L.; Holt, D.P.; Wang, Y.; Hyman, B.T. Imaging A $\beta$ plaques in living transgenic mice with multiphoton microscopy and methoxy-X04, a systemically administered Congo Red derivative. J. Neuropathol. Exp. Neurol. 2002, 61, 797-805. [CrossRef] [PubMed]

54. Schmidt, M.L.; Schuck, T.; Sheridan, S.; Kung, M.P.; Kung, H.; Zhuang, Z.P.; Bergeron, C.; Lamarche, J.S.; Skovronsky, D.; Giasson, B.I.; et al. The fluorescent Congo Red derivative, (trans, Trans)-1-bromo-2,5bis-(3-hydroxycarbonyl-4-hydroxy)styrylbenzene (BSB), labels diverse beta-pleated sheet structures in postmortem human neurodegenerative disease brains. Am. J. Pathol. 2001, 159, 937-943. [CrossRef]

55. Koh, M.; Lee, H.; Lee, Y.; Lee, M. Characterization of early-stage amyloid aggregates by incorporating extrinsic fluorescence and atomic force microscopy. J. Nanosci. Nanotechnol. 2014, 14, 8386-8389. [CrossRef] [PubMed]

56. Diaferia, C.; Balasco, V.; Sibillano, T.; Gogh, M.; Adler-Abramovich, L.; Giannini, C.; Vitagliano, L.; Morelli, G.; Accardo, A. Amyloid-like fibrillary morphology originated by tyrosine-containing aromatic hexapeptides. Chem. Eur. J. 2018, 24, 6804-6817. [CrossRef]

57. Vetri, V.; Canale, C.; Relini, A.; Librizzi, F.; Militello, V.; Gliozzi, A.; Leone, M. Amyloid fibrils formation and amorphous aggregation in concanavalin A. Biophys. Chem. 2007, 125, 184-190. [CrossRef]

58. Reinke, A.A.; Seh, H.Y.; Gestwicki, J.E. A chemical screening approach reveals that indole fluorescence is quenched by pre-fibrillar but not fibrillar amyloid- $\beta$. Bioorg. Med. Chem. Lett. 2009, 19, 4952-4957. [CrossRef]

59. Diaferia, C.; Gianolio, E.; Accardo, A.; Morelli, G. Gadolinium containing telechelic PEG-polymers end-capped by diphenylalanine motives as potential supramolecular MRI contrast agents. J. Pept. Sci. 2017, 23, 122-130. [CrossRef]

60. Younan, N.D.; Viles, J.H. A comparison of three fluorophores for the detection of amyloid fibers and prefibrillar oligomeric assemblies. ThT (thioflavin T); ANS (1-anilinonaphthalene-8-sulfonic acid); and bisANS (4,4'-dianilino- 1,1'-binaphthyl-5,5'-disulfonic acid). Biochemistry 2015, 54, 4297-4306.

61. Hong, Y.; Meng, L.; Chen, S.; Leung, C.W.T.; Da, L.-T.; Faisal, M.; Silva, D.-A.; Liu, J.; Lam, J.W.Y.; Huang, X.; et al. Monitoring and inhibition of insulin fibrillation by a small organic fluorogen with aggregation-induced emission characteristics. J. Am. Chem. Soc. 2012, 134, 1680-1689. [CrossRef]

62. Xue, C.; Lin, T.Y.; Chang, D.; Guo, Z. Thioflavin T as an amyloid dye: Fibril quantification, optimal concentration and effect on aggregation. R. Soc. Open Sci. 2016, 4, 160696, . [CrossRef]

63. D'Amico, M.; Di Carlo, M.G.; Groenning, M.; Militello, V.; Vetri, V.; Leone, M. Thioflavin T promotes A $\beta(1-40)$ amyloid fibrils formation. J. Phys. Chem. Lett. 2012, 3, 1596-1601. [CrossRef] [PubMed]

64. Buell, A.K.; Dobson, C.M.; Knowles, T.P.J.; Welland, M.E. Interactions between amyloidophilic dyes and their relevance to studies of amyloid inhibitors. Biophys. J. 2010, 99, 3492-3497. [CrossRef]

65. Bhasikuttan, A.C.; Mohanty, J. Detection, inhibition and disintegration of amyloid fibrils: The role of optical probes and macrocyclic receptors. Chem. Commun. 2017, 53, 2789-2809. [CrossRef] [PubMed]

66. Kaminski Schierle, G.S.; van de Linde, S.; Erdelyi, M.; Esbjoörner, E.K.; Klein, T.; Rees, E.; Bertoncini, C.W.; Dobson, C.M.; Sauer, M.; Kaminski, C.F. In situ measurements of the formation and morphology of intracellular $\beta$-amyloid fibrils by super-resolution fluorescence imaging. J. Am. Chem. Soc. 2011, 133, 12902-12905. [CrossRef] [PubMed]

67. Cox, H.; Georgiades, P.; Xu, H.; Waigh, T.A.; Lu, J.R. Self-assembly of mesoscopic peptide surfactant fibrils investigated by STORM super-resolution fluorescence microscopy. Biomacromolecules 2017, 18, 3481-3491. [CrossRef] [PubMed]

68. Garai, K.; Frieden, C. Quantitative analysis of the time course of a $\beta$ oligomerization and subsequent growth steps using tetramethylrhodamine-labeled A $\beta$. Proc. Natl. Acad. Sci. USA 2013, 110, 3321-3326. [CrossRef]

69. Hashimoto, T.; Adams, K.W.; Fan, Z.; McLean, P.J.; Hyman, B.T. Characterization of oligomer formation of amyloid- $\beta$ peptide using a split-luciferase complementation assay. J. Biol. Chem. 2011, 286, 27081-27091. [CrossRef]

70. Danzer, K.M.; Ruf, W.P.; Putcha, P.; Joyner, D.; Hashimoto, T.; Glabe, C.; Hyman, B.T.; McLean, P.J. Heat-shock protein 70 modulates toxic extracellular $\alpha$-synuclein oligomers and rescues trans-synaptic toxicity. FASEB J. 2011, 25, 326-336. [CrossRef]

71. Outeiro, T.F.; Putcha, P.; Tetzlaff, J.E.; Spoelgen, R.; Koker, M.; Carvalho, F.; Hyman, B.T.; McLean, P.J. Formation of Toxic Oligomeric $\alpha$-Synuclein Species in Living Cells. PLoS ONE 2008, 3, e1867, . [CrossRef] 
72. Wagele, J.; De Sio, S.; Voigt, B.; Balbach, J.; Ott, M. How fluorescent tags modify oligomer size distributions of the Alzheimer peptide. Biophys. J. 2019, 116, 227-238. [CrossRef]

73. Roberti, M.J.; Bertoncini, C.W.; Klement, R.; Jares-Erijman, E.A.; Jovin, T.M. Fluorescence imaging of amyloid formation in living cells by a functional, tetracysteine-tagged $\alpha$-synuclein. Nat. Methods 2007, 4, 345-351. [CrossRef] [PubMed]

74. Takahashi, T.; Mihara, H. FRET Detection of Amyloid $\beta$ - Peptide oligomerization using a fluorescent protein probe presenting a pseudo-amyloid structure. Chem. Commun. 2012, 48, 1568-1570. [CrossRef] [PubMed]

75. Hatai, J.; Motiei, L.; Margulies, D. Analyzing amyloid beta aggregates with a combinatorial fluorescent molecular sensor. J. Am. Chem. Soc. 2017, 139, 2136-2139. [CrossRef] [PubMed]

76. Liu, H.; Lantz, R.; Cosme, P.; Rivera, N.; Andino, C.; Gonzalez, W.G.; Terentis, A.C.; Wojcikiewicz, E.P.; Oyola, R.; Miksovska, J.; et al. Site-specific dynamics of amyloid formation and fibrillar configuration of aß1-23 using an unnatural amino acid. Chem. Commun. 2015, 51, 7000-7003. [CrossRef]

77. Chiti, F.; Dobson, C.M. Protein misfolding, functional amyloid, and human disease. Annu. Rev. Biochem. 2006, 75, 333-366. [CrossRef] [PubMed]

78. Tuomas, P.J.; Knowles, T.P.J.; Vendruscolo, M.; Dobson, C.M. The amyloid state and its association with protein misfolding diseases. Nat. Rev. 2014, 15, 384-396.

79. Eisenberg, D.; Jucker, M. The amyloid state of proteins in human diseases. Cell 2012, 148, 1188-1203. [CrossRef]

80. Wei, G.; Su, Z.; Reynolds, N.P.; Arosio, P.; Hamley, I.W.; Gazit, E.; Mezzenga, R. Self-assembling peptide and protein amyloids: From structure to tailored function in nanotechnology. Chem. Soc. Rev. 2017, 46, 4661-4692. [CrossRef]

81. Baldwin, A.J.; Knowles, T.P.J.; Tartaglia, G.G.; Fitzpatrick, A.W.; Devlin, G.L.; Shammas, S.L.; Waudby, C.A.; Mossuto, M.F.; Meehan, S.; Gras, S.L.; et al. Metastability of Native Proteins and the Phenomenon of Amyloid Formation. J. Am. Chem. Soc. 2011, 133, 14160-14163. [CrossRef]

82. Shukla, A.; Mukherjee, S.; Sharma, S.; Agrawal, V.; Radha, K.V.; Guptasarma, K.P. A novel UV laser-induced visible blue radiation from protein crystals and aggregates: Scattering artifacts or fluorescence transitions of peptide electrons delocalized through hydrogen bonding? Arch. Biochem. Biophys. 2004, 428, 144-153. [CrossRef]

83. Del Mercato, L.L.; Pompa, P.P.; Maruccio, G.; Della, A.T.; Sabella, S.; Tamburro, A.M.; Cingolani, R.; Rinaldi, R. Charge transport and intrinsic fluorescence in amyloid-like fibrils. Proc. Natl. Acad. Sci. USA 2007, 104, 18019-18024. [CrossRef] [PubMed]

84. Sharpe, S.; Simonetti, K.; Yau, J.; Walsh, P. Solid-State NM characterization of autofluorescent fibrils formed by the elastin-derived peptide GVGVAGVG. Biomacromolecules 2011, 12, 1546-1555. [CrossRef] [PubMed]

85. Kaminski, C.F.; Kaminski-Schierle, G.S. Probing amyloid protein aggregation with optical superresolution methods: From the test tube to models of disease. Neurophotonics 2016, 3, 0418071, . [CrossRef]

86. Shimanovich, U.; Pinotsi, D.; Shimanovich, K.; Yu, N.; Bolisetty, S.; Adamcik, J.; Mezzenga, R.; Charmet, J.; Vollrath, F.; Gazit, E.; et al. Biophotonics of native silk fibrils. Macromol. Biosci. 2018, 18, 1700295, . [CrossRef]

87. Pinotsi, D.; Grisanti, L.; Mahou, P.; Gebauer, R.; Kaminski, C.F.; Hassanali, A.; Kaminski Schierle, G.S. Proton transfer and structure-specific fluorescence in hydrogen bond-rich protein structures. J. Am. Chem. Soc. 2016, 138, 3046-3057. [CrossRef] [PubMed]

88. Pinotsi, D.; Buell, A.K.; Dobson, C.M.; Kaminski Schierle, G.S.; Kaminski, C.F.A. Label-Free, Quantitative Assay of Amyloid Fibril Growth Based on Intrinsic Fluorescence. ChemBioChem 2013, 14, 846-850. [CrossRef] [PubMed]

89. Amdursky, N.; Molotskii, M.; Aronov, D.; Adler-Abramovich, L.; Gazit, E.; Rosenman, G. Blue luminescence based on quantum confinement at peptide nanotubes. Nano Lett. 2009, 9, 3111-3120. [CrossRef]

90. Handelman, A.; Lapshina, N.; Apter, B.; Rosenman, G. Peptide Integrated Optics. Adv. Mater. 2018, 30, 1705776, . [CrossRef]

91. Handelman, A.; Beker, P.; Amdursky, N.; Rosenman, G. Physics and Engineering of peptide supramolecular nanostructures, Perspective Review. Phys. Chem. Chem. Phys. 2012, 14, 6391-6408. [CrossRef]

92. Apter, B.; Lapshina, N.; Handelman, A.; Fainberg, B.; Rosenman, G. Peptide Nanophotonics: From optical waveguiding to precise medicine and implantable biochips. Invited review. Small 2018, 14, 1801147, . [CrossRef] 
93. Lapshina, N.; Jeffet, J.; Rosenman, G.; Ebenstein, Y.; Ellenbogen, T. Single Fluorescent Peptide Nanodots. ACS Photonics 2019, 6, 1626-1631. [CrossRef]

94. Diaferia, C.; Sibillano, T.; Balasco, N.; Giannini, C.; Roviello, V.; Vitagliano, L.; Morelli, G.; Accardo, A. Hierarchical analysis of self-assembled PEGylated hexaphenylalanine photoluminescent nanostructures. Chem. Eur. J. 2016, 22, 16586-16597. [CrossRef] [PubMed]

95. Diaferia, C.; Sibillano, T.; Balasco, N.; Giannini, C.; Roviello, V.; Vitagliano, L.; Morelli, G.; Accardo, A. Structural characterization of PEGylated hexaphenylalanine nanostructures exhibiting green photoluminescence emission. Chem. Eur. J. 2017, 23, 14309-14315. [CrossRef] [PubMed]

96. Johansson, P.K.; Koelsch, P. Label-free imaging of amyloids using their intrinsic linear and nonlinear optical properties. Biomed. Opt. Express 2017, 8, 743-756. [CrossRef]

97. Siddaramaiah, M.; Satyamoorthy, K.; Sadashiva, B.; Rao, S.; Roy, S.; Chandra, S.; Mahato, K.K. Identification of protein secondary structures by laser induced autofluorescence: A study of urea and $\mathrm{GnHCl}$ induced protein denaturation. Spectrochim. Acta Part. A Mol. Biomol. Spectrosc. 2017, 174, 44-53. [CrossRef]

98. Pansieri, J.; Josserand, V.; Lee, S.-J.; Rongier, A.; Imbert, D.; Sallanon, M.M.; Kovari, E.; Dane, T.G.; Vendrely, C.; Chaix-Pluchery, O.; et al. Ultraviolet-visible-near-infrared optical properties of amyloid fibrils shed light on amyloidogenesis. Nat. Photon. 2019, 13, 473-479. [CrossRef]

99. Kholkin, A.; Amdursky, N.; Bdikin, I.; Gazit, E.; Rosenman, G. Strong piezoelectricity in bioinspired peptide nanotubes. ACS Nano 2010, 4, 610-619. [CrossRef]

100. Handelman, A.; Lavrov, S.; Kudryavtsev, A.; Khatchatouriants, A.; Rosenberg, Y.; Mishina, E.; Rosenman, G. Nonlinear optical bioinspired peptide nanostructures. Adv. Opt. Mater. 2013, 1, 875-884. [CrossRef]

101. Gilboa, B.; Lafargue, C.; Handelman, A.; Rosenman, G.; Zyss, J.; Ellenbogen, T. Strong Electro-Optic effect in self assembled peptide structures. Adv. Sci. 2017, 4, 1700052-1700059. [CrossRef]

102. Dobson, C.M. Protein folding and misfolding. Nature 2003, 426, 884-890. [CrossRef]

103. Gorbitz, C.H. Nanotube formation by hydrophobic dipeptides. Chem. Eur. J. 2001, 1, 23-29. [CrossRef]

104. Rosenman, G.; Beker, P.; Koren, I.; Yevnin, M.; Bank-Srour, B.; Mishina, E.; Semin, S. Bioinspired Peptide Nanotubes: Deposition Technology, Basic Physics and Nanotechnology Applications. J. Pept. Sci. 2010, 17, 75-87. [CrossRef] [PubMed]

105. Han, T.H.; Ok, T.; Kim, J.; Shin, D.O.; Ihee, H.; Lee, H.S.; Kim, S.O. Bionanosphere Lithography Via Hierarchical Peptide Self-Assembly of Aromatic Triphenylalanine. Small 2010, 6, 945-951. [CrossRef] [PubMed]

106. Tamamis, P.; Adler-Abramovich, L.; Reches, M.; Marshall, K.; Sikorski, P.; Serpell, L.; Gazit, E.; Archontis, G. Self-Assembly of Phenylalanine Oligopeptides: Insights From Experiments and Simulations. Biophys. J. 2009, 96, 5020-5029. [CrossRef]

107. Amdursky, N.; Beker, P.; Koren, I.; Bank-Srour, B.; Mishina, E.; Semin, S.; Rasing, T.; Rosenberg, Y.; Barkay, Z.; Gazit, E.; et al. Structural Transition in Peptide Nanotubes. Biomacromolecules 2011, 12, 1349-1354. [CrossRef]

108. Englander, S.W.; Mayne, L. The nature of protein folding pathways. Proc. Natl. Acad. Sci. USA 2014, 111, 15873-15880. [CrossRef]

109. Dobson, C.M.; Knowles, T.P.J.; Vendruscolo, M. The amyloid phenomenon and its significance in biology and medicine. Cold Spring Harb. Perspect. Biol. 2020, 12, a033878. [CrossRef]

110. Ranjbar, B.; Gill, P. Circular dichroism techniques: Biomolecular and nanostructural analyses-A review. Chem. Biol. Drug Des. 2009, 74, 101-111. [CrossRef]

111. Stuart, B. Biological Applications of Infrared Spectroscopy; Wiley: Chichester, UK, 1997.

112. Hamley, I.W. Peptide fibrillization. Angew. Chem. Int. Ed. 2007, 46, 8128-8147. [CrossRef]

113. Marshall, K.E.; Serpell, L.C. Structural integrity of $\beta$-sheet assembly. Biochem. Soc. Trans. 2009, 37, 671-676. [CrossRef]

114. Fasman, G.D. Circular Dichroism and the Conformational Analysis of Biomolecules; Plenum Press: New York, NY, USA, 1996.

115. Su, H.R.; Qi, W.; Zhao, J.; He, Z. Hierarchical, interface-induced self-assembly of diphenylalanine: Formation of peptide nanofibers and microvesicles. Nanotechnology 2011, 22, 245609-245613.

116. Woody, R.W. Aromatic side-chain contributions to the far ultraviolet circular dichroism of peptides and proteins. Biopolymers 1978, 17, 1451-1467. [CrossRef]

117. Hamley, Y.W. Peptide nanotubes. Angew. Chem. Int. Ed. 2014, 53, 6866-6881. [CrossRef] [PubMed] 
118. Hauser, C.A.E.; Deng, R.; Mishra, A.; Loo, Y.; Khoe, U.; Zhuang, F.; Cheong, D.W.; Accardo, A.; Sullivan, M.B.; Riekel, C.; et al. Natural tri- to hexapeptides self-assemble in water to amyloid $\beta$-type fiber aggregates by unexpected $\alpha$-helical intermediate structures. Proc. Natl. Acad. Sci. USA 2011, 108, 1362-1365. [CrossRef]

119. Nenhaus, G.U. The green fluorescent protein: A key tool to study chemical processes in living cells. Angew. Chem. Int. Ed. 2008, 47, 8992-8994. [CrossRef]

120. Lakowicz, J.R. Principles of Fluorescence Spectroscopy; Plenum Press: New York, NY, USA, 1983.

121. Amdursky, N.; Gazit, E.; Molotskii, M.; Rosenman, G. Elementary building blocks of self-assembled peptide nanotubes. J. Amer. Chem. Soc. 2010, 132, 15632-15636. [CrossRef]

122. Inagaki, T. Optical absorptions of aliphatic amino acids in the far ultraviolet. Biopolymers 1973, 12, 1353-1362. [CrossRef]

123. Knop, K.; Hoogenboom, R.; Fischer, D.; Schubert, U.S. Poly(ethylene glycol) in drug delivery: Pros and cons as well as potential alternatives. Angew. Chem. Int. Ed. 2010, 49, 6288-6308. [CrossRef]

124. Allen, T.M.; Hansen, C.; Martin, F.; Redemann, C.; Yau-Young, A. Liposomes containing synthetic lipid derivatives of poly(ethylene glycol) show prolonged circulation half-lives in vivo. Biochim. Biophys. Acta 1991, 1066, 29-36. [CrossRef]

125. Fairbanks, B.D.; Schwartz, M.P.; Bowman, C.N.; Anseth, K.S. Photoinitiated polymerization of PEG-diacrylate with lithium phenyl-2,4,6-trimethylbenzoylphosphinate: Polymerization rate and cytocompatibility. Biomaterials 2009, 30, 6702-6707. [CrossRef]

126. Suk, J.S.; Xu, Q.; Kim, N.; Hanes, J.; Ensign, L.M. PEGylation as a strategy for improving nanoparticle-based drug and gene delivery. Adv. Drug Deliv. Rev. 2016, 99, 28-51. [CrossRef] [PubMed]

127. Tanaka, S.; Ogura, A.; Kaneko, T.; Murata, Y.; Akashi, M. Precise synthesis of ABA triblock copolymers comprised of poly(ethylene oxide) and poly( $\beta$-benzyl-l-aspartate): a hierarchical structure inducing excellent elasticity. Macromolecules 2004, 37, 1370-1377. [CrossRef]

128. Smeenk, J.M.; Schon, P.; Mathijs, B.J.; Otten, M.B.J.; Speller, S.; Stunnenberg, H.G.; van Hest, J.C.M. Fibril formation by triblock copolymers of silk-like $\beta$-sheet polypeptides and poly (ethylene glycol). Macromolecules 2006, 39, 2989-2997. [CrossRef]

129. Castelletto, V.; Gouveia, R.J.; Connon, C.J.; Hamley, I.W. Self-assembly and bioactivity of a polymer/peptide conjugate containing the RGD cell adhesion motif and PEG. Eur. Polym. J. 2013, 49, 2961-2967. [CrossRef]

130. Diaferia, C.; Roviello, V.; Morelli, G.; Accardo, A. Self-assembly of PEGylated diphenylalanines into photoluminescent fibrillary aggregates. ChemPhysChem 2019, 20, 2774-2782. [CrossRef]

131. Castelletto, V.; Hamley, I.W. Self-assembly of a model amphiphilic phenylalanine peptide/polyethylene glycol block copolymer in aqueous solution. Biophys. Chem. 2009, 141, 169-174. [CrossRef]

132. Diaferia, C.; Sibillano, T.; Giannini, C.; Roviello, V.; Vitagliano, L.; Morelli, G.; Accardo, A. Photoluminescent peptide-based nanostructures as FRET donor for fluorophore dye. Chem. Eur. J. 2017, 23, 8741-8748. [CrossRef]

133. Apter, B.; Fainberg, B.; Handelman, A.; Lapsker, I.; Accardo, A.; Diaferia, C.; Morelli, G.; Rosenman, G. Long-range fluorescence propagation in amyloidogenic $\beta$-sheet films and fibers. Adv. Opt. Mater. 2020, 8 , 2000056. [CrossRef]

134. Apter, B.; Fainberg, B.; Handelman, A.; Lapsker, I.; Accardo, A.; Diaferia, C.; Morelli, G.; Rosenman, G. Fluorescence waveguiding in amyloidogenic fibers. Proc. SPIE 2020, 11360, 113600D. [CrossRef]

135. Agranovich, V.M.; Galanin, M.D. Electronic Excitation Energy Transfer in Condensed Matter; North-Holland: Amsterdam, The Netherlands; New York, NY, USA, 1983.

136. Agranovich, V.M.; Ratner, A.M.; Salieva, M.K. Excitonic Gratings in Molecular Crystals: Exciton Diffusion, Bimolecular Quenching and Reabsorption of Exciton Fluorescence. Chem. Phys. 1988, 128, 23-33. [CrossRef]

137. Fainberg, B.D. Nonperturbative analytic approach to interaction of intense ultrashort chirped pulses with molecules in solution: Picture of "moving" potentials. J. Chem. Phys. 1998, 109, 4523-4532. [CrossRef]

138. Fainberg, B.D.; Rosanov, N.N.; Veretenov, N.A. Light-induced "plasmonic" properties of organic materials: Surface polaritons, bistability and switching waves. App. Phys. Lett. 2017, 110, 203301-203307. [CrossRef]

139. Amdursky, N.; Gazit, E.; Rosenman, G. Formation of low-dimensional crystalline nucleus regions during insulin amyloidogenesis process. Biochem. Biophys. Res. Commun. 2012, 419, 232-237. [CrossRef]

140. Hoang, H.N.; Abbenante, G.; Hill, T.A.; Ruiz-Gomez, G.; Fairlie, D.P. Folding pentapeptides into left and right handed alpha helices. Tetrahedron 2012, 68, 4513-4516. [CrossRef] 
141. Nakanishi, K.O.; Berova, N.; Woody, R. Circular Dichroism: Principles and Applications; VCH: New York, NY, USA, 1994.

142. Amdursky, N.; Stevens, M.L. Circular Dichroism of Amino Acids: Following the Structural Formation of Phenylalanine. ChemPhysChem 2015, 16, 2768-2774. [CrossRef] [PubMed]

143. Mondai, S.; Adler-Abramovich, L.; Lampel, A.; Bram, Y.; Lipstman, S.; Gazit, E. Formation of functional super-helical assemblies by constrained single heptad repeat. Nat. Commun. 2015, 6, 8615-8623. [CrossRef]

144. Steinfeld, J.I.; Francisco, J.S.; Hase, W.L. Chemical Kinetics and Dynamics, 2nd ed.; Prentice-Hall: Upper Saddle River, NJ, USA, 1999.

145. Datasheet of Tryphenilalanine (FFF) Powder, Bachem (Switzerland). Available online: https://www.bachem. com/service-support/analytical-data-sheet-ads/ (accessed on 27 July 2020).

146. Rust, M.J.; Bates, M.; Zhuang, X. Sub-Diffraction-Limit Imaging by Stochastic Optical Reconstruction Microscopy (STORM). Nat. Methods 2006, 3, 793-795. [CrossRef]

147. Svenskaya, Y.; Parakhonskiy, B.; Haas, A.; Atkin, V.; Lukyanets, E.; Gorin, D.; Antolini, R. Anticancer drug delivery system based on calcium carbonate particles loaded with a photosensitizer. Biophys. Chem. 2013, 182, 1123, . [CrossRef]

148. Parakhonskiy, B.V.; Foss, C.; Carletti, E.; Fedel, M.; Haase, A.; Motta, A.; Migliaresi, C.; Antolini, R. Tailored intracellular delivery via a crystal phase transition in $400 \mathrm{~nm}$ vaterite particles. Biomater. Sci. 2013, 1, 1273-1281. [CrossRef]

149. Bahrom, H.; Goncharenko, A.A.; Fatkhutdinova, L.I.; Peltek, O.O.; Muslimov, A.R.; Koval, Y.O.; Eliseev, I.E.; Manchev, A.; Gorin, D.; Shishkin, I.I.; et al. Controllable synthesis of calcium carbonate with different geometry: Comprehensive analysis of particle formation, cellular uptake, and biocompatibility. ACS Sustain. Chem. Eng. 2019, 7, 19142-19156. [CrossRef]

150. Barhom, H.; Machnev, A.A.; Noskov, R.E.; Goncharenko, A.; Gurvitz, E.A.; Timin, A.S.; Shkoldin, V.A.; Koniakhin, S.V.; Koval, O.Y.; Zyuzin, M.V.; et al. Biological Kerker effect boosts light collection efficiency in plants. Nano Lett. 2019, 19, 7062-7071. [CrossRef] [PubMed]

(C) 2020 by the authors. Licensee MDPI, Basel, Switzerland. This article is an open access article distributed under the terms and conditions of the Creative Commons Attribution (CC BY) license (http://creativecommons.org/licenses/by/4.0/). 